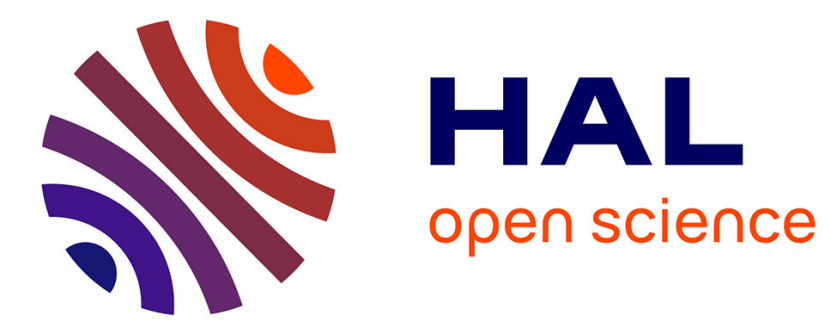

\title{
Un panorama de la recherche française en économie comparant les approches Google Scholar et Econlit
}

Clément Bosquet, Pierre-Philippe Combes

\section{To cite this version:}

Clément Bosquet, Pierre-Philippe Combes. Un panorama de la recherche française en économie comparant les approches Google Scholar et Econlit. 2011. halshs-00652730

HAL Id: halshs-00652730

https://shs.hal.science/halshs-00652730

Preprint submitted on 16 Dec 2011

HAL is a multi-disciplinary open access archive for the deposit and dissemination of scientific research documents, whether they are published or not. The documents may come from teaching and research institutions in France or abroad, or from public or private research centers.
L'archive ouverte pluridisciplinaire HAL, est destinée au dépôt et à la diffusion de documents scientifiques de niveau recherche, publiés ou non, émanant des établissements d'enseignement et de recherche français ou étrangers, des laboratoires publics ou privés. 


\section{GREQAM}

Groupement de Recherche en Economie Quantitative d'Aix-Marseille - UMR-CNRS 6579

Ecole des Hautes études en Sciences Sociales Universités d'Aix-Marseille II et III $n^{\circ} 2011-56$

\section{Document de Travail}

Un panorama de la recherche française en économie comparant les approches Google Scholar et Econlit

Clément Bosquet Pierre-Philippe Combes

December 2011 


\title{
Un panorama de la recherche française en économie comparant les approches Google Scholar et Econlit*
}

\author{
Clément Bosquet $^{\dagger} \quad$ Pierre-Philippe Combes ${ }^{\ddagger}$
}

\section{Décembre 2011}

\begin{abstract}
Résumé
Nous utilisons les citations Google Scholar 2010 des économistes exerçants en France en 2008 afin de dessiner un panorama de la recherche en économie en France et de tester l'utilisation d'un support plus large qu'Econlit pour l'évaluation de la production de recherche. Nous comparons les indicateurs de citations tels que le nombre de citations divisés par le nombre d'auteurs, l'indice $\mathrm{H}$ ou l'indice $\mathrm{G}$ calculés aux niveaux individuels mais aussi des centres de recherche et universités avec des indices de publications calculés avec la base de données EconLit prenant plus ou moins en compte la qualité des journaux. Ces comparaisons sont menées sur différentes périodes de temps.

La hiérarchie des institutions calculée avec l'approche Google Scholar est relativement proche de celle observée lorsque l'on utilise des scores de publications. Néanmoins, on observe également quelques variations spectaculaires pour quelques institutions, positives principalement lorsque l'économie n'est pas le cœur de métier de ces institutions.
\end{abstract}

Codes JEL : I23, A11, R12, $\mathrm{H}_{4}$

Mots clés : économie et géographie de la science, productivité de la recherche, citations, publications

\footnotetext{
* Cet article trouve son origine dans un rapport intitule "Comparaison des mesures Econlit et Google Scholar de la production de recherche en économie en France en 2008" que nous avons produit pour la la Direction Générale de la Recherche et de l'Innovation (DGRI) du Ministère de l'Enseignement Supérieur et de la Recherche. Nous remercions vivement la DGRI pour son soutien financier et Marc Ivaldi pour ses précieux conseils. Nous remercions également Philippe Donnay et Charles Laitong pour leur excellent travail d'assistance de recherche. Les opinions émises dans cet article ne représentent que celles des auteurs et non celles de la DGRI ou du Ministère de l'Enseignement Supérieur et de la Recherche.

${ }^{\dagger}$ Sciences-Po, 28, Rue des Saints-Pères, 75007 Paris, France (clement.bosquet@sciences-po.org ; http :/ / sites.google.com /site/clementbosquet/).

¥Aix-Marseille School of Economics, 2 Rue de la Charité, 13002 Marseille, France (ppcombes@univmed.fr; http ://www.vcharite.univ-mrs.fr/pp/combes/). Egalement affilié au Centre for Economic Policy Research.
} 


\section{Introduction}

Les classements des centres de recherche et universités passent la plupart du temps par une inférence indirecte de la qualité de leurs publications par la qualité du journal dans lequel elles sont publiées. Le principal reproche fait à ce type d'approche est la relativement grande variabilité de qualité qu'il peut rester entre les différents articles d'un même journal. Il est alors souvent proposé d'utiliser directement le nombre de citations reçues par chaque publication. Cela suppose tout d'abord que ce nombre de citations est un bon indicateur de la qualité de la publication, ce qui est généralement admis. Il ne faut pas oublier que cette hypothèse n'est pas complètement triviale, la littérature soulignant par exemple les fortes différences de pratique de citations (nombre de références bibliographiques par article par exemple) entre domaines, et naturellement les différences de nombre de chercheurs de chaque domaine qui affectent directement le nombre de citations susceptibles d'être reçues. La nécessaire pondération des citations reçues par la qualité de la publication citant (comme cela est fait pour évaluer l'impact des journaux par exemple) a priori également nécessaire est souvent difficile à implémenter. Finalement, le nombre de citations d'une publication est très affecté par son cycle de vie, ce qui rend crucial le choix de la fenêtre temporelle pendant laquelle les citations sont recueillies, en fonction de l'âge de la publication. Malgré ces limites, utiliser les citations de chaque publication est souvent considéré comme souhaitable mais rarement effectué du fait du nombre très limité de bases de données le permettant.

La principale source de citations, le Journal of Citation Reports (JCR) de Thomson-Reuters, fournit les citations reçues par les articles publiés dans 304 journaux en économie, alors qu'on en recense plus de 1200 dans la base de données Econlit par exemple (qui regroupe la plupart des revues de recherche en économie). Il est important de noter que la limitation est double : d'une part, une publication n'est citée que si elle appartient à un de ces 304 journaux, d'autre part, les citations ne sont recueillies que dans ces mêmes 304 journaux. Malgré cela, et en ce qui concerne les économistes français, Combes et Linnemer (2003a) comparent pour la France différentes approches qui utilisent les citations JCR afin de hiérarchiser l'impact des publications en économie des centres de recherche et universités françaises en 1998. L'objet du présent article est de déterminer si l'utilisation de Google Scholar (GS) pourrait constituer une alternative intéressante à ces deux stratégies, inférence indirecte de la qualité par celle du journal ou citations du JCR.

GS est un outil qui permet de considérablement dépasser les deux limites que présentent le JCR. GS recense les publications, de tout type, présentes sur des pages Internet académiques et calcule les citations qu'elles reçoivent sur des supports eux-mêmes présents sur Internet, avec la simple condition qu'ils soient également de nature académique. ${ }^{1}$ Ainsi, est élargi à la fois le type de publications susceptibles de recevoir des citations, ce qui prend notamment en compte les supports potentiel-

1. On peut lire sur le site Google Scholar "[Il sagit d'] articles revus par des comités de lecture, thèses, livres, résumés analytiques et articles. Ces travaux peuvent provenir de sources telles que des éditeurs scientifiques, des sociétés savantes, des référentiels de pré-publication, des universités et d'autres organisations de recherche." 
lement importants pour les économistes que constituent les ouvrages ou les documents de travail, mais élargit aussi considérablement le nombre de supports citant. De plus, le domaine d'étude est a priori moins restreint que lorsqu'on le définit ex-ante en le réduisant aux journaux référencées par Econlit ou encore plus par le JCR. Si un économiste a une publication importante dans un journal en mathématiques non référencé par Econlit ou le JCR, celle-ci est en général considérée via GS.

Les limites de GS sont principalement dues au fait qu'il s'agit d'un outil récent, et donc sans aucun doute encore en train de s'améliorer mais sujet à imprécisions, ainsi qu'au fait que GS fonctionne fondamentalement selon la même philosophie que Google, à savoir une recherche de proximité de mots entre diverses entrées, ce qui pose dès le départ la question du seuil à partir duquel on considère que deux groupes de mots sont identiques ou pas. Ainsi, un certain bruit dans la définition de ce qu'est une publication est tout d'abord présent, nous parlerons d'ailleurs souvent d'"entrée" GS plutôt que de publication. Par exemple, différents chapitres d'un ouvrage peuvent constituer autant d'entrées différentes, il en va de même pour un même article publié dans différentes séries de documents de travail, éventuellement à des dates différentes, ou sous des titres légèrement différents, même si un effort certain de regroupement, justement grâce à des algorithmes de proximité, est effectué par GS lui même. Ensuite, on retrouve au niveau des supports citant des sources de bruit de même type. Si deux versions d'un même article considérées comme deux entrées différentes citent un même travail, celui-ci reçoit deux citations. Finalement, les variables relatives à chaque entrée GS sont elles mêmes de qualité nettement moindre que dans Econlit ou JCR. Le nom des co-auteurs est moins précis (absence plus fréquente du prénom complet par exemple), le nombre de co-auteurs est plus difficile à calculer (en partie du fait des imprécisions sur les noms et prénoms), la date de publication n'est pas toujours disponible, ou entachée d'erreur, etc. Cependant, et comme toujours, la seule question importante pour le statisticien est de savoir si les erreurs de mesures sont corrélées ou pas avec le phénomène qu'il tente d'évaluer. Lorsque les erreurs sont distribuées aléatoirement, elles ne gênent en général pas l'analyse.

Malgré ces limites qu'il est crucial de garder en tête, nous proposons ici une étude prospective ayant pour objet de présenter un panorama de la recherche française en économie et d'étudier les propriétés d'un certain nombre d'indicateurs d'impact des centres de recherche et universités françaises en 2008 en économie. Ces indicateurs sont fondés sur les citations GS que reçoivent en janvier 2010 les entrées antérieures à 2008 dans les sujets "Business, Administration, Finance, and Economics" et "Social Sciences, Arts, and Humanities" de leurs membres. Nous calculons, pour tout période de temps $T$ cinq indicateurs de citation GS : le nombre total de citations $(C t(T))$, le nombre de citations par entrée GS $(C e(T))$, le nombre de citations par entrée GS ayant reçu au moins une citation $(C p(T))$, l'indice $H(H(T))$, et l'indice $G(G(T))$, tous ces indices étant dupliqués selon que l'on prend en compte ou pas le nombre de co-auteurs. Cinq périodes de temps différentes sont considérées : toutes les années ( $\mathrm{T}=\mathrm{All}$ ), les cinq dernières années (2004-2008, $\mathrm{T}=5$ ans), en décomptant dans le temps ( $\mathrm{T}=$ Dégressif), par année (d'existence de la publication, $\mathrm{T}=$ Annuel), et par année de 
carrière ( $\mathrm{du}$ chercheur, $\mathrm{T}=$ Carrière). Les résultats obtenus ne sont pas fondamentalement différents entre certaines de ces variantes. Ainsi, avons-nous choisi de nous concentrer dans les sections de classements sur le nombre de citations totales et l'indice $G$, en volume et par chercheur, et sur la période $\mathrm{T}=$ Dégressif. Nous présentons quelques variantes particulièrement intéressantes dans des sections spécifiques et un grand nombre de résultats complémentaires sont donnés dans Bosquet et Combes (2011a). Les résultats obtenus sont systématiquement comparés à ceux utilisant une approche plus traditionnelle basée sur Econlit et la pondération de la qualité moyenne des journaux, de façon modérée $(\mathrm{Clm})$ ou forte $(\mathrm{Clh})$, proposée par Combes et Linnemer (2010).

Les grandes tendances qui se dégagent de notre étude sont les suivantes. Tout d'abord, GS constitue un support de publications entre deux et quatre fois plus large qu'Econlit. Les chercheurs publiant selon Econlit ont plus d'entrées GS que de publications Econlit et la part des non-publiant est plus faible selon GS. Seuls $6.2 \%$ des chercheurs n'ont pas d'entrée GS et $15 \%$ n'en ont pas ayant au moins une citation, alors que $26.6 \%$ n'ont pas de publication Econlit (respectivement, 22.3\%, 34.3\% et 45.3\% sur 2004-2008). L'ensemble des chercheurs localisés en France ont ensemble quasiment 270000 citations GS en janvier 2010, 47000 pour leurs entrées de la période 2004-2008. Chaque entrée a en moyenne presque 7 citations, le double pour celles en ayant au moins une. L'ensemble des entrées GS de la France entraîne un indice H collectif d'environ 180, soit 18o entrées ayant au moins 180 citations et environ 500 entrées ayant en moyenne 500 citations (indice $G$ ).

Prendre ou pas le nombre de co-auteurs ou ramener les citations par entrée ayant ou pas au moins une citation n'a qu'une influence très marginale sur les classements des centres et universités. Il en va de même pour le fait d'utiliser les indices $H$ ou $G$, ou de modifier la prise en compte du temps. Il est important de noter que les approches GS, quelles qu'elles soient, conduisent à une hiérarchie des universités ou centres très proche de celle obtenue via l'approche Clm qui se base sur Econlit. Ainsi, utiliser la qualité des journaux comme prédicteur de la qualité des publications, en tous les cas du nombre de citations GS qu'elles reçoivent, semble constituer une stratégie pertinente, au moins au niveau agrégé des centres et universités.

Notons néanmoins les quelques résultats particuliers suivants. Les indicateurs de citations GS sont moins sélectifs que ne le sont les indicateurs CLh, et se rapprochent plus des indicateurs Clm. Les corrélations entre classements GS et Econlit sont légèrement moins élevées en ce qui concerne la hiérarchie des institutions selon leurs scores par chercheur. La production des chercheurs pour les indices GS, quels qu'ils soient, suit une courbe en cloche au cours de leur cycle de vie, comme on l'obtient pour les indices $\mathrm{Clm}$ et $\mathrm{Clh}$, les chercheurs ayant un pic de productivité généralement observé entre 40 et 50 ans. Les quatre ou cinq statuts les plus productifs selon les indices GS restent identiques à ceux identifiés au moyen de $\mathrm{Clm}$ et $\mathrm{Clh}$ (Ingénieurs Ponts et Chaussées, Directeur d'Etudes Ehess, Administrateurs Insee et Directeurs de recherche Cnrs). En revanche, progressent selon les mesures GS les statuts qui ne sont pas au coeur de notre analyse (hors section 5 Cnu ou section 37 Cnrs, notamment les "assimilés" professeurs ou chargés de recherche, en poste dans des 
grandes écoles, parfois de commerce, ou dans des administrations), l'élargissement du support de publication en étant la cause probable.

En ce qui concerne le classement des universités et centres, il découle de ces résultats que d'une part, pour une majorité de centres ou universités, la variation de classements par rapport à ceux obtenus selon l'indice Econlit Clm est faible. D'autre part, pour quelques autres, elles est relativement forte, et il s'avère que ces unités semblent être celles dont le cœur de l'activité n'est pas l'économie, ou, ce qui est lié, dont les membres ne sont pas au cœur de notre champ, à savoir les sections $5 \mathrm{du}$ Cnu et 37 du Cnrs. C'est cette dernière propriété qui peut faire de GS un instrument complémentaire intéressant de l'instrument Econlit bien que susceptible d'être plus entaché d'erreurs de mesure.

\section{Champ de l'étude et choix méthodologiques}

\subsection{Chercheurs, centres et universités}

Nous considérons 105 centres de recherche français en économie (section 5 du Cnu ou section 37 du Cnrs) d'au moins 5 chercheurs. Ces centres sont soit des Unités Mixtes de Recherche (Université, Grandes Ecoles et Cnrs ou Inra), soit l'ensemble des enseignants-chercheurs d'une université n'appartenant justement pas à de telles Umr. Ces centres s'agrègent en 76 'universités' (qui peut être en fait une Grande Ecole). En moyenne, un centre a 26 chercheurs et une université en rassemble 36, 2832 chercheurs équivalent temps plein étant pris en compte dans l'étude. Le vocable chercheur utilisé ici correspond soit à des enseignants-chercheurs de l'Université ou des Grandes Ecoles, soit à des chercheurs Inra ou Cnrs. Un chercheur peut être affilié à plusieurs centres ou universités, voire être en partie aussi affecté à des universités étrangères, d'où la notion d'équivalent temps (en France). Un système de poids, égalitaire dans la plupart des cas, mais parfois légèrement différent notamment pour les affiliations à l'étranger, donne la clé de répartition du chercheur entre ses différentes affiliations. Les caractéristiques démographiques des chercheurs et leur répartition entre différentes institutions ou status peuvent être trouvées dans Bosquet, Combes, et Linnemer (2010). Rappelons simplement ici qu'environ 90\% des chercheurs n'ont qu'une seule affiliation, mais que les $10 \%$ en ayant plus d'une rassemblent environ $40 \%$ de la production, ce qui souligne l'importance des choix de ventilation de celle-ci entre les différentes affiliations. Par ailleurs, la structure par âge des chercheurs est clairement bi-modale, avec un premier pic aux environs de 38 ans et un deuxième autour de 60 ans.

\subsection{Mesure des stocks de publications via Econlit}

Nous commençons par rappeler les caractéristiques des approches Clm et Clh basées sur Econlit proposées par Bosquet, Combes, et Linnemer (2010) auxquelles nous allons comparer les approches fondées sur GS. Les publications prises alors en compte correspondant à la catégorie "Journal ar- 
ticle" de la version de juin 2009 de la base de données Econlit de l'American Economic Association, dont nous extrayons les publications jusqu'en 2008 inclus. Cette base considère en 2008 plus de 1200 journaux, pour un total de 556770 articles publiés entre 1969 et 2008. Pour chaque article, les informations suivantes sont utilisées : date de publication, journal où il a été publié, nombre de pages, nombre de co-auteurs, codes Jel de classification des domaines. Les mesures de stock de publications d'un chercheur dépendent de deux éléments cruciaux, le système de pondération des journaux dans lesquelles les articles sont publiés, $W$ (pour "Weight"), et le système de pondération des articles en fonction de la période où ils sont publiés, $T$ (pour "Time"). Deux autre dimensions sont également prises en compte, le nombre de co-auteurs ainsi que la longueur de l'article. Ces différents éléments sont combinés de la manière suivante.

Soit $A(i)$ l'ensemble des articles (référencés dans Econlit et donc publiés entre 1969 et 2008 ici) du chercheur $i$. Soit $a$ un article dans $A(i)$. Chaque article est caractérisé par son nombre d'auteurs $n(a)$, son nombre de pages $p(a)$, son année de publication $t(a)$, la longueur moyenne d'un article publié la même année dans le même journal $\bar{p}(a)$ et enfin le poids accordé au journal où il a été publié $w(a)$ appartenant au système de pondération des journaux $W$. Le stock de publications du chercheur $i$ selon $W$ sur la période $T$ est évalué à l'aide de la formule suivante :

$$
y_{i}(T, W)=\sum_{a \in A(i)} T(t(a)) \frac{w(a)}{n(a)} \frac{p(a)}{\bar{p}(a)}
$$

où $T($.$) est la fonction qui pondère les années, détaillée plus bas.$

Au niveau individuel, la manière de prendre en compte le nombre de co-auteurs fait débat. Tout le monde s'accorde sur le fait que publier seul un article représente une plus forte contribution que de le faire à plusieurs. Supposer, comme nous le faisons ici qu'une publication co-écrite à $n$ auteurs compte $n$ fois moins qu'un article publié seul semble à certains trop extrême. Des auteurs (par exemple Lubrano, Bauwens, Kirman, et Protopopescu (2003)) proposent non pas de diviser par $\frac{1}{n}$ mais plutôt par $\frac{1}{\sqrt{n}}$. Cela pose cependant à la fois un problème d'interprétation économique et un problème d'agrégation. Si deux personnes écrivent ensemble un article, $\frac{1}{\sqrt{2}} \approx 0.71$ article est attribué selon cette méthode à chaque auteur. Autrement dit, en joignant deux productions de 0.71 article, on obtient un seul article. Cette hypothèse postule donc implicitement que l'on aurait des rendements d'échelles décroissants dans la production d'articles de recherche. On a alors du mal à comprendre pourquoi autant de gens choisissent de co-publier. De plus, au niveau agrégé d'un centre de recherche par exemple, cette méthode conduit à comptabiliser l'article comme 1.42 article si les deux auteurs sont membres du centre en question alors qu'un seul article est réellement publié par le centre. Enfin, cette comptabilisation donnerait clairement des incitations à ajouter le maximum d'auteurs du centre sur toutes ses publications, ce qui semble d'ailleurs se produire dans certains domaines.

A l'inverse, si l'on pense que les chercheurs travaillent en équipe justement pour bénéficier de 
complémentarités et de rendements d'échelle croissants, on pourrait vouloir utiliser une fonction puissance supérieure à 1 (en valeur absolue). Par exemple, pourrait être attribué à chaque auteur seulement $\frac{1}{n^{1.5}}$ de l'article. Dans le cas de deux auteurs, chacun obtiendrait une contribution de 0.35, soit 0.7 à deux, les 0.3 "manquant" pour obtenir un article représentant l'impact de l'externalité. Mais si l'on pense que cette externalité doit tout de même être attribuée aux auteurs de la publication, et par suite à leur centre ou université, chacun atteint bien une part de $0.35+\frac{0.3}{2}=0.5$, ce qui correspond à notre approche.

Finalement, notons que l'économie se distingue d'autres domaines par le fait que les auteurs ne sont que très rarement hiérarchisés et simplement indiqués par ordre alphabétique. La profession admet donc explicitement que les bénéfices de la publication doivent être équitablement partagés entre auteurs, ce que notre approche choisit également. Avoir des auteurs présentés en ordre non alphabétique soulève des problèmes auxquels il n'existe, autant que nous le sachions, pas de solution faisant consensus. Typiquement s'il est admis que le premier auteur se voit attribuer une fraction de l'article supérieure à l'inverse du nombre d'auteurs, chacun semble avoir un avis personnel sur ce que doit être cette fraction, et cette question se pose pour chaque rang de co-autorat. Le point de vue égalitaire des économistes, quasiment toujours adopté même si quelques exceptions existent, simplifie donc grandement la tâche à ce niveau.

Lorsque tous les articles d'Econlit sont considérés, la répartition est approximativement de $60 \%$ des articles écrits seul, 30\% écrits à deux et 10\% écrits à trois ou plus. Sur les cinq dernières années, le pourcentage d'articles écrits seul diminue pour devenir inférieur à 50\%. Par rapport à ces tendances obtenues sur l'ensemble d'Econlit, les chercheurs français publient moins seuls, n'étant désormais plus qu'environ un tiers à le faire.

Prendre en compte la longueur des articles est un choix naturel. L'article median d'Econlit a 15 pages mais $25 \%$ des articles en ont plus de 21 et $10 \%$ plus de 28 . À l'autre extrême, $25 \%$ des articles ont moins de 9 pages et 10\% moins de 5 . La disparité est importante. On peut penser que le nombre d'idées et innovations dans les articles les plus courts est moins important que dans les plus longs. Toutefois, une question de croissance des rendements émerge là aussi : la dernière page d'un très long article a-t-elle la même importance que celle d'une article très court? Comme pour les co-auteurs, une idée serait de considérer non pas le nombre de pages publiées mais sa racine pour prendre en compte cette décroissance des rendements. Bien que l'argument soit pertinent, le choix de la forme fonctionnelle reste délicat. De plus, un tel choix pose, comme pour les co-auteurs, des problèmes d'agrégation, puisque scinder un article en deux améliorerait le score (même si cette pratique est parfois déjà observée $\mathrm{du}$ fait d'un grand nombre de mesures reposant sur le seul nombre de publications).

Le deuxième problème est celui de la comparaison du nombre de pages entre les différents journaux. D'une part, la typographie et la taille des pages diffèrent entre journaux. Par exemple, une page dans l'American Economic Review contient plus de texte qu'une page dans le Journal of Po- 
litical Economy ou le Quarterly Journal of Economics. D'autre part, les journaux ont des longueurs moyenne d'articles différentes. Cependant, il est crucial de remarquer que les pondérations de la qualité des journaux utilisées s'entendent pour un article moyen du journal. Ainsi, ces pondérations sont censées déjà prendre en compte ces différences de typographie et de longueur moyenne des articles et il n'est donc pas nécessaire de corriger à nouveau cette caractéristique. Typiquement, le score relatif d'Economics Letters et de l'American Economic Review prend en compte le fait que le premier journal publie des articles plus courts. En revanche, parmi les articles d'un même journal, nous supposons qu'un article est plus long uniquement s'il recèle plus d'idées et d'innovations. Pour cette raison, les approches $\mathrm{Clm}$ et $\mathrm{Clh}$ corrigent la mesure de la qualité d'un article par son nombre de pages ramené au nombre de pages moyen, pour l'année de publication, des articles du journal. Ainsi, un article de nombre de pages égal au nombre moyen de pages des articles du journal de la même année a une qualité égale à la qualité du journal. S'il est 20\% plus long, sa qualité est considérée comme $20 \%$ supérieure. Notons que la correction effectuée revient à modifier de moins de $50 \%$ l'indice de qualité du journal pour $80 \%$ des articles et que les français, en ayant tendance à publier des articles légèrement plus longs que la moyenne, sont plutôt avantagés par cette pondération.

Finalement, il existe un très grand nombre de façons de pondérer la qualité des journaux scientifiques. Afin de disposer d'un outil à jour et couvrant l'ensemble des journaux d'Econlit, Combes et Linnemer (2010) font un tour d'horizon des stratégies possibles et construisent plusieurs indices de qualité des journaux. Ceux-ci reposent uniquement sur des moyennes de citations reçues par les journaux, lorsque les indices de citations correspondant existent, soit environ 300 journaux (un quart d'Econlit). Lorsqu'ils n'existent pas, un modèle économétrique prédit quel serait l'indice de citation du journal. Nous retenons in fine deux indices de la qualité des journaux, prenant plus (Clh) ou moins $(\mathrm{Clm})$ en compte les disparités de qualité, telle qu'appréhendée par les citations qu'ils reçoivent, des journaux. De manière importante, la hiérarchie des journaux est la même pour Clm et $\mathrm{Clh}$. La différence entre les deux vient uniquement du degré d'inégalité (convexité) des poids. Les deux systèmes de pondération n'excluent aucun journal recensé par Econlit, tous les journaux ont un poids strictement positif, alors que la plupart des classements de journaux en excluent une grande majorité (typiquement, les indices de citations considèrent au maximum un quart des journaux d'Econlit). Finalement, notons que la qualité du journal est évaluée en 2008 même pour les articles publiés une autre année.

Notons que les chercheurs français publient toujours beaucoup dans des journaux francophones. La Revue Économique est la plus utilisée avec 192 articles pour les chercheurs de notre base (elle compte pour $5 \cdot 7 \%$ du stock $2004-2008$ des articles des chercheurs actifs en 2008). Les chercheurs de notre base ont publiés quasiment 55\% des 354 articles de la Revue Économique sur cette période. Les huit journaux les plus fréquemment utilisés sont tous francophones. Les 15 journaux les plus utilisés cumulent un tiers des publications. Parmi eux, seuls Economics Bulletin et Economics Letters ne sont pas francophones. Parmi les journaux suivants en anglais, on trouve le Journal of Economic 
Theory, le Journal of Public Economics, l'European Economic Review, le Journal of Mathematical Economics et Economic Theory. Ces journaux reflètent une certaine préférence des chercheurs français pour la formalisation. Toutefois, ces cinq journaux représentent ensemble légèrement moins d'articles que ceux de la Revue Économique.

En ce qui concerne la prise en compte du temps, au moins deux approches sont possibles pour décrire les publications d'un centre de recherche. La première, dite de flux, attribue à une institution les publications de l'année $t$ de ses membres de l'année $t$. Cette mesure est éventuellement passée si $t$ ne correspond pas à la date de réalisation de l'étude, ou cumulée, si l'on somme sur plusieurs années. À la date de l'étude, il est possible que certains chercheurs de l'institution n'en soient plus membres, un phénomène d'autant plus important que l'on recule dans le temps ou que l'on somme sur de nombreuses années. Pour un décideur souhaitant allouer un budget à un centre donné ou pour un étudiant souhaitant choisir un lieu où effectuer sa thèse, cette mesure n'est pas forcément la plus pertinente.

La deuxième approche, retenue ici, ne s'intéresse pas tant à la production issue de l'université mais au stock de capital recherche présent à une date donnée. Le stock à la date $t$ consiste en la somme des publications passées (éventuellement en limitant la période de temps) des membres présents dans l'institution à la date $t$, indépendamment de la localisation de ces chercheurs au moment de la publication. Notons que cette approche permet aussi de suivre l'évolution des institutions au court du temps, à partir des effectifs de ces institutions à différentes dates. Pouvoir s'appuyer sur de telles listes, et non sur les affiliations référencées dans Econlit, est également un avantage pour deux raisons. D'une part, la qualité des affiliations déclarées dans Econlit est très mauvaise (en tout cas pour la France), d'autre part, elle ne permet pas d'identifier les chercheurs ne publiant pas, rendant impossible le calcul des scores par chercheur de l'institution. Plus la période est courte, plus les approches flux et stocks se rejoignent, elles seraient mêmes identiques pour la dernière année en l'absence des délais de publications.

Pour revenir au choix de la période de temps proprement dit, de nombreux classements utilisent les publications des cinq dernières années afin de mettre l'accent sur la recherche récente. Ce choix est d'ailleurs prudent lorsque des flux de publications sont mesurés. Dans notre approche en termes de stock, nous notons $\mathrm{T}=5$ ans, ce choix. Nous mesurons aussi un stock fondé sur les publications de toutes les années incluses dans d'Econlit, de 1969 à 2008, ce que nous notons T=All.

Pour une longue période de recensement des publications la question du poids donné à chaque année se pose. Par analogie avec un stock de capital, une publication se déprécie dans le temps. Des publications récentes sont davantage susceptibles d'indiquer que l'institution est à la frontière de la recherche que des publications remontant au début des années soixante-dix. Cette idée est capturée de manière assez brutale lorsque seules les cinq dernières années sont considérées. Une manière plus naturelle de le faire est d'introduire un facteur de dépréciation. Cela donne notre troisième façon d'appréhender l'âge des publications que nous notons T=Dégressif. Nous utilisons un facteur 
d'escompte qui obéit à une fonction logistique $T(t(e))=\frac{1-\exp \left(-10 /(2009-t(e))^{1.8}\right)}{1+\exp \left(-20 /(2009-t(e))^{1.2}\right)}$ dont la valeur pour les 20 premières années est donné dans le Tableau 1. Ainsi, un article de 2008 compte pour 1, un article de 2007 pour 0.943 articles, un de 2006 pour 0.746 etc.

TABLE 1 - Décompte dans le temps pour T=Dégressif

\begin{tabular}{rrrr}
\hline \hline Année & Coef. & Année & Coef. \\
\hline 2008 & 1.000 & 1998 & 0.094 \\
2007 & 0.943 & 1997 & 0.079 \\
2006 & 0.746 & 1996 & 0.067 \\
2005 & 0.549 & 1995 & 0.058 \\
2004 & 0.402 & 1994 & 0.050 \\
2003 & 0.299 & 1993 & 0.044 \\
2002 & 0.227 & 1992 & 0.039 \\
2001 & 0.177 & 1991 & 0.035 \\
2000 & 0.141 & 1990 & 0.031 \\
1999 & 0.114 & 1989 & 0.028 \\
\hline
\end{tabular}

Note $:$ coef. $=$ coefficient de décompte en fonction du temps pour $\mathrm{T}=$ Dégressif.

Enfin, nous considérons une dernière période de temps très différente des autres, prenant en compte l'âge des chercheurs. Elle consiste à diviser le score d'un chercheur par un nombre d'années de carrière, nous la notons $\mathrm{T}=$ Carrière. Dans nos travaux antérieurs, faute d'information relative à l'âge des chercheurs, nous utilisions le nombre d'années depuis la première publication. Cela pouvait poser des problèmes d'interprétation pour les chercheurs publiant sur le tard pour lesquels la période de recherche était considérée comme courte et donc ceux-ci très productifs en moyenne. L'idéal est de considérer l'âge académique, à savoir le temps écoulé depuis le doctorat. Nous ne disposons pas de cette variable et tous les chercheurs ne sont en fait pas titulaires d'un doctorat. Nous utilisons donc ici l'âge des chercheurs moins 26 ans (l'âge minimum dans la base étant 27 ans). Si au niveau individuel ces différentes façons de procéder pour appréhender la durée de carrière du chercheur peuvent créer de grandes différences, nous n'en avons pas constaté au niveau agrégé des centres/universités. Notons finalement que ramener la mesure de publication à ce nombre d'années peut s'effectuer aussi bien pour $\mathrm{T}=5$ ans, $\mathrm{T}=\mathrm{All}$ ou $\mathrm{T}=$ Dégressif. Néanmoins, nous ne l'appliquons ici qu'à $\mathrm{T}=\mathrm{All}$ afin de limiter le nombre de variantes.

Les mesures de publications d'un chercheur donné étant définies, nous pouvons discuter la façon dont celles-ci vont être agrégées par institution. Comme dit plus haut, notre approche nous permet de définir $\alpha_{i}(j)$ la part des publications du chercheur $i$ qui va être affectée à l'institution (centre ou université) $j$. Le stock de publications $Y_{j}(T, W)$ de l'institution $j$ pour un système de pondération $W$ 
des journaux et un système $T$ de pondération des années est donnée par

$$
Y_{j}(T, W)=\sum_{i} \alpha_{i}(j) y_{i}(T, W)
$$

où $y_{i}(T, W)$ est le stock de publications du chercheur $i$ explicité dans l'équation (1) ci-dessus. A ce stock est associé un nombre équivalent de chercheurs temps plein dans l'institution,

$$
N_{j}=\sum_{i} \alpha_{i}(j)
$$

Cela conduit à une mesure de stock de publications par chercheur $\left(Y_{j}^{p c}(T, W)\right)$ de l'institution $j$

$$
Y_{j}^{p c}(T, W)=\frac{Y_{j}(T, W)}{N(j)}
$$

\subsection{Les indices de citations Google Scholar}

L'étape préliminaire nécessaire à notre travail consiste en l'obtention des citations telles que recensées par GS reçues par chaque publication des 2832 chercheurs de notre base. Cela a été effectué via la mise au point d'une routine informatique permettant cette extraction en quelques heures. Ce point est important puisque le nombre de citations GS évolue de jour en jour sans qu'aucun historique ne soit accessible. Nous utilisons ici une extraction effectuée en janvier 2010, soit environ 2 ans après la date à laquelle nous souhaitons mesurer l'impact des centres et universités, ce qui est raisonnable étant donné le temps nécessaire à l'émergence des citations des travaux. ${ }^{2}$

Deux points sont cruciaux. Tout d'abord, l'extraction se fait sur la base du nom de famille et du premier prénom, alors que lorsque nous travaillons sur la base de données Econlit, l'extraction ne porte que sur le nom de famille et l'initiale du prénom sauf dans le cas d'homonymes pour lesquels nous utilisons alors le prénom complet. GS étant beaucoup plus large qu'Econlit, le risque de présence d'homonymes serait largement accru avec une telle stratégie, sans qu'il soit possible d'y faire quoi que ce soit, comme par exemple utiliser les affiliations (déclarées dans Econlit) pour affiner la répartition en cas d'homonymie. A l'inverse, des essais nous ont montré qu'extraire sur la base du prénom complet induit une certaine perte de travaux cités. Entre deux maux, nous avons donc choisi le second, en notant qu'il n'y a pas vraiment de raisons pour que la perte soit plus importante pour certaines institutions que pour d'autres, ce qui est le critère important pour notre étude. Notons que comme pour Econlit, pour les personnes ayant des noms doubles, ou pour les femmes mariées, dont nous connaissons donc deux noms de famille notés nom 1 et nom2, l'extraction est effectuée sur toutes les variantes possibles, à savoir, "nom1, prénom", mais aussi "nom2, prénom", "nom1-nom2,

2. Nous ignorons les entrées GS de 2009, d'une part parce que les données pour 2009 semblent être très incomplètes (à la date où l'extraction a été effectuée, janvier 2010) puisque référençant moitié moins d'entrées que 2008, d'autre part, par souci de comparabilité avec la période $\mathrm{T}=5$ ans utilisée pour les approches Econlit qui s'arrête en décembre 2008. 
prénom" et "nom2-nom1, prénom". Pour les prénoms doubles, seul le premier est conservé, ce qui en l'occurrence ne semble pas vraiment accroître le nombre d'homonymes. Une dernière remarque, plus technique quoique relativement triviale mais importante pour la réplicabilité de notre étude, est que l'extraction est faite en écrivant le critère de recherche entre guillemets afin de ne pas extraire les entrées des personnes de même nom et de prénom différent (ou le contraire).

Le deuxième élément crucial dans l'extraction des données sous GS est que nous restreignons les domaines (option "subject areas" de GS) aux catégories "Business, Administration, Finance, and Economics" et "Social Sciences, Arts, and Humanities". Il aurait été tentant de considérer tous les domaines, et ce pour au moins deux raisons. D'une part, un des intérêts de GS est de prendre en compte les travaux d'économistes en dehors du domaine de l'économie. Cela est partiellement fait ici en considérant ces deux domaines déjà très larges, mais des publications en mathématiques pures par exemple ne sont pas considérées. D'autre part, l'allocation d'une publication à un domaine donné est faite automatiquement par GS, toujours via des algorithmes de proximité de mots clés. Ainsi, une publication dont le titre inclut le mot "gravité" à de fortes chances d'être classée dans le domaine "Physics, Astronomy, and Planetary Science". Les publications en économie sur les flux gravitaires de marchandises s'y retrouve donc en général également affectées. En se restreignant à ces deux domaines, il y a donc également un risque, faible tout de même, de perdre des publications véritablement en économie. Malgré ces remarques, et bien que nous effectuions les extractions sur la base du nom et du prénom des chercheurs, il est cependant nécessaire de restreindre les domaines afin de limiter les problèmes d'homonymie. Quelques essais, qui n'ont pu être systématiques faute de temps et moyens, semblent montrer que cette combinaison des sujets et du critère de recherche donne les résultats les plus fiables sur les économistes. Finalement, notons que notre procédure a été appliquée directement sur GS mais qu'elle donne des résultats quasiment identiques à ceux obtenus grâce au logiciel "Publish or Perish" de Harzing (2007), en utilisant les mêmes critères et domaines de recherche naturellement.

In fine, l'extraction produit, pour chaque chercheur, une observation pour chacune de ses entrées recensées par GS. A chaque entrée est associée son nombre de citations sur un site académique présent sur Internet. Nous ne pouvons malheureusement pas recueillir de façon automatisée l'information relative à la source de chacune de ces citations, c'est-à-dire le détail de chacune des entrées citant. Les noms des co-auteurs, et donc leur nombre, est aussi disponible. Le détail de ceux-ci est limité à 8 auteurs, sachant qu'il a longtemps été limité à 3 pour Econlit. Pour l'économie, cette limite n'est a priori pas très pénalisante puisque $97 \%$ des publications (dans Econlit en tout cas) sont effectuées à trois auteurs ou moins. Finalement, la date de publication est aussi disponible, avec cependant un nombre relativement important de valeurs manquantes, et ce d'autant plus que les dates antérieures à 1969 sont considérées comme aberrantes et transformées en valeur manquantes. 1969 peut paraître un peu récent, ce qui correspond tout de même à quarante années de publications, mais cela permet de disposer de la même date de référence que pour Econlit. Il s'avère aussi qu'un 
nombre relativement conséquent d'entrées est doté d'un nombre nul de citations et qu'il est en fait alors souvent difficile pour ces entrées de déterminer s'il s'agit réellement de ce qu'un chercheur qualifierait de publication. Cela peut être par exemple un résumé dans un programme de colloque, qui, en économie du moins, est rarement cité en l'état. Il s'agit aussi souvent d'erreurs de typographie sur le titre d'autres entrées référencées par ailleurs. Plus loin, certains de nos indices ignorent donc ces entrées sans citations, qui sont également souvent celles sans date.

Le Tableau 2 présente le nombre d'entrées recueillies, total (soit 69460) ou en équivalent écrites seul (42448) selon la méthodologie décrite plus haut c'est à dire en divisant chacune par son nombre d'auteurs. Le tableau souligne aussi le fait que presque exactement la moitié de ces entrées ne reçoit aucune citation. Pour rappel, dans la base de données Econlit, figurent, pour le même ensemble de chercheurs français, 10151 articles équivalent écrits seul. Ainsi, même en ignorant les entrées sans citations et en restreignant les sujets couverts, GS élargit très considérablement l'ensemble des publications considérées en le multipliant par deux voire quatre selon le point de vue (parmi les 10151 articles Econlit, il est fort probable que certains n'ont pas de citations, ne comparer Econlit qu'aux entrées ayant au moins une citation est donc un minimum). Le problème de date de publication manquante est réel (presque 30\% des entrées) mais pas décisif pour que nous ne puissions pas, pour certains indices en tout cas, nous en servir. Notamment, sur les entrées ayant effectivement au moins une citation seulement 10\% d'entre elles n'ont pas de date référencée. Finalement, sur plus courte période ( 5 ans), l'écart en nombre de publications entre GS et Econlit est du même ordre de grandeur.

Comme pour les mesures de publications via Econlit, se pose la question de la prise en considération du temps et d'autres dimensions de la publication, comme son nombre de co-auteurs. Il n'est cependant plus nécessaire de prendre en compte la qualité du support et la longueur des publications, puisque l'hypothèse qui est faite alors est que le nombre de citations reçues capte directement la qualité de chaque entrée.

En ce qui concerne le temps, il est tout d'abord important de souligner que GS ne permet pas de déterminer de façon automatisée quand une citation d'une publication a été effectuée. Ainsi, détenons nous toutes les citations des entrées telles que GS les collecte sur Internet en janvier 2010, sans que l'on sache si ce sont des citations effectuées par une publication ancienne ou récente au delà du fait qu'elle est en ligne en janvier 2010. Cette propriété de GS constitue une limite dans le sens où la littérature utilisant les citations préfère en général accorder plus de poids aux citations récentes afin de mesurer l'importance pour la recherche actuelle de la publication plus que sa valeur passée.

Cela étant dit, il reste possible de jouer sur la date des entrées elles-mêmes, comme pour les mesures Econlit, même si l'on préférerait faire les deux simultanément. Nous considérons donc comme précédemment tout d'abord une fenêtre courte toujours notée $\mathrm{T}=5$ ans. Nous mesurons alors les citations GS telles que référencées en janvier 2010 de toutes les entrées dont la date se situe 
TABle 2 - Caractéristiques des bases utilisées

\begin{tabular}{lcrr}
\hline \hline & & nombre & $\%$ \\
\hline Tout GS & eq. seul & 49460 & 100.0 \\
& & 16106 & 100.0 \\
EconLit & eq. seul & 10151 &. \\
& & 34776 & 50.1 \\
Citation & eq. seul & 20934 & $49 \cdot 3$ \\
& & 49569 & 71.4 \\
Date & eq. seul & 30834 & 72.6 \\
& & 30645 & 44.1 \\
Date et cit. & eq. seul & 18402 & $43 \cdot 4$ \\
& & 18724 & 27.0 \\
5 ans & eq. seul & 10378 & 24.4 \\
& & 33131 & $47 \cdot 7$ \\
10 ans & eq. seul & 19076 & 44.9 \\
& & 10421 & 15.0 \\
5 ans et cit. & eq. seul & 5540 & 13.1 \\
& & 20234 & 29.1 \\
10 ans et cit. & eq. seul & 11324 & 26.7 \\
& & 6188 &. \\
EL. 5 ans & \multirow{2}{*}{ eq. seul } & 3465 &. \\
\hline
\end{tabular}

Note : Nombre d'entrées et pourcentage par rapport au nombre total d'entrées. Citation = entrées avec au moins une citation; Date = entrées avec date renseignée; Date et cit. = entrées avec date renseignée et au moins une citation; 5 ans et 10 ans $=$ entrées avec date renseignée, dans les 5 et 10 dernières années respectivement; 5 ans et cit. et 10 ans et cit. = entrées avec au moins une citation et date renseignée, dans les 5 et 10 dernières années respectivement.

entre 2004 et 2008. Si $e$ est une entrée GS de date de publication $t(e)$, on peut alors définir comme précédemment une fonction de pondération des années, $T($.$) , telle que T(t(e))=1$ lorsque $t(e) \geq$ 2004 et $T(t(e))=0$ pour les années précédentes ainsi que pour 2009. Le Tableau 2 montre que cette période considère environ un tiers des entrées GS (ligne $\mathrm{T}=5 \mathrm{ans}$ ). L'influence de plus long terme fondée sur toutes les entrées GS est aussi considérée, soit T=All. Dans ce cas, $T(t(e))=1$ pour toutes les dates de publications, y compris quand celles-ci sont manquantes (en excluant toujours 2009). Finalement, comme pour les mesures Econlit, nous pouvons aussi décompter progressivement les publications dans le temps, ce que nous notons T=Dégressif, à l'aide de la même fonction logistique présentée dans le Tableau 1 . Notons qu'il s'agit moins de dire que des citations anciennes ont moins de valeur que de donner moins de poids à la production ancienne. Pour donner moins de poids aux citations anciennes, il faudrait disposer de la date à laquelle les citations sont effectuées, ce que nous n'avons pas. Ce que nous faisons revient à donner moins de poids à une entrée qui a 10 ans et a 
reçu 30 citations qu'à une autre qui aurait reçu également 30 citations mais qui n'aurait que 5 ans. On pourrait souhaiter, en plus, donner un poids différents en fonction de la date de citation. Si les 30 citations du premier article ont été effectuées plus récemment, bien que l'article soit plus ancien, cela lui redonne de la valeur. La contrainte de données nous oblige à donner le même poids à toutes les citations.

Notons que la littérature produit aussi des indices de citations reçues par année, ce que nous notons $\mathrm{T}=$ Annuel. Cela revient en fait à appliquer un taux de décompte dans le temps relativement extrême car proportionnel aux nombre d'années depuis la publication, $T(t(e))=\frac{1}{2009-t(e)}$. Il vaut mieux lire ce chiffre comme un score moyen par an sur une période plus ou moins longue, même si nous ne l'appliquons ici qu'à l'ensemble des entrées GS à date non manquante et comprise entre 1969 et 2008 (et non à celles sur les 5 dernières années par exemple).

Finalement, nous produisons comme pour les approches Econlit des scores "par année de carrière" avec alors $T(t(e))=\frac{1}{\text { âge chercheur moins } 26 \text { ans }}$, indépendante de $t(e)$ donc.

Nous recueillons donc pour chaque chercheur le nombre de citations reçues par chacune de ses entrées GS. Comme pour le décompte simple du nombre de publications, se pose la question de savoir comment cette production est ventilée entre ses différents auteurs. Comme pour les scores Econlit, nous effectuons une ventilation entre auteurs proportionnelle à leur nombre. Si une entrée à trois auteurs recueille 21 citations, chaque auteur se voit attribué un tiers de publication. Des exemples de calculs pour les indices plus sophistiqués de citations, comme les indices H ou G, sont donnés plus bas car ils nécessitent de préciser cette ventilation.

Nous présentons dans les Tableaux 5 et 6 les corrélations entre classements de centres effectuant ou pas cette correction. On trouvera dans Bosquet et Combes (2011a) ces corrélations pour les universités et pour les chercheurs. Les différences sont significatives mais pas majeures. En l'absence d'arguments en faveur de la non-prise en compte totale du nombre d'auteurs, nous préférons donc effectuer cette correction tout au long de cette étude afin également de ne pas multiplier les variantes, déjà nombreuses. A fin de comparaison avec Econlit, le Tableau 3 donne le pourcentage d'entrées GS à un, deux, trois ou quatre auteurs et plus. Les parts de publications à 1 ou 3 auteurs sont remarquablement similaires entre GS et Econlit. Celles à deux auteurs sont relativement moins nombreuses dans GS, alors que celle à plus de trois auteurs le sont plus. Ce dernier résultat s'explique certainement par le fait qu'Econlit n'a longtemps pas recensé le nombre d'auteurs au delà de trois. Dans tous les cas, la proximité de ces distributions est relativement rassurante puisque par ailleurs le nombre d'entrées est bien plus important pour GS : cela ne semble pas engendrer de distorsion très notable sur le co-autorat, sans toutefois que l'on puisse déterminer si les petites différences apparaissant proviennent de la nature des travaux différente entre les deux sources ou d'une pure erreur de mesure liée à la façon dont GS extrait les auteurs des publications.

Le premier indice que nous calculons, noté $C t_{i}(T)$ est le nombre total de citations reçues par les travaux du chercheur $i$, en équivalent reçu seul, travaux produits sur la période de temps $T$ et 
TABLE 3 - Nombre d'auteurs par entrée

\begin{tabular}{cccccc}
\hline \hline Nb. d'auteurs & 1 & 2 & 3 & 4 et + & Total \\
\hline \% GS T=All & 41.5 & 33.6 & 15.0 & 9.9 & 100.0 \\
$\%$ GS T=5 ans & 32.4 & 36.2 & 19.8 & 11.6 & 100.0 \\
\hline$\%$ EconLit T=All & 42.4 & 39.8 & 15.3 & 2.5 & 100.0 \\
$\%$ EconLit T=5 ans & 31.6 & 43.1 & 20.0 & 5.3 & 100.0 \\
\hline
\end{tabular}

référencés par GS en janvier 2010. $E(i)$ est l'ensemble des entrées GS du chercheur i, e une entrée particulière dans cet ensemble, $c(e)$ le nombre de citations qu'elle a reçues, $n(e)$ son nombre de co-auteurs et $t(e)$ sa date de publication. $C t_{i}(T)$ est donné par

$$
C t_{i}(T)=\sum_{e \in E(i)} T(t(e)) \frac{c(e)}{n(e)} .
$$

Un même nombre de citations peut cependant être obtenu soit via un petit nombre de publications très fortement citées, soit par un plus grand nombre de publications moins citées. La profession attribue en général plus de mérite à la première situation, avec sans doute plus ou moins consciemment en tête des exemples de chercheurs ayant une influence considérable via un très petit nombre d'articles publiés. La situation extrême de chercheurs n'ayant eu qu'une publication de grand impact n'est cependant pas toujours considérée comme un idéal, un bon équilibre entre volume total de citations et qualité minimale de chaque contribution étant souvent perçu comme souhaitable. Quoiqu'il en soit, ramener le nombre de citations au nombre d'entrées donne un point de vue de l'influence des travaux d'un chercheur complémentaire de celui du nombre total de citations reçues par ses entrées. Un problème lié à l'utilisation de GS est que, comme dit plus haut, le nombre d'entrées pour un chercheur donné est une variable relativement bruitée du fait de la présence d'un grand nombre d'entrées, en général non citées, difficilement assimilables à ce que l'on appellerait une publication. Afin de calculer la sensibilité de nos mesures à cette imprécision, nous calculons deux indices de nombre de citations reçues par entrée. Le premier, $C e_{i}(T)$, est le nombre de citations reçues sur la période $T$ par entrée du chercheur, quelque soit le nombre de citations reçues par ces entrées, soit $C t_{i}(T)$ divisé par le nombre total d'entrées donc. Le deuxième, $C p_{i}(T)$, est le nombre de citations reçues par entrée ayant reçue au moins une citation. Autrement dit, une entrée n'ayant pas reçue de citation n'est alors pas considérée comme une publication, ce qui n'affecte pas le numérateur mais modifie le dénominateur.

Dans les deux cas, le nombre qui apparaît au dénominateur est bien le nombre d'entrées équivalent écrit seul, puisque la ventilation des citations entre auteurs ne consiste pas à supposer que chaque auteur a écrit un article lui procurant un $1 / n(e)$ des citations, mais bien un $1 / n(e) \mathrm{d}^{\prime}$ article ayant des citations, procurant donc à cet auteur $1 / n(e)$ citations. Notons aussi que la fonction de décompte dans le temps est également prise en compte. Si l'on se restreint aux cinq dernières 
années, il ne faut diviser que par le nombre d'articles publiés sur ces cinq ans et l'on fait naturellement de même avec le taux de décompte continu correspondant à T=Dégressif. En revanche, pour $\mathrm{T}=$ Annuel, la littérature préfère diviser par le nombre total d'articles pour produire un nombre de citations par an et par article pour l'ensemble de la production, ce que nous ferons donc aussi (même si restreindre la période aurait aussi du sens). In fine,

$$
\begin{gathered}
C e_{i}(T)=\frac{C t_{i}(T)}{\sum_{e \in E(i)} \frac{T(t(e))}{n(e)}}, \\
C p_{i}(T)=\frac{C t_{i}(T)}{\sum_{e \in E(i), c(e)>0} \frac{T(t(e))}{n(e)}},
\end{gathered}
$$

sauf pour T=Annuel, $T(t(e))$ étant alors remplacé par 1 au dénominateur.

Du fait, d'une part, de la relative sensibilité des mesures de citations par entrée à l'erreur de mesure sur le nombre d'entrées et du débat sur le fait de compter ou pas celles n'ayant pas de citations, d'autre part, de la nécessité de calculer deux indices, un en volume total et un par publication, pour évaluer l'influence d'un chercheur ou d'une institution, des travaux ont essayé de proposer des indices plus synthétiques combinant ces différentes dimensions. C'est par exemple le cas du désormais fameux indice $H$, proposé par Hirsh (2005). Notre indice $H_{i}(T)$ pour le chercheur $i$ prend la valeur $h$, qui est unique pour ce chercheur, si $h$ de ses publications de la période $T$ ont reçu au moins $h$ citations chacune. L'indice $H_{i}(T)$ est croissant, au sens large, à la fois avec le nombre de publications et le nombre de citations reçues par celles-ci, comme une mesure de volume total telle que $C t_{i}(T)$. Cependant, en ignorant les publications les moins citées, il donne une idée du degré moyen de qualité des publications les meilleures tout en éliminant une partie du bruit provenant de la façon dont on définit une publication.

Un défaut de l'indice $\mathrm{H}$ a cependant été rapidement souligné. Deux chercheurs ou institutions peuvent avoir le même indice alors que l'un d'entre eux a quelques publications nettement plus citées que l'autre. Autrement dit, l'indice $\mathrm{H}$ ignore la distribution interne des citations reçues par les articles contribuant effectivement à son calcul. C'est principalement afin de palier ce problème que l'indice $G$ a été proposé par Egghe (2006). Le chercheur $i$ a un indice $G_{i}(T)$ qui prend la valeur $g$, qui est également unique, si ses $g$ articles de la période $T$ les plus cités ont reçu, ensemble, $g^{2}$ citations, soit $g$ citations en moyenne cette fois-ci. On peut montrer que pour un chercheur donné $G_{i}(T)$ est nécessairement supérieur à $H_{i}(T)$, la différence entre les deux étant liée au nombre de citations reçues en moyenne par les articles les plus cités. Pour deux chercheurs ayant le même $H$, celui dont la moyenne des citations des articles les plus cités est la plus importante a un indice $G$ plus élevé. Alors que ces deux indices ignorent le bas de la distribution des citations, l'indice G présente l'avantage de mieux prendre en compte le haut de celle-ci.

A partir des définitions des indices $\mathrm{H}$ et $\mathrm{G}$ de base, il faut ensuite prendre en compte le nombre 
de co-auteurs. Il suffit pour cela, comme l'a proposé Schreiber (2008) et dans l'esprit de ce qui est fait pour les autres indices, de simplement bien attribuer la totalité des citations à l'article mais simplement une fraction de l'article à chaque auteur. Désormais, l'indice $\mathrm{H}$ n'est plus nécessairement entier mais il garde la même signification. Par exemple, un indice H de 7.5 signifie que l'auteur a publié au moins 7.5 articles équivalent écrit seuls ayant au moins 7.5 citations chacun, c'est-à-dire au moins 8 .

Si la prise en compte du temps affecte la valeur des citations, comme par exemple pour T=Dégressif, l'indice $\mathrm{H}$ peut également être étendu. Il suffit pour cela de décompter le nombre de citations avant d'ordonner les articles par ordre décroissant de celles-ci. Finalement, on peut selon les mêmes principes calculer des indices $\mathrm{G}$ prenant en compte le nombre de co-auteurs et le décompte dans le temps des citations. Le Tableau 4 donne un exemple de ces calculs à partir d'un ensemble fictif de 11 entrées d'un chercheur ayant ou pas reçues des citations GS, dont nous calculons certains de nos indices pour les trois périodes de temps $\mathrm{T}=\mathrm{All}, \mathrm{T}=5$ ans et $\mathrm{T}=$ Dégressif. La première entrée a reçu 10 citations (colonne "cit."), n'a qu'un auteur (colonne "aut.") et a été publiée en 2006 (colonne "date"), et de même pour les suivantes. En fonction de la date de publication, on obtient le poids lié à la période de publication, donné dans la colonne "pp". Celui-ci vaut toujours 1 pour T=All, 1 pour $\mathrm{T}=5$ ans lorsque l'entrée a moins de 5 ans, o sinon, et les poids correspondant à chaque année donnés dans le Tableau 1 pour T=Dégressif. La colonne "pc" donne le poids lié au nombre de coauteurs. La colonne suivante " $c p$ " agrège ces deux informations pour obtenir le nombre pondéré de citations GS qui contribue au score GS obtenu. La colonne "rp" donne finalement le rang, également pondéré, de l'entrée GS, qui est nécessaire pour calculer les indices H et G. Les cinq dernières lignes du tableau donnent les cinq scores GS principaux correspondant à cet exemple, pour chaque de ces trois périodes, que l'on peut retrouver à l'aide des formules données précédemment.

En résumé, nous calculons, sur cinq périodes de temps (toutes les années, $\mathrm{T}=\mathrm{All}$ ), les cinq dernières années (2004-2008, $T=5$ ans), en décomptant dans le temps ( $T=$ Dégressif), par année (d'existence de la publication, $\mathrm{T}=$ =Annuel), et par année de carrière ( $\mathrm{du}$ chercheur, $\mathrm{T}=\mathrm{Carrière),} \mathrm{cinq} \mathrm{indica-}$ teurs de citation GS au niveau individuel : le nombre total de citations $(C t(T))$, le nombre de citations par entrée GS $(C e(T))$, le nombre de citations par entrée GS ayant reçu au moins une citation $(C p(T))$, l'indice $H(H(T))$, et l'indice $G(G(T))$, toutes prenant en compte le nombre de co-auteurs de façon proportionnelle. Naturellement, d'autres variantes sont possibles et ont d'ailleurs fait l'objet de calcul de notre part, comme le fait de ne pas prendre en compte le nombre de co-auteurs. Néanmoins, les résultats obtenus ne sont alors pas fondamentalement différents alors que la présentation en est considérablement alourdie. Ainsi, avons-nous choisi de nous concentrer sur ce groupe d'indices GS, et même, dans les sections de classements, suite aux résultats obtenus quant à la très forte corrélation des classements observées entre certains d'entre eux, sur un sous ensemble de ceux-ci permettant de mettre en valeur différentes dimensions de l'influence des publications des chercheurs.

Maintenant que nous avons défini les mesures de citations d'un chercheur donné, nous pouvons 
TABLE 4 - Exemples de calculs de scores GS

\begin{tabular}{|c|c|c|c|c|c|c|c|c|c|c|c|c|c|c|c|}
\hline \multirow[t]{2}{*}{ entrée } & \multirow[t]{2}{*}{ cit. } & \multirow{2}{*}{\multicolumn{2}{|c|}{ aut. date }} & \multicolumn{4}{|c|}{$\mathrm{T}=\mathrm{All}$} & \multicolumn{4}{|c|}{$\mathrm{T}=5$ ans } & \multicolumn{4}{|c|}{$\mathrm{T}=$ Dégressif } \\
\hline & & & & $\mathrm{pp}$ & pc & $\mathrm{cp}$ & $\mathrm{rp}$ & $\mathrm{pp}$ & $\mathrm{pc}$ & $\mathrm{cp}$ & $\mathrm{rp}$ & $\mathrm{pp}$ & $\mathrm{pc}$ & $\mathrm{cp}$ & $\mathrm{rp}$ \\
\hline 1 & 10 & 1 & 2006 & 1 & 1 & 10 & 1 & 1 & 1 & 10 & 1 & 0,75 & 1 & 7,46 & 0,75 \\
\hline 2 & 5 & 1 & 2008 & 1 & 1 & 5 & 2 & 1 & 1 & 5 & 2 & $1, \mathrm{OO}$ & 1 & 5,00 & 1,75 \\
\hline 3 & 5 & 1 & 2003 & 1 & 1 & 5 & 3 & $\mathrm{O}$ & 1 & $\mathrm{O}$ & 2 & 0,30 & 1 & 1,49 & 5,81 \\
\hline 4 & 5 & 2 & 2007 & 1 & 0,5 & 5 & 3,5 & 1 & 0,5 & 5 & 2,5 & 0,94 & 0,5 & 4,72 & 2,22 \\
\hline 5 & 3 & 1 & 2008 & 1 & 1 & 3 & 4,5 & 1 & 1 & 3 & 3,5 & $1, \mathrm{OO}$ & 1 & $3, \mathrm{OO}$ & 3,22 \\
\hline 6 & 3 & 1 & 2006 & 1 & 1 & 3 & 5,5 & 1 & 1 & 3 & 4,5 & 0,75 & 1 & 2,24 & 3,96 \\
\hline 7 & 3 & 1 & 2005 & 1 & 1 & 3 & 6,5 & 1 & 1 & 3 & 5,5 & 0,55 & 1 & 1,65 & $5,5^{1}$ \\
\hline 8 & 2 & 1 & 2003 & 1 & 1 & 2 & 7,5 & $\mathrm{O}$ & 1 & $\mathrm{O}$ & 5,5 & 0,30 & 1 & 0,60 & 6,11 \\
\hline 9 & 2 & 1 & 2008 & 1 & 1 & 2 & 8,5 & 1 & 1 & 2 & 6,5 & 1,00 & 1 & $2, \mathrm{OO}$ & 4,96 \\
\hline 10 & 1 & 1 & 2003 & 1 & $I$ & $I$ & 9,5 & $\mathrm{O}$ & 1 & $\mathrm{O}$ & 6,5 & 0,30 & 1 & 0,30 & 6,41 \\
\hline 11 & $\mathrm{O}$ & 1 & 2008 & 1 & 1 & $\mathrm{O}$ & 10,5 & 1 & 1 & $\mathrm{O}$ & 7,5 & 1,00 & 1 & 0,00 & 7,41 \\
\hline \multicolumn{4}{|c|}{$\mathrm{Ct}$} & \multicolumn{4}{|c|}{39.0} & \multicolumn{4}{|c|}{31.0} & \multicolumn{4}{|c|}{28.5} \\
\hline \multicolumn{4}{|c|}{$\mathrm{Ce}$} & \multicolumn{4}{|c|}{$3 \cdot 5$} & \multicolumn{4}{|c|}{$3 \cdot 9$} & \multicolumn{4}{|c|}{3.6} \\
\hline \multicolumn{4}{|c|}{$\mathrm{Cp}$} & \multicolumn{4}{|c|}{3.9} & \multicolumn{4}{|c|}{$4 \cdot 4$} & \multicolumn{4}{|c|}{4.1} \\
\hline \multicolumn{4}{|c|}{$\mathrm{H}$} & \multicolumn{4}{|c|}{$3 \cdot 5$} & \multicolumn{4}{|c|}{2.5} & \multicolumn{4}{|c|}{2.2} \\
\hline \multicolumn{4}{|c|}{ G } & \multicolumn{4}{|c|}{$5 \cdot 5$} & \multicolumn{4}{|c|}{4.5} & \multicolumn{4}{|c|}{4.0} \\
\hline
\end{tabular}

Notes : entrée $=$ numéro entrée GS ; cit. = nombre de citations reçues ; date $=$ date de l'entrée, aut.= nombre de coauteurs ; $\mathrm{pp}=$ poids période $; \mathrm{pc}=$ poids co-auteurs, $\mathrm{cp}=$ citations pondérées ; re= rang de l'entrée.

discuter la façon dont celles-ci sont agrégées par institution. Nous le faisons de la même façon que pour les approches Econlit. : toutes les citations reçues par un chercheur sont affectées au centre de recherche ou à l'université dont il est membre en 2008 et pour les chercheurs à affiliations multiples, une fraction de chaque article est attribué à chaque institution. Ainsi, lorsqu'un chercheur équitablement réparti entre deux centres a une entrée GS ayant reçu 20 citations, le centre est considéré comme ayant obtenu une demi entrée GS ayant reçu 20 citations. La première étape consiste ainsi à établir la liste des entrées GS de chaque centre ou université avec son nombre de co-auteurs et la part qui lui en revient, prenant à la fois en compte la part de co-auteurs effectivement membre du centre et le possible décompte dans le temps. La stratégie de calcul d'indice appliquée aux chercheurs peut alors être appliquée exactement de la même façon aux centres ou universités.

Finalement, on peut vouloir comparer la productivité moyenne des chercheurs entre universités. Pour les indices, $C t(T), C e(T), C p(T)$, il suffit de les diviser par le nombre de chercheurs équivalent temps plein de l'institution. En revanche, pour les indices H et G, il faut bien en faire la moyenne entre chercheurs, pondérée par la part du chercheur dans le centre.

Nous avons déjà mentionné un certain nombre de limites dues à l'imperfection de l'outil GS, qui il est vrai, reste un outil relativement jeune qui devrait s'améliorer avec les années. Certaines entrées ne devraient pas apparaître, d'autres devraient être regroupées, la date de publication de certaines est manquante, le nombre d'auteurs n'est pas toujours facile à établir. Plus important est le 
fait de devoir réduire quelque peu les champs couverts afin de ne pas trop multiplier les problèmes d'homonymes. Ne pas connaître la date à laquelle les citations sont reçues empêche d'aborder une autre famille d'indices prenant en compte la date à laquelle les citations ont eu lieu. Finalement, de façon plus large, connaître entièrement l'origine de la citation permettrait d'affiner les indices produits en ignorant les citations émanant des auteurs eux-mêmes par exemple, ou en pondérant plus fortement les citations provenant d'auteurs, ou de journaux, eux-même fortement cités. La structure actuelle du site internet GS ne permet pas pour le moment d'effectuer de telles variantes.

\subsection{Corrélations entre classements}

Une des conclusions de nos travaux précédents (Combes et Linnemer (2001), Combes et Linnemer (2003b), Bosquet, Combes, et Linnemer (2010)) est la relative insensibilité de la hiérarchie des centres ou universités aux hypothèses effectuées quant à la façon de prendre en compte le nombre de co-auteurs, la longueur des publications, voire la qualité des journaux. Elle est confirmée par la présente étude pour l'approche GS comme le montre les Tableaux 5 et 6.

Prendre ou pas le nombre de co-auteurs en compte n'induit quasiment pas de changement dans la hiérarchie des institutions, la corrélation la plus faible entre deux indices identiques prenant ou pas en compte ce nombre étant par exemple de 0.96 pour l'indice $\mathrm{H}$ au niveau des centres. Pour cette raison, nous préférons utiliser les indices qui prennent en compte ce nombre, puisque, comme expliqué plus haut, l'intuition, en termes d'hypothèses sous-jacentes et d'agrégation, est plus claire.

Pour le nombre de citations reçues par entrée, le fait d'exclure ou pas celles n'ayant aucune citation est lui aussi tout à fait insensible, avec des corrélations supérieures à 0.97 au niveau des classements des centres, entre indices qui le font ou pas, bien que la part d'entrées n'ayant pas de citation soit relativement élevée : le phénomène semble affecter tous les centres dans des proportions relativement similaires. Ces conclusions sur le type d'entrées considérées et la prise en compte du nombre de co-auteurs au niveau des centres se retrouvent non seulement au niveau des universités mais également des chercheurs, comme cela apparaît dans Bosquet et Combes (2011a).

A façons de prendre en compte le nombre d'auteurs et le nombre d'entrées identiques, les corrélations sont élevées entre tous les indices fondés sur les citations GS. Il est intéressant de noter que les corrélations des indices $\mathrm{H}$ et $\mathrm{G}$ sont plus élevées avec les indices de citations totales qu'avec les indices de citations par entrée, ces dernières étant même plus faibles qu'entre les indices en volume total et par entrée. Ainsi, si l'intérêt des indices $\mathrm{H}$ et $\mathrm{G}$ est de prendre en compte la distribution des citations entre publications, ils restent néanmoins fortement corrélés au volume total de citations reçues et beaucoup moins au nombre de citations par entrée. Cela n'est pas tellement plus marqué pour l'indice $\mathrm{H}$ que pour l'indice $\mathrm{G}$, bien que ce dernier corresponde à un nombre moyen de citations par entrée (mais également à un nombre d'entrées).

La corrélation élevée des classements en nombre de citations totales et par entrée, supérieure à 
celle de ces derniers avec les indices $\mathrm{H}$ et $\mathrm{G}$, montre que les institutions recevant beaucoup de citations sont celles où la qualité de chacune des publications est elle même élevée. Recevoir beaucoup de citations ne consiste pas seulement à effectuer beaucoup de travaux mais à également avoir une qualité élevée pour chacun d'entre eux.

Une originalité de notre travail est de permettre de comparer les indices de citations (GS ici) avec des indices très différents dans leur nature, fondés sur la qualité moyenne des supports de publication et non la qualité de chaque publication prise isolément. Il s'avère que la hiérarchie des centres évaluée selon les approches GS, quelles qu'elles soient, est relativement proche de la hiérarchie obtenue via $\mathrm{Clm}$, avec des corrélations de rang supérieures à 0.7 que ce soit pour les citations totales reçues ou les indices H ou G. Des corrélations légèrement plus faibles sont observées avec les scores par entrée, de façon relativement naturelle puisque les scores Clm correspondent à des volumes de publications. Ainsi, utiliser la qualité des journaux comme prédicteur de la qualité des publications, en tous les cas du nombre de citations qu'elles reçoivent, semble constituer une stratégie tout à fait pertinente. Les corrélations sont légèrement plus faibles avec $\mathrm{Clh}$, quoique restant largement positives. Ainsi, les indicateurs de citations GS semblent moins discriminants que ne le sont les indicateurs $\mathrm{Clh}$, et se rapprochent plus des indicateurs $\mathrm{Clm}$. Notons que des explications de ce résultat peuvent se trouver dans le fait que les citations GS ne prennent pas en compte la qualité du support citant, alors que les indicateurs de citations des journaux à partir desquels $\mathrm{Clm}$ et $\mathrm{Clh}$ sont construits le font. Ces corrélations sont légèrement moins élevées en ce qui concerne la hiérarchie des centres selon leurs scores par chercheur, ce qui s'explique sans doute par la plus grande variabilité globale de ceux-ci.

TABLE 5 - Corrélations entre rangs des centres, scores totaux, T=Dégressif

\begin{tabular}{|c|c|c|c|c|c|c|c|c|c|c|c|c|}
\hline & CtI & $\mathrm{Ct}$ & CeI & $\mathrm{Ce}$ & Cp1 & $\mathrm{Cp}$ & $\mathrm{H}_{1}$ & $\mathrm{H}$ & $\mathrm{G}_{1}$ & $\mathrm{G}$ & $\mathrm{Clm}$ & $\mathrm{Clh}$ \\
\hline $\mathrm{Ct}_{1}$ & 1 & 0.99 & 0.85 & 0.86 & 0.86 & 0.86 & 0.95 & 0.94 & 0.95 & 0.97 & 0.84 & 0.72 \\
\hline $\mathrm{Ct}$ & & 1 & 0.84 & 0.86 & 0.85 & 0.85 & 0.95 & 0.96 & 0.94 & 0.96 & 0.85 & 0.72 \\
\hline Ce1 & & & 1 & 0.99 & 0.98 & 0.98 & 0.77 & 0.78 & 0.80 & 0.81 & 0.76 & 0.64 \\
\hline $\mathrm{Ce}$ & & & & 1 & 0.97 & 0.98 & 0.79 & 0.80 & 0.81 & 0.82 & 0.77 & 0.65 \\
\hline Cp1 & & & & & 1 & $I$ & 0.77 & 0.77 & 0.79 & 0.80 & 0.75 & 0.61 \\
\hline $\mathrm{Cp}$ & & & & & & $I$ & 0.78 & 0.78 & 0.79 & 0.80 & 0.76 & 0.62 \\
\hline $\mathrm{H}_{1}$ & & & & & & & 1 & 0.96 & 0.93 & 0.95 & 0.85 & 0.76 \\
\hline $\mathrm{H}$ & & & & & & & & 1 & 0.92 & 0.93 & 0.86 & 0.74 \\
\hline $\mathrm{G}_{1}$ & & & & & & & & & 1 & 0.99 & 0.82 & 0.75 \\
\hline G & & & & & & & & & & 1 & 0.83 & 0.75 \\
\hline $\mathrm{Clm}$ & & & & & & & & & & & 1 & 0.90 \\
\hline Clh & & & & & & & & & & & & 1 \\
\hline
\end{tabular}

Les résultats observés au niveau des centres sont confirmés au niveau des universités, les classements desquelles sont en général encore plus corrélés que ceux des centres. Notamment, les corrélations des scores en volume de citations ou volume $\mathrm{Clm}$ sont supérieures à 0.79 , légèrement infé- 
TABLE 6 - Corrélations entre rangs des centres, scores par chercheur, T=Dégressif

\begin{tabular}{|c|c|c|c|c|c|c|c|c|c|c|c|c|}
\hline & Ct1 & $\mathrm{Ct}$ & Ce1 & $\mathrm{Ce}$ & Cp1 & $\mathrm{Cp}$ & $\mathrm{H}_{1}$ & $\mathrm{H}$ & GI & $\mathrm{G}$ & $\mathrm{Clm}$ & Clh \\
\hline $\mathrm{Ct} 1$ & 1 & 0.98 & 0.82 & 0.81 & 0.83 & 0.83 & 0.93 & 0.91 & 0.95 & 0.91 & 0.71 & 0.65 \\
\hline $\mathrm{Ct}$ & & 1 & 0.80 & 0.80 & 0.80 & 0.80 & 0.93 & 0.94 & 0.95 & 0.93 & 0.71 & 0.63 \\
\hline Ce1 & & & 1 & 0.99 & 0.94 & 0.95 & 0.79 & 0.76 & 0.76 & 0.71 & 0.63 & 0.61 \\
\hline $\mathrm{Ce}$ & & & & 1 & 0.93 & 0.94 & 0.79 & 0.76 & 0.76 & 0.70 & 0.63 & 0.61 \\
\hline Cp1 & & & & & 1 & 0.99 & 0.79 & 0.76 & 0.78 & 0.73 & 0.59 & 0.55 \\
\hline $\mathrm{Cp}$ & & & & & & 1 & 0.79 & 0.76 & 0.78 & 0.73 & 0.59 & 0.55 \\
\hline $\mathrm{H}_{1}$ & & & & & & & 1 & 0.98 & 0.97 & 0.97 & 0.73 & 0.63 \\
\hline $\mathrm{H}$ & & & & & & & & 1 & 0.95 & 0.96 & 0.72 & 0.61 \\
\hline $\mathrm{G}_{1}$ & & & & & & & & & 1 & 0.97 & 0.72 & 0.64 \\
\hline G & & & & & & & & & & 1 & 0.71 & 0.59 \\
\hline $\mathrm{Clm}$ & & & & & & & & & & & 1 & 0.91 \\
\hline Clh & & & & & & & & & & & & $I$ \\
\hline
\end{tabular}

Notes : $\mathrm{Ct}=$ citations totales $; \mathrm{Ce}=$ citations par entrée $; \mathrm{Cp}=$ citations par entrée ayant au moins une citation; $\mathrm{H}=$ Indice $\mathrm{H} ; \mathrm{G}=$ Indice $\mathrm{G}$; 1 indique que le nombre de co-auteurs n'est pas pris en compte. Clm et Clh correspondent aux indices Econlit plus (Clh) ou moins (Clm) sélectifs.

rieures pour les volumes par chercheur. En désagrégeant l'information au contraire, c'est-à-dire en se plaçant cette fois-ci au niveau des chercheurs, les corrélations entre classements GS restent très élevées, seulement très légèrement plus faibles qu'entre centres et universités. Les corrélations entre scores GS et scores $\mathrm{Clm}$, et encore plus $\mathrm{Clh}$, deviennent plus faibles, ce qui reflète un résultat relativement standard relatif au fait que l'agrégation par centre ou université tend à rendre la hiérarchie obtenue relativement moins sensible à la méthode utilisée. Les corrélations entre scores en volume restent néanmoins toutes supérieures à o.6. Le détail de ces corrélations aux niveaux universités et chercheurs peut être trouvé dans Bosquet et Combes (2011a).

Finalement, les Tableaux 7 et 8 présentent également des corrélations entre centres, mais cette fois-ci lorsque la période d'étude est modifiée. Là encore, l'on est surtout frappé par les valeurs très élevées prises par ces corrélations. L'ordre des centres est donc remarquablement stable selon les différentes périodes d'étude, et il en va de même pour les universités et les chercheurs comme le montrent les corrélations présentées dans Bosquet et Combes (2011a).

TABLE 7 - Corrélations des rangs des centres entre périodes, scores totaux

\begin{tabular}{|c|c|c|c|c|c|c|c|c|c|}
\hline \multirow[b]{3}{*}{$\mathrm{Ct}, \mathrm{T}=\mathrm{All}$} & \multicolumn{5}{|c|}{$\mathrm{Ct}$} & \multicolumn{4}{|c|}{$\bar{G}$} \\
\hline & $\mathrm{T}=\mathrm{All}$ & $\mathrm{T}=5$ ans & $\mathrm{T}=\mathrm{An}$. & T=Dég. & $\mathrm{T}=$ Car. & $\mathrm{T}=\mathrm{All}$ & $\mathrm{T}=5$ ans & $\mathrm{T}=\mathrm{An}$ & $\mathrm{T}=$ Dég. \\
\hline & 1 & 0.91 & 0.98 & 0.96 & 0.96 & 0.95 & 0.87 & 0.88 & 0.92 \\
\hline $\mathrm{Ct}, \mathrm{T}=5$ ans & & 1 & 0.96 & 0.98 & 0.94 & 0.91 & 0.96 & 0.92 & 0.95 \\
\hline $\mathrm{Ct}, \mathrm{T}=$ Annuel & & & 1 & 1 & 0.97 & 0.95 & 0.92 & 0.93 & 0.95 \\
\hline $\mathrm{Ct}, \mathrm{T}=$ Dégressif & & & & 1 & 0.97 & 0.94 & 0.93 & 0.93 & 0.96 \\
\hline $\mathrm{Ct}, \mathrm{T}=$ Carrière & & & & & 1 & 0.93 & 0.91 & 0.91 & 0.94 \\
\hline $\mathrm{G}, \mathrm{T}=\mathrm{All}$ & & & & & & 1 & 0.92 & 0.96 & 0.97 \\
\hline $\mathrm{G}, \mathrm{T}=5$ ans & & & & & & & 1 & 0.96 & 0.98 \\
\hline $\mathrm{G}, \mathrm{T}=$ Annuel & & & & & & & & 1 & 0.98 \\
\hline G, T=Dégressif & & & & & & & & & 1 \\
\hline
\end{tabular}

Notes : $\mathrm{Ct}=$ citations totales $; \mathrm{G}=$ Indice $\mathrm{G}$. 
TABLE 8 - Corrélations des rangs des centres entre périodes, scores par chercheur

\begin{tabular}{|c|c|c|c|c|c|c|c|c|c|}
\hline & \multicolumn{5}{|c|}{$\mathrm{Ct}$} & \multicolumn{4}{|c|}{$\mathrm{G}$} \\
\hline & $\mathrm{T}=$ All & $\mathrm{T}=5$ ans & $\mathrm{T}=\mathrm{An}$. & $\mathrm{T}=$ Dég. & $\mathrm{T}=$ Car. & $\mathrm{T}=\mathrm{All}$ & $\mathrm{T}=5$ ans & $\mathrm{T}=\mathrm{An}$. & $\mathrm{T}=$ Dég. \\
\hline $\mathrm{Ct}, \mathrm{T}=\mathrm{All}$ & 1 & 0.83 & 0.96 & 0.94 & 0.93 & 0.93 & 0.83 & 0.91 & 0.87 \\
\hline $\mathrm{Ct}, \mathrm{T}=5 \mathrm{ans}$ & & 1 & 0.93 & 0.95 & 0.88 & 0.86 & 0.92 & 0.90 & 0.89 \\
\hline $\mathrm{Ct}, \mathrm{T}=$ Annuel & & & 1 & 0.99 & 0.95 & 0.93 & 0.90 & 0.95 & 0.92 \\
\hline $\mathrm{Ct}, \mathrm{T}=$ Dégressif & & & & 1 & 0.94 & 0.93 & 0.92 & 0.95 & 0.93 \\
\hline $\mathrm{Ct}, \mathrm{T}=$ Carrière & & & & & 1 & 0.89 & 0.84 & 0.89 & 0.86 \\
\hline $\mathrm{G}, \mathrm{T}=\mathrm{All}$ & & & & & & 1 & 0.91 & 0.97 & 0.94 \\
\hline $\mathrm{G}, \mathrm{T}=5$ ans & & & & & & & 1 & 0.93 & 0.98 \\
\hline $\mathrm{G}, \mathrm{T}=$ Annuel & & & & & & & & 1 & 0.95 \\
\hline$G, T=$ Dégressif & & & & & & & & & 1 \\
\hline
\end{tabular}

Notes : $\mathrm{Ct}=$ citations totales; $\mathrm{G}=$ Indice $\mathrm{G}$.

\section{Disparités de citations et tendances nationales}

\subsection{Citations Google Scholar de la recherche en économie}

Comme l'indique le Tableau 9, les chercheurs localisés en France reçoivent ensemble quasiment 270000 citations GS en janvier 2010, 47000 pour leurs entrées de la période 2004-2008. Chaque entrée a en moyenne presque 7 citations, le double pour celles en ayant au moins une. La France a un indice $\mathrm{H}$ d'environ 180 , soit 180 entrées ayant au moins 180 citations, sachant que, comme nous le verrons plus loin, le chercheur au dernier centile a un indice $\mathrm{H}$ d'environ 17, et la France a environ 500 entrées ayant en moyenne 500 citations (indice G). Sur 5 ans, ces deux indices sont divisés par un peu plus que deux. La France obtient environ 34000 citations GS par an, et une nouvelle entrée obtient en moyenne plus d'une citation par année, 2 une fois qu'elle en a obtenue au moins une. Elle a environ 50 entrées recevant au moins 50 citations par an en moyenne.

TABLE 9 - Scores France entière

\begin{tabular}{lrrrrr}
\hline \hline & $\mathrm{Ct}$ & $\mathrm{Ce}$ & $\mathrm{Cp}$ & $\mathrm{H}$ & $\mathrm{G}$ \\
\hline $\mathrm{T}=$ All & 265578.3 & 6.7 & 13.9 & 177.9 & 485.3 \\
$\mathrm{~T}=5$ ans & 46619.0 & 4.8 & 9.1 & 72.9 & 217.4 \\
$\mathrm{~T}=$ Annuel & 34315.5 & 1.2 & 2.0 & 48.2 & 134.5 \\
$\mathrm{~T}=$ Dégressif & 50896.0 & 5.6 & 10.4 & 50.3 & 218.5 \\
\hline
\end{tabular}

Le Tableau 10 indique que sur l'ensemble de leur carrière, seuls $6.2 \%$ des chercheurs n'ont pas d'entrée GS et $15 \%$ n'en ont pas ayant au moins une citation, alors que $26.6 \%$ n'ont pas de publication Econlit. Sur les cinq dernières années, alors que 45.3\% n'ont pas de publication Econlit, seulement 22.3\% n'ont pas d'entrée GS et 34.3\% n'en ont pas avec au moins une citation. Ainsi, la production accrue des français en termes d'entrées GS n'est pas simplement due au fait que les chercheurs publiant selon Econlit ont plus d'entrées GS que de publications Econlit mais aussi au fait que la part des non publiant est plus faible selon GS. Cela est relativement "normal", puisque le support est élargi, mais nous disposons donc ici d'une mesure précise de l'élargissement produit. On trouvera 
dans Bosquet et Combes (2011a) ces parts de publiant pour chacune des universités de notre étude.

TABLE 10 - Parts de chercheurs publiant, France entière

\begin{tabular}{lrrr}
\hline \hline & Pub. GS & Cit. GS & Pub. EL \\
\hline $\mathrm{T}=$ All & 93.8 & 85.0 & 73.4 \\
$\mathrm{~T}=5$ ans & 77.7 & 65.7 & 54.7 \\
\hline
\end{tabular}

Etant donné ces résultats, ainsi que les corrélations obtenues entre classements, nous nous concentrons principalement dans la suite de cet article sur deux indices GS relativement différents, à savoir le nombre de citations totales et l'indice G, qui sont systématiquement comparés à Clm.

\subsection{Disparités entre chercheurs, centres et universités}

Les disparités de publication des chercheurs sont grandes, ce qui constitue un fait général relativement ancien et solidement établi depuis Lotka (1926). Plus intéressant est donc la possibilité de comparer le degré de disparités selon l'outil de mesure utilisé, ce qui correspond à l'un des objets de cet article. Le Tableau 11 compare pour la période $\mathrm{T}=$ Dégressif un certain nombre d'indicateurs de disparités. Il s'avère que l'indice G est relativement peu discriminant, en tous les cas bien moins que le nombre total de citations reçues, ce qui apparaît pour quasiment toutes les mesures de disparités calculées ici. Par exemple, alors que le rapport inter-quartiles entre universités est de 2 pour l'indice $G$, il est de quasiment 13 en termes de citations totales, et l'on obtient des ratios de 5 et 32 pour les rapports inter-déciles. Les écarts sont similaires au niveau des centres.

Il est également intéressant de noter que l'indice Clm est en fait intermédiaire en termes de disparité entre les indicateurs de nombre total de citations reçues et l'indice G. Cela est vrai non seulement sur le haut de la distribution (rapport $\mathrm{P}_{90} / \mathrm{P}_{50}$ ) mais également sur le bas (rapport $\mathrm{P}_{50} / \mathrm{P}_{10}$ ). Cela est plus surprenant, puisque, au niveau des chercheurs, Clm semble peu discriminant sur le bas de la distribution, un grand nombre d'entre eux ayant en fait un score nul, alors que les scores GS continuent à décroître de façon relativement continue. On trouvera dans Bosquet et Combes (2011a) des tableaux identiques pour $\mathrm{T}=\mathrm{All}$ et $\mathrm{T}=5$ ans à partir desquels des conclusions similaires sont obtenues. Dans la section 3.3 décrivant la distribution d'un grand nombre de scores, des comparaisons supplémentaires de disparités des différents indices sont données.

Un dernier indicateur de concentration de la production intra-institution est donné par la part des citations du chercheur le plus cité de l'institution (Tableau 12). L'approche GS, avec des parts moyennes de ce chercheur aux alentours de $40 \%$, conduit à un poids encore plus élevé de celui-ci dans la production de son centre que l'approche Econlit, ce que l'on retrouve en tout point de la distribution de cette grandeur. 
TABLE 11 - Indicateurs de dispersion des scores, T=Dégressif

\begin{tabular}{|c|c|c|c|c|c|c|c|c|c|c|c|c|c|c|c|c|c|c|c|c|c|}
\hline & & nter & & uni & $\begin{array}{l}\text { vers } \\
\text { ater }\end{array}$ & & & intr & & & nter & & & $\begin{array}{l}\text { entre } \\
\text { ater }\end{array}$ & & & intr & & che & erche & eur \\
\hline & $\mathrm{Ct}$ & G & $\mathrm{Clm}$ & $\mathrm{Ct}$ & G & $\mathrm{Clm}$ & $\mathrm{Ct}$ & $\mathrm{G}$ & $\mathrm{Clm}$ & $\mathrm{Ct}$ & G & $\mathrm{Clm}$ & $\mathrm{Ct}$ & $\mathrm{G}$ & $\mathrm{Clm}$ & $\mathrm{Ct}$ & G & $\mathrm{Clm}$ & $\mathrm{Ct}$ & G & $\mathrm{Clm}$ \\
\hline coef. var. & 2.4 & 0.6 & 1.2 & 2.2 & 0.8 & 0.9 & 1.8 & 1.2 & 1.6 & 2.1 & 0.7 & 1.4 & 2.0 & 0.8 & 1.2 & 1.8 & 1.2 & 1.6 & 4.2 & 1.3 & 2.6 \\
\hline $\mathrm{p} 75 / \mathrm{p} 25$ & 12.8 & 2.0 & 3.0 & $5 \cdot 4$ & 2.7 & 2.7 & . & . & . & 6.3 & 2.1 & $3 \cdot 3$ & 4.9 & 2.3 & 3.6 & . & . & . & 20.9 & $7 \cdot 7$ & \\
\hline p9o/p1o & 32.3 & 5.2 & 12.4 & 28.6 & 5.6 & 8.0 & . & . & . & 29.8 & 5.2 & 12.4 & 27.0 & 4.7 & $9 \cdot 7$ & . & . & . & . & . & \\
\hline $\mathrm{p}_{50} / \mathrm{p}_{10}$ & 5.1 & 2.4 & 3.1 & 4.1 & 2.3 & 2.6 & . & . & . & $4 \cdot 7$ & 2.4 & 2.9 & 4.1 & 2.0 & 2.9 & . & . & . & . & . & \\
\hline $\mathrm{p} 90 / \mathrm{p} 50$ & 6.3 & 2.2 & 4.1 & 7.0 & 2.4 & 3.1 & . & . & . & 6.3 & 2.2 & 4.2 & 6.6 & 2.4 & $3 \cdot 3$ & . & . & . & 10.4 & 4.1 & $14 \cdot 3$ \\
\hline
\end{tabular}

Note : "inter t." : dispersion entre universités ou centres des scores totaux, "inter c." : dispersion entre universités ou centres des scores par chercheur, "intra" : dispersion intra-universités ou centres, coeff. var. : coefficient de variation, p75/p25 : rapport inter-quartile, p9o/p10 : rapport dernier/premier décile, p5o/p10 : rapport médiane/premier décile, p9o/p5o : rapport dernier décile/médiane.

TABLE 12 - Distribution de la part du chercheur le plus cité dans le centre (\%)

\begin{tabular}{rrrrrrrrrrrr}
\hline \hline & moy. & c.v. & P1 & P5 $_{5}$ & P10 & P25 $_{20}$ & P5 $_{50}$ & P$_{75}$ & P90 & P95 & P99 \\
Ct, T=All & 37.2 & 0.5 & 9.6 & 14.3 & 17.2 & 21.8 & 33.5 & 46.3 & 64.4 & 69.9 & 86.2 \\
Ct, T=5 ans & 41.0 & 0.5 & 9.7 & 14.1 & 18.0 & 25.4 & 33.2 & 53.0 & 75.0 & 79.2 & 91.7 \\
& & & & & & & & & & & \\
Clm, T=All & 29.6 & 0.6 & 8.3 & 11.1 & 13.5 & 18.3 & 24.6 & 37.4 & 49.6 & 58.3 & 83.5 \\
Clm, T=5 ans & 34.4 & 0.6 & 5.9 & 9.9 & 15.9 & 20.7 & 28.5 & 42.5 & 57.7 & 83.5 & 100.0 \\
\hline
\end{tabular}

Notes : $\mathrm{Ct}=$ citations totales ; Clm est un indice de publication Econlit. Lecture : le chercheur qui est le plus cité dans chaque laboratoire pèse en moyenne $37,2 \%$ des citations totales du laboratoire. Il y a 10\% des laboratoires français de recherche en économie où le meilleur chercheur pèse moins de $17,2 \%$ des citations.

\subsection{Distributions des scores des chercheurs}

Cette section présente la distribution des scores des chercheurs, l'intérêt étant aussi de permettre à un chercheur de se situer dans la distribution nationale. ${ }^{3}$ Le caractère plus ou moins sélectif des différentes approches apparaît clairement. Sont présentés dans les Tableau 13 et 14 les distributions pour l'ensemble des citations reçues pour $\mathrm{T}=\mathrm{All}$ et $\mathrm{T}=5$ ans respectivement, celles pour $\mathrm{T}=$ Dégressif et $\mathrm{T}=$ Carrière étant données dans Bosquet et Combes (2011a).

En termes de disparités, il est bien confirmé que si l'indice Clm est plus sélectif que les indices $\mathrm{H}$, G ou par entrée, il l'est moins que ceux en nombre total de citations et pourrait constituer à ce titre une bonne synthèse entre ceux-ci. Cela n'est plus le cas pour Clh qui est le plus sélectif de tous. On observe que les indices GS permettent de mieux discriminer le bas de la distribution pour lequel des scores nuls, et donc non discriminants, sont obtenus selon $\mathrm{Clm}$ et $\mathrm{Clh}$. Le nombre total de citations reçues est très sélectif. Il est divisé par un facteur 4 lorsque l'on passe du dernier centile au 95ème, puis encore d'un facteur deux en passant de celui-ci au dernier décile. Il y a un facteur 100 entre le chercheur médian et celui situé au dernier centile, pour un facteur 70 en $\mathrm{Clm}$, et de l'ordre de 8 et 12 respectivement pour les indices $H$ et G. L'ensemble de tous les travaux du chercheur médian

3. Sans oublier que le nombre de citations GS est en expansion constante et que la présente étude se base sur des données de janvier 2010 . 
ne représente un stock de citations équivalent reçues seul que de 14.67. Il a 2 articles ayant reçu au moins 2 citations (indice $\mathrm{H}$ ) et 4 qui en ont reçu en moyenne 4 . Le chercheur au dernier centile a un stock d'environ 17 articles ayant reçu au moins 17 citations, mais tout de même 48 articles en ayant reçu en moyenne 48.

Sur les autres périodes de temps, les scores obtenus deviennent rapidement très faibles, comme le montre le Tableau 14 pour $\mathrm{T}=5$ ans et les tableaux donnés dans Bosquet et Combes (2011a) pour $\mathrm{T}=$ Dégressif et $\mathrm{T}=$ Carrière, reflétant le fait qu'être cité nécessite en tout état de cause du temps, ne serait-ce que par les délais de publications des articles citant même si ce temps devrait être réduit pour des citations sur Internet. Le chercheur au dernier centile bénéficie tout de même de 232 citations équivalent reçues seuls en janvier 2010 pour ses travaux des 5 années 2004-2008, ses 6 articles de la période les plus cités sont cités au moins 6 fois et il a environ 16 articles cités en moyenne 16 fois.

TABLE 13 - Distributions des scores des chercheurs, T=All

\begin{tabular}{|c|c|c|c|c|c|c|c|c|c|c|c|}
\hline index & mean & c.v. & P10 & $P_{15}$ & $\mathrm{P}_{20}$ & $\mathrm{P}_{25}$ & $\mathrm{P}_{50}$ & $\mathrm{P}_{75}$ & P9o & P95 & P99 \\
\hline $\mathrm{Ct} 1$ & 171.06 & 5.86 & o & 1.00 & 2.00 & 4.00 & 24.00 & 99.00 & 342.00 & 639.00 & 2378.00 \\
\hline $\mathrm{Ct}$ & $97 \cdot 33$ & 6.26 & o & 0.20 & 1.00 & 2.50 & 14.67 & 53.79 & 181.50 & 350.30 & 1387.92 \\
\hline Ce1 & $3 \cdot 79$ & 1.80 & $\mathrm{O}$ & 0.07 & 0.40 & 0.64 & 2.00 & $4 \cdot 39$ & 9.07 & 13.24 & 25.93 \\
\hline $\mathrm{Ce}$ & 3.63 & 1.86 & $\mathrm{o}$ & 0.03 & 0.39 & 0.60 & 1.87 & 4.27 & 8.37 & 12.75 & 27.00 \\
\hline Cp1 & 7.00 & 1.30 & $\mathrm{O}$ & 1.00 & 1.50 & 2.00 & 4.67 & 8.82 & $15 \cdot 50$ & 21.84 & 38.34 \\
\hline Cp & 6.73 & 1.33 & o & 1.00 & 1.50 & 2.00 & $4 \cdot 50$ & 8.50 & 14.82 & 20.97 & 39.40 \\
\hline $\mathrm{H}_{1}$ & 3.94 & 1.21 & o & 1.00 & 1.00 & 1.00 & 3.00 & 5.00 & 9.00 & 13.00 & 23.00 \\
\hline $\mathrm{H}$ & 2.77 & 1.21 & o & 0.17 & 0.50 & 0.83 & 2.00 & $3 \cdot 5^{8}$ & 6.33 & 8.67 & 16.85 \\
\hline $\mathrm{G}_{1}$ & 7.14 & 1.40 & o & 1.00 & 1.00 & 2.00 & 4.00 & 9.00 & 17.00 & 23.00 & 48.00 \\
\hline G & 6.97 & 1.39 & $\mathrm{o}$ & 0.20 & 1.00 & 1.33 & 4.00 & 8.83 & 16.50 & 22.83 & 48.20 \\
\hline Clm & 53.92 & $3 \cdot 53$ & o & o & o & o & 11.35 & 40.50 & 110.22 & 205.84 & 771.55 \\
\hline $\mathrm{Clh}$ & 13.75 & 7.45 & $\mathrm{O}$ & $\mathrm{O}$ & $\mathrm{O}$ & $\mathrm{O}$ & 0.05 & 0.76 & 11.90 & $44 \cdot 51$ & 370.33 \\
\hline
\end{tabular}

\subsection{Citations en fonction de l'âge}

La distribution des âges des chercheurs (particulière par sa bi-modalité illustrée dans Bosquet, Combes, et Linnemer (2010) avec une sur-représentation des chercheurs d'environ 38 et 60 ans) est susceptible d'évoluer sensiblement dans le temps. De plus, les différentes générations de chercheurs de notre base n'ont pas fait face aux mêmes incitations à publier et aux mêmes supports pour le faire. Nous présentons, néanmoins, ici les différences de publication des chercheurs français en fonction de leur âge. Afin de rendre la comparaison pertinente et contourner les effets de stocks, nous sommes obligés de nous placer sur une période identique pour tous et relativement courte, soit $\mathrm{T}=5$ ans, même si les résultats pour les autres périodes sont donnés dans Bosquet et Combes (2011a). 
TABLE 14 - Distributions des scores des chercheurs, $\mathrm{T}=5$ ans

\begin{tabular}{|c|c|c|c|c|c|c|c|c|c|c|c|}
\hline index & mean & c.v. & P10 & $P_{15}$ & $\mathrm{P}_{20}$ & $\mathrm{P}_{25}$ & $\mathrm{P}_{50}$ & $P_{75}$ & P9o & P95 & P99 \\
\hline CtI & 33.95 & 3.55 & $\mathrm{O}$ & o & $\mathrm{O}$ & o & 4.00 & 23.00 & 83.00 & 142.00 & 424.00 \\
\hline $\mathrm{Ct}$ & 17.23 & 3.78 & o & o & o & o & 2.50 & 12.00 & 38.30 & 68.83 & 232.07 \\
\hline CeI & 2.78 & 1.82 & $\mathrm{O}$ & o & o & $\mathrm{O}$ & 1.00 & $3 \cdot 38$ & $7 \cdot 38$ & 11.20 & 24.25 \\
\hline $\mathrm{Ce}$ & 2.75 & 1.90 & $\mathrm{O}$ & $\mathrm{O}$ & $\mathrm{O}$ & $\mathrm{O}$ & 1.00 & 3.22 & 7.11 & 10.87 & 25.29 \\
\hline Сp 1 & 4.40 & 1.72 & $\mathrm{O}$ & $\mathrm{O}$ & O & $\mathrm{O}$ & 2.00 & $5 \cdot 75$ & 11.00 & 16.00 & 35.00 \\
\hline $\mathrm{Cp}$ & $4 \cdot 37$ & 1.79 & o & o & $\mathrm{O}$ & o & 2.00 & $5 \cdot 50$ & 10.79 & 15.71 & 34.52 \\
\hline $\mathrm{HI}_{1}$ & 1.75 & 1.23 & o & o & o & o & 1.00 & 2.00 & 4.00 & 6.00 & 10.00 \\
\hline $\mathrm{H}$ & 1.11 & 1.25 & $\mathrm{O}$ & o & O & $\mathrm{O}$ & 0.75 & 1.75 & 2.83 & 3.67 & 6.12 \\
\hline GI & 2.95 & 1.46 & $\mathrm{O}$ & O & O & $\mathrm{O}$ & 2.00 & 4.00 & 8.00 & 11.00 & 20.00 \\
\hline G & 2.41 & 1.48 & o & o & o & o & 1.17 & $3 \cdot 33$ & 6.57 & 9.00 & 15.98 \\
\hline $\mathrm{Clm}$ & 17.88 & 2.48 & o & o & o & o & 2.56 & 16.92 & 48.97 & 86.98 & 200.41 \\
\hline $\mathrm{Clh}$ & $3 \cdot 39$ & 5.66 & $\mathrm{O}$ & $\mathrm{O}$ & $\mathrm{O}$ & $\mathrm{O}$ & 0.01 & 0.19 & 3.42 & 16.23 & $74 \cdot 32$ \\
\hline
\end{tabular}

Notes : c.v. = coefficient of variation $; \mathrm{Ct}=$ citations totales $; \mathrm{Ce}=$ citations par entrée ; $\mathrm{Cp}=$ citations par entrée ayant au moins une citation; $\mathrm{H}=$ Indice $H$; $\mathrm{G}=$ Indice $\mathrm{G}$; 1 indique que le nombre de co-auteurs n'est pas pris en compte. $\mathrm{Clm}$ et $\mathrm{Clh}$ sont les indices Econlit plus (Clh) ou moins (Clm) sélectifs.

Le résultat marquant est que l'on retrouve une courbe en cloche de la productivité des chercheurs pour les indices GS, quels qu'ils soient, comme on l'obtient pour les indices Clm et Clh. Le pic de productivité n'est pas atteint tout à fait au même âge selon les indices utilisés, le plus tardif étant observé pour les citations totales alors que pour les citations par article il est un des plus précoces : les chercheurs jeunes semblent privilégier la qualité pour chaque article, les plus âgés ayant plus tendance à produire aussi des articles à diffusion plus restreinte. Pour les indices $\mathrm{H}$ et $\mathrm{G}$, cette variation au cours du cycle de vie des chercheurs est moins marquée, alors qu'elle est quasiment maximale pour les indices $\mathrm{Clm}$ et $\mathrm{Clh}$.

TABLE 15 - Scores par chercheur par classe d'âge, $T=5$ ans

\begin{tabular}{rrrrrrrrr}
\hline \hline & $26-30$ & $31-35$ & $36-40$ & $41-45$ & $46-50$ & $51-55$ & $56-60$ & $>60$ \\
Ct1 & 19.71 & 32.76 & 35.98 & 40.05 & 44.34 & 50.09 & 19.77 & 24.55 \\
Ct & 10.43 & 16.81 & 17.77 & 18.81 & 21.93 & 25.76 & 10.70 & 13.45 \\
Ce1 & 2.52 & 3.54 & 3.35 & 3.28 & 2.61 & 2.44 & 1.71 & 2.22 \\
Ce & 2.33 & 3.45 & 3.35 & 3.25 & 2.51 & 2.43 & 1.69 & 2.24 \\
Cp1 & 4.11 & 5.39 & 5.07 & 5.37 & 4.13 & 3.71 & 2.76 & 3.87 \\
Cp & 4.02 & 5.30 & 5.02 & 5.30 & 4.05 & 3.68 & 2.75 & 3.94 \\
H1 & 1.73 & 2.12 & 2.06 & 1.89 & 1.88 & 1.77 & 1.28 & 1.21 \\
H & 1.18 & 1.40 & 1.27 & 1.18 & 1.15 & 1.10 & 0.85 & 0.78 \\
G1 & 2.75 & 3.45 & 3.37 & 3.28 & 3.28 & 3.11 & 2.07 & 2.16 \\
G & 2.38 & 2.89 & 2.67 & 2.60 & 2.67 & 2.58 & 1.79 & 1.80 \\
Clm & 16.62 & 24.05 & 22.17 & 23.17 & 20.53 & 16.32 & 9.74 & 9.29 \\
Clh & 1.70 & 2.78 & 4.17 & 5.56 & 5.02 & 4.61 & 1.18 & 1.28
\end{tabular}

Notes : $\mathrm{Ct}=$ citations totales $; \mathrm{Ce}=$ citations par entrée $; \mathrm{Cp}=$ citations par entrée ayant au moins une citation; $H=$ Indice $H$; $G$ = Indice G; 1 indique que le nombre de co-auteurs n'est pas pris en compte. Clm et $\mathrm{Clh}$ sont les indices Econlit plus (Clh) ou moins (Clm) sélectifs. 


\subsection{Disparités entre statuts}

Les chercheurs français n'ont pas tous le même statut avec des temps consacrés à l'activité de publication d'articles de recherche différents. Les Tableaux 16 et 17 mesurent respectivement les citations totales et indices $G$ en volume et par chercheur pour chacun des statuts de notre base en indiquant les variations par rapport à la hiérarchie selon Clm. Les statuts apparaissent en gras italique s'ils progressent d'au moins 3 rangs, et en italique simple s'ils régressent d'au moins 3 rangs entre les deux types d'approches, la variation exacte étant donnée entre parenthèses.

Il apparaît que les quatre ou cinq statuts les plus productifs selon les indices GS restent identiques à ceux identifiés au moyen de $\mathrm{Clm}$ et $\mathrm{Clh}$. En revanche, il est intéressant de noter que progressent selon les mesures GS les statuts qui ne sont pas au coeur de notre analyse, comme peuvent l'être les maîtres de conférence de la section 5 ou les chargés de recherche de la section 37. Cela s'explique relativement aisément par le fait que GS élargit le spectre de publications considérées, notamment en direction d'autres disciplines, connexes à l'économie tout de même comme la gestion, la sociologie, l'histoire ou la géographie etc. Ainsi, les "assimilés" professeurs ou chargés de recherche, en poste dans des grandes écoles, parfois de commerce ou dans des administrations, les chercheurs Cnrs non section 37 connaissent-ils les progressions les plus importantes.

La hiérarchie des statuts est très proche lorsqu'on la mesure à l'aide de l'indice G moyen ou du nombre de citations par chercheur (colonne de droite des Tableaux 16 et 17). Elle varie plus lorsqu'on compare le nombre de citations totales reçues et l'indice G de l'ensemble du statut (colonne de gauche des Tableaux 16 et 17), les écarts se resserrant d'une part et les statuts étant très productifs en moyenne remontant en termes de G grâce, probablement, à leurs meilleurs éléments. 
TABLE 16 - Statuts, Citations totales, T=Dégressif

\begin{tabular}{lcrr}
\hline \hline statut & rg. & tot. & nor. \\
\hline PR 05 (568)(0) & 1 & 16747.8 & 100.0 \\
MCF 05 (1282)(o) & 2 & 7253.4 & $43 \cdot 3$ \\
DR cnrs (84)(0) & 3 & 5597.6 & 33.4 \\
Ponts et C. (7)(+1) & 4 & 4254.8 & 25.4 \\
A-PR (97)(+1) & 5 & 3859.1 & 23.0 \\
PR non 05 (158)(+2) & 6 & 3406.6 & 20.3 \\
DE ehess (13)(+2) & 7 & 2654.0 & 15.9 \\
CR cnrs (102)(-4) & 8 & 2299.2 & 13.7 \\
DR inra (56)(+1) & 9 & 1493.2 & 8.9 \\
Insee (22)(+2) & 10 & 1368.9 & 8.2 \\
CR inra (89)(-4) & 11 & 1268.7 & 7.6 \\
MCF non 05(260)(-1) & 12 & 1080.6 & 6.5 \\
A-CR (21)(0) & 13 & 743.2 & 4.4 \\
DR cnrs non 37 (10)(+5) & 14 & 253.3 & 1.5 \\
Autre (13)(+2) & 15 & 249.8 & 1.5 \\
Insee non EC (27)(-2) & 16 & 242.1 & 1.5 \\
CR cnrs non 37(9)(+1) & 17 & 157.9 & 0.9 \\
A-MCF (10)(-3) & 18 & 88.3 & 0.5 \\
Prag (6)(-3) & 19 & 59.7 & 0.4 \\
\hline Not
\end{tabular}

\begin{tabular}{lrrr}
\hline \hline statut & rg. & p.c. & nor. \\
\hline Ponts et C. (7)(o) & 1 & 571.11 & 100.0 \\
DE ehess (13)(0) & 2 & 208.16 & 36.5 \\
DR cnrs (84)(0) & 3 & 66.60 & 11.7 \\
Insee (22)(0) & 4 & 60.98 & 10.7 \\
A-PR (97)(+3) & 5 & 39.66 & 6.9 \\
A-CR (21)(+4) & 6 & 35.64 & 6.2 \\
PR 05 (568)(-1) & 7 & 29.48 & 5.2 \\
DR inra (56)(-1) & 8 & 26.66 & $4 \cdot 7$ \\
DR cnrs non 37(10)(+10) & 9 & 25.33 & 4.4 \\
CR cnrs (102)(-5) & 10 & 22.56 & 4.0 \\
PR non 05(158)(+1) & 11 & 21.58 & 3.8 \\
Autre (13)(+5) & 12 & 19.21 & 3.4 \\
CR cnrs non 37 (9)(+5) & 13 & 17.54 & 3.1 \\
CR inra (89)(-5) & 14 & 14.26 & 2.5 \\
Prag (6)(-4) & 15 & 9.95 & 1.7 \\
A-MCF (10)(-3) & 16 & 9.19 & 1.6 \\
Insee non EC (27)(-2) & 17 & 8.97 & 1.6 \\
MCF 05 (1282)(-4) & 18 & 5.66 & 1.0 \\
MCF non 05 (260)(-3) & 19 & 4.16 & 0.7 \\
\hline
\end{tabular}

Notes : La colonne "rg." donne le rang, la colonne "tot." donne le score total, "nor." le score normalisé par rapport à celui du premier classé, "p.c." donne le score par chercheur. Entre les premières parenthèses se trouve le nombre de chercheurs, la variation de classement par rapport à $\mathrm{Clm}$ est donnée entre les deuxièmes parenthèses. Si cette variation est supérieure ou égale à 3, le nom est typographié en gras italique, si elle est inférieure ou égale à -3, le nom est typographié en italique, sinon le nom est simplement typographié en gras.

TABLE 17 - Statuts, Indice G, T=Dégressif

\begin{tabular}{lcrr}
\hline \hline statut & rg. & tot. & nor. \\
\hline PR 05 (568)(0) & 1 & 112.7 & 100.0 \\
A-PR (97)(+4) & 2 & 88.0 & 78.0 \\
DR cnrs (84)(o) & 3 & 85.1 & 75.5 \\
MCF o5 (1282)(-2) & 4 & 79.6 & 70.6 \\
PR non 05 (158)(+3) & 5 & 79.5 & 70.5 \\
Ponts et C. (7)(-1) & 6 & 75.0 & 66.5 \\
DE ehess (13)(+2) & 7 & 73.4 & 65.1 \\
Insee (22)(+4) & 8 & 62.2 & 55.2 \\
CR cnrs (102)(-5) & 9 & 56.9 & 50.5 \\
DR inra (56)(0) & 10 & 51.8 & 45.9 \\
CR inra (89)(-4) & 11 & 46.7 & 41.5 \\
A-CR (21)(+1) & 12 & 42.3 & 37.5 \\
MCF non 05 (260)(-2) & 13 & 33.6 & 29.8 \\
Insee non EC (27)(0) & 14 & 21.6 & 19.2 \\
Autre (13)(+2) & 15 & 21.3 & 18.9 \\
DR cnrs non 37 (10)(+3) & 16 & 18.1 & 16.0 \\
CR cnrs non 37 (9)(+1) & 17 & 12.8 & 11.4 \\
A-MCF (10)(-3) & 18 & 12.7 & 11.3 \\
Prag (6)(-3) & 19 & 9.9 & 8.8 \\
\hline Not
\end{tabular}

\begin{tabular}{lrrr}
\hline \hline statut & rg. & p.c. & nor. \\
\hline Ponts et C. (7)(o) & 1 & 13.90 & 100.0 \\
DE ehess (13)(0) & 2 & 12.53 & 90.2 \\
DR cnrs (84)(0) & 3 & 6.71 & 48.3 \\
Insee (22)(0) & 4 & 5.19 & 37.4 \\
DR inra (56)(+2) & 5 & 4.81 & 34.6 \\
DR cnrs non 37(10)(+13) & 6 & 4.33 & 31.2 \\
CR cnrs (102)(-2) & 7 & 4.13 & 29.7 \\
Autre (13)(+9) & 8 & 4.05 & 29.2 \\
PR 05 (568)(-3) & 9 & 3.72 & 26.8 \\
A-PR (97)(-2) & 10 & 3.55 & 25.6 \\
A-CR (21)(-1) & 11 & 3.02 & 21.7 \\
PR non 05(158)(0) & 12 & 2.95 & 21.2 \\
CR inra (89)(-4) & 13 & 2.75 & 19.8 \\
CR cnrs non 37 (9)(+4) & 14 & 2.64 & 19.0 \\
A-MCF (10)(-2) & 15 & 2.36 & 17.0 \\
Prag (6)(-5) & 16 & 2.09 & 15.0 \\
Insee non EC (27)(-2) & 17 & 1.70 & 12.2 \\
MCF 05 (1282)(-4) & 18 & 1.41 & 10.1 \\
MCF non 05 (260)(-3) & 19 & 1.19 & 8.6 \\
\hline
\end{tabular}

Notes : La colonne "rg." donne le rang, la colonne "tot." donne le score total, "nor." le score normalisé par rapport à celui du premier classé, "p.c." donne le score par chercheur. Entre les premières parenthèses se trouve le nombre de chercheurs, la variation de classement par rapport à $\mathrm{Clm}$ est donnée entre les deuxièmes parenthèses. Si cette variation est supérieure ou égale à 3, le nom est typographié en gras italique, si elle est inférieure ou égale à -3 , le nom est typographié en italique, sinon le nom est simplement typographié en gras. 
Le Tableau 18 corrige de la durée de la carrière. Les statuts apparaissant ci-dessus les plus productifs sont en effet aussi ceux pour lequel l'âge moyen est le plus élevé, ce qui a donné aux chercheurs plus de temps pour publier et donc pour recevoir des citations. Il apparaît que les différences observées entre statuts ne sont cependant pas dues à ce simple effet de l'âge, puisque la hiérarchie entre statuts est alors très proche de celle décrite précédemment.

TABLE 18 - Statuts, Citations totales, $\mathrm{T}=$ Carrière

\begin{tabular}{lrrr}
\hline \hline statut & rg. & tot. & nor. \\
\hline PR 05 (568)(o) & 1 & 3841.8 & 100.0 \\
MCF 05 (1282)(o) & 2 & 2014.7 & 52.4 \\
DR cnrs (84)(0) & 3 & 1425.3 & 37.1 \\
Ponts et C. (7)(+1) & 4 & 1286.3 & 33.5 \\
A-PR (97)(+1) & 5 & 923.1 & 24.0 \\
PR non 05 (158)(+3) & 6 & 874.0 & 22.8 \\
DE ehess (13)(+1) & 7 & 654.1 & 17.0 \\
CR cnrs (102)(-4) & 8 & 634.9 & 16.5 \\
CR inra (89)(-2) & 9 & 432.6 & 11.3 \\
Insee (22)(+1) & 10 & 398.4 & 10.4 \\
DR inra (56)(-1) & 11 & 324.7 & 8.5 \\
MCF non 05(260)(0) & 12 & 301.7 & 7.9 \\
A-CR (21)(0) & 13 & 170.4 & 4.4 \\
Autre (13)(+1) & 14 & 59.6 & 1.6 \\
DR cnrs non 37 (10)(+4) & 15 & 53.1 & 1.4 \\
CR cnrs non 37 (9)(+1) & 16 & 42.7 & 1.1 \\
Insee non EC (27)(-1) & 17 & 21.1 & 0.6 \\
A-MCF (10)(-4) & 18 & 16.3 & 0.4 \\
Prag (6)(-1) & 19 & 7.0 & 0.2 \\
\hline N
\end{tabular}

\begin{tabular}{lrrr}
\hline \hline statut & rg. & p.c. & nor. \\
\hline Ponts et C. (7)(o) & 1 & 172.66 & 100.0 \\
DE ehess (13)(0) & 2 & 51.30 & 29.7 \\
Insee (22)(+1) & 3 & 17.74 & 10.3 \\
DR cnrs (84)(-1) & 4 & 16.96 & 9.8 \\
A-PR (97)(+3) & 5 & 9.49 & 5.5 \\
A-CR (21)(+4) & 6 & 8.17 & 4.7 \\
PR 05 (568)(-1) & 7 & 6.76 & 3.9 \\
CR cnrs (102)(-3) & 8 & 6.23 & 3.6 \\
DR inra (56)(0) & 9 & 5.80 & 3.4 \\
PR non 05(158)(+2) & 10 & 5.54 & 3.2 \\
DR cnrs non 37 (10)(+8) & 11 & 5.31 & 3.1 \\
CR inra (89)(-5) & 12 & 4.86 & 2.8 \\
CR cnrs non 37 (9)(+2) & 13 & 4.74 & 2.8 \\
Autre (13)(+2) & 14 & 4.58 & 2.7 \\
A-MCF (10)(-4) & 15 & 1.70 & 1.0 \\
MCF 05 (1282)(-3) & 16 & 1.57 & 0.9 \\
Prag (6)(-3) & 17 & 1.17 & 0.7 \\
MCF non 05 (260)(-1) & 18 & 1.16 & 0.7 \\
Insee non EC (27)(-1) & 19 & 0.78 & 0.5 \\
\hline
\end{tabular}

Notes : La colonne "rg." donne le rang, la colonne "tot." donne le score total, "nor." le score normalisé par rapport à celui du premier classé, "p.c." donne le score par chercheur. Entre les premières parenthèses se trouve le nombre de chercheurs, la variation de classement par rapport à Clm est donnée entre les deuxièmes parenthèses. Si cette variation est supérieure ou égale à 3 , le nom est typographié en gras italique, si elle est inférieure ou égale à -3 , le nom est typographié en italique, sinon le nom est simplement typographié en gras.

Finalement, le Tableau 19 hiérarchise les statuts selon leur nombre de citations reçues par entrée GS. Il s'agit d'un point de vue relativement différent de celui des tableaux précédents qui correspond vraiment à une efficacité par article diffusé. Les statuts recevant beaucoup de citations globalement en reçoivent également beaucoup par entrée, mais on voit que là encore, les statuts qui ne sont pas au cœur de notre analyse progressent par rapport à Clm parfois encore plus que précédemment.

TABLE 19 - Statuts, Citations par entrée, T=Dégressif

\begin{tabular}{lcrr}
\hline \hline statut & rg. & tot. & nor. \\
\hline MCF 05 (1282)(+1) & 1 & 3108.3 & 100.0 \\
PR 05 (568)(-1) & 2 & 2599.8 & 83.6 \\
A-PR $(97)(+3)$ & 3 & 839.4 & 27.0 \\
PR non 05 (158)(+4) & 4 & 757.4 & 24.4 \\
DR cnrs (84)(-2) & 5 & 573.5 & 18.5 \\
MCF non 05 (260)(+5) & 6 & 573.2 & 18.4 \\
CR cnrs (102)(-3) & 7 & 377.6 & 12.2 \\
CR inra (89)(-1) & 8 & 315.8 & 10.2 \\
DR inra (56)(+1) & 9 & 241.0 & 7.8 \\
Insee (22)(+2) & 10 & 166.8 & 5.4 \\
Ponts et C. (7)(-6) & 11 & 159.0 & 5.1 \\
\hline
\end{tabular}

\begin{tabular}{|c|c|c|c|}
\hline statut & rg. & p.c. & nor. \\
\hline Ponts et C. $(7)(0)$ & 1 & 21.34 & 100.0 \\
\hline DR chrs non $37(10)(+17)$ & 2 & $14 \cdot 38$ & $67 \cdot 4$ \\
\hline DE ehess $(13)(-1)$ & 3 & 11.30 & 53.0 \\
\hline$A-P R(97)(+4)$ & 4 & 8.63 & 40.4 \\
\hline Insee $(22)(-1)$ & 5 & $7 \cdot 43$ & 34.8 \\
\hline$A-C R(21)(+4)$ & 6 & 7.15 & $33 \cdot 5$ \\
\hline DR cnrs $(84)(-4)$ & 7 & 6.82 & 32.0 \\
\hline$P R$ non o5 $(158)(+4)$ & 8 & 4.80 & 22.5 \\
\hline PR $05(568)(-3)$ & 9 & $4 \cdot 58$ & 21.4 \\
\hline$D R$ inra $(56)(-3)$ & 10 & $4 \cdot 30$ & 20.2 \\
\hline CR cnrs $(102)(-6)$ & 11 & 3.71 & $17 \cdot 4$ \\
\hline
\end{tabular}


suite de la page précédente

\begin{tabular}{lrrr}
\hline \hline statut & rg. & tot. & nor. \\
\hline A-CR (21)(+1) & 12 & 149.0 & 4.8 \\
DE ehess (13)(-4) & 13 & 144.1 & 4.6 \\
DR cnrs non 37 (10)(+5) & 14 & 143.8 & 4.6 \\
Insee non EC (27)(-1) & 15 & 87.6 & 2.8 \\
Autre (13)(+1) & 16 & 43.2 & 1.4 \\
A-MCF (10)(-2) & 17 & 32.5 & 1.0 \\
CR cnrs non 37 (9)(0) & 18 & 30.2 & 1.0 \\
Prag (6)(-3) & 19 & 14.1 & 0.5
\end{tabular}

\begin{tabular}{lrrr}
\hline \hline statut & rg. & p.c. & nor. \\
\hline CR inra (89)(-3) & 12 & 3.55 & 16.6 \\
A-MCF (10)(0) & 13 & 3.38 & 15.8 \\
CR cnrs non 37 (9)(+4) & 14 & 3.36 & 15.8 \\
Autre (13)(+2) & 15 & 3.32 & 15.6 \\
Insee non EC (27)(-1) & 16 & 3.24 & 15.2 \\
MCF O5 (1282)(-3) & 17 & 2.43 & 11.4 \\
Prag (6)(-7) & 18 & 2.35 & 11.0 \\
MCF non 05 (260)(-3) & 19 & 2.20 & 10.3 \\
\hline
\end{tabular}

Notes : La colonne "rg." donne le rang, la colonne "tot." donne le score total, "nor." le score normalisé par rapport à celui du premier classé, "p.c." donne le score par chercheur. Entre les premières parenthèses se trouve le nombre de chercheurs, la variation de classement par rapport à Clm est donnée entre les deuxièmes parenthèses. Si cette variation est supérieure ou égale à 3, le nom est typographié en gras italique, si elle est inférieure ou égale à -3 , le nom est typographié en italique, sinon le nom est simplement typographié en gras.

\section{Principaux classements}

Nous présentons dans cette section nos principaux classements. La période de temps retenue est $\mathrm{T}=$ Dégressif, ce qui représente un bon compromis avec la perspective de long terme, peut-être encore plus cruciale pour les indices de citations, celles-ci nécessitant nécessairement plus de temps pour se révéler, et une perspective de plus court-terme sûrement plus importante lorsque l'on se projette dans l'avenir.

Dans tous les tableaux de cette section (et de manière plus générale pour presque tous les tableaux de classements) la structure est la suivante. Le sous-tableau de gauche donne le classement en volume total, celui de droite le classement par chercheur. Dans chaque sous-tableau, les premières parenthèses après le nom de l'université/centre indiquent son nombre de chercheurs équivalent temps plein. Entre les deuxièmes parenthèses se trouve la variation du classement par rapport au classement $\mathrm{Clm}$. Si cette variation est supérieure ou égale à 3, le nom de l'université/centre est typographié en gras italique, si elle est inférieure ou égale à -3, le nom est typographié en italique, sinon le nom est simplement typographié en gras. La colonne "rg." donne le rang de l'université/centre, la colonne "tot." (resp. "p.c.") son score (resp. son score par chercheur), enfin, la colonne "nor." indique le score normalisé en \% du score de la meilleure université.

La Section 4.I rassemble quatre classements des universités. D'abord, un classement en volume et un par chercheur pour les citations totales, puis ces deux mêmes classements pour l'indice G. La section 4.2 présente les mêmes classements au niveau des centres de recherche.

Ces quatre classements apportent des éclairages différents. Tout d'abord, les classements en termes de volume mettent en avant les universités/centres dont la visibilité globale est la plus grande. Les classements en termes de scores par chercheur peuvent toutefois présenter des différences et ils permettent de mettre en avant des universités/centres avec un petit nombre de cher- 
cheurs très productifs mais dont la petite taille limite le rang dans les classements en volume. Certaines universités/centres sont bien classés selon les deux approches.

Nous ne re-détaillons pas les résultats obtenus dans toutes les sections de classements, chaque lecteur pouvant facilement chercher les éléments qui l'intéressent. Néanmoins, deux conclusions importantes semblent se dégager. D'une part, pour une majorité de centres ou universités, la variation de classements par rapport à Clm est faible. D'autre part, pour quelques autres, elles est relativement forte, et il s'avère que ces unités semblent être celles dont le cœur de l'activité n'est pas l'économie, ou, ce qui est lié, dont les membres ne sont pas au cœur de notre champ, à savoir les sections 5 du Cnu et 37 du Cnrs.

\subsection{Classements des universités}

\subsubsection{Citations totales}

TABLE 20 - Universités, Citations totales, T=Dégressif

\begin{tabular}{|c|c|c|c|}
\hline université & rg. & tot. & nor. \\
\hline Pse-Paris $1(214)(+1)$ & 1 & 9732.7 & 100.0 \\
\hline Tse-Toulouse $\mathbf{I}(125)(-1)$ & 2 & 9550.6 & 98.1 \\
\hline Crest-Ensae $(67)(+1)$ & 3 & 3487.8 & 35.8 \\
\hline Hec $(75)(+1)$ & 4 & 2874.1 & 29.5 \\
\hline Aix Marseille 2-3 (115)(-2) & 5 & 2250.1 & 23.1 \\
\hline Paris $9(124)(+6)$ & 6 & 1553.0 & 16.0 \\
\hline Nancy 2-Strasbourg 1 (95)(o) & 7 & 1292.0 & $13 \cdot 3$ \\
\hline Ec. Polytechnique $(34)(-2)$ & 8 & 1248.4 & 12.8 \\
\hline Paris 10 $(80)(-1)$ & 9 & 1212.9 & 12.5 \\
\hline Grenoble 2-Inra $(128)(+4)$ & 10 & 980.2 & 10.1 \\
\hline Lille 1-Polytech Lille (153)(-1) & 11 & 963.9 & 9.9 \\
\hline Montpellier 1-Inra $(62)(+1)$ & 12 & 914.5 & $9 \cdot 4$ \\
\hline Lyon $2(70)(+3)$ & 13 & 877.8 & 9.0 \\
\hline Iep Paris $(9)(+5)$ & 14 & 860.3 & 8.8 \\
\hline Cergy Pontoise (37)(-6) & 15 & 789.0 & 8.1 \\
\hline Dijon $(65)(+14)$ & 16 & 750.2 & $7 \cdot 7$ \\
\hline Bordeaux $4(72)(-2)$ & 17 & $717 \cdot 4$ & $7 \cdot 4$ \\
\hline Nice $(83)(-1)$ & 18 & $697 \cdot 7$ & 7.2 \\
\hline Caen-Rennes 1 (121)(-8) & 19 & 696.5 & 7.2 \\
\hline Clermont $1(32)(+3)$ & 20 & 536.4 & $5 \cdot 5$ \\
\hline Paris $11(36)(+21)$ & 21 & 525.9 & $5 \cdot 4$ \\
\hline Paris $13(45)(+3)$ & 22 & 520.0 & $5 \cdot 3$ \\
\hline Inra Vers-Grig $(12)(+1)$ & 23 & $517 \cdot 4$ & $5 \cdot 3$ \\
\hline Paris $2(41)(-6)$ & 24 & 457.2 & 4.7 \\
\hline Cired $(14)(+7)$ & 25 & 451.1 & 4.6 \\
\hline $\operatorname{Besancon}^{(24)(-4)}$ & 26 & 402.3 & 4.1 \\
\hline Inra Ivry $(37)(-1)$ & 27 & 347.2 & 3.6 \\
\hline Inra Rennes (12)(-1) & 28 & 343.1 & 3.5 \\
\hline Versailles St Quentin (24)(+12) & 29 & 319.6 & $3 \cdot 3$ \\
\hline Nantes $(23)(-9)$ & 30 & 288.3 & 3.0 \\
\hline Strasbourg $3(13)(-2)$ & 31 & 281.9 & 2.9 \\
\hline Ens Cachan $(7)(+1)$ & 32 & 251.7 & 2.6 \\
\hline Orleans $(34)(-5)$ & 33 & 234.5 & 2.4 \\
\hline Lille $2(13)(+12)$ & 34 & 233.5 & 2.4 \\
\hline Chambery $(15)(+16)$ & 35 & 206.9 & 2.1 \\
\hline Le Mans $(18)(-16)$ & 36 & 204.4 & 2.1 \\
\hline $\operatorname{Pau}(17)(+7)$ & 37 & 202.3 & 2.1 \\
\hline Reims $(32)(-3)$ & 38 & $197 \cdot 5$ & 2.0 \\
\hline Paris $8(27)(-5)$ & 39 & 185.5 & 1.9 \\
\hline Evry (18)(-9) & 40 & 169.3 & 1.7 \\
\hline St Etienne $(22)(-3)$ & 41 & 145.4 & 1.5 \\
\hline Paris $12(30)(+13)$ & 42 & 139.6 & 1.4 \\
\hline
\end{tabular}

\begin{tabular}{|c|c|c|c|}
\hline université & rg. & p.c. & nor. \\
\hline Iep Paris $(9)(+1)$ & 1 & 101.21 & 100.0 \\
\hline Tse-Toulouse 1 (125)(-1) & 2 & 76.65 & 75.7 \\
\hline Crest-Ensae $(67)(+1)$ & 3 & 51.98 & 51.4 \\
\hline Pse-Paris $1(214)(+1)$ & 4 & $45 \cdot 50$ & 45.0 \\
\hline Inra Vers-Grig $(12)(+2)$ & 5 & 43.11 & 42.6 \\
\hline Hec $(75)(+5)$ & 6 & 38.37 & 37.9 \\
\hline Ec. Polytechnique (34)(-4) & 7 & 36.56 & 36.1 \\
\hline Ens Cachan $(7)(-2)$ & 8 & $34 \cdot 33$ & 33.9 \\
\hline Cired $(14)(+6)$ & 9 & 33.41 & 33.0 \\
\hline Inra Rennes (12)(-1) & 10 & 28.59 & 28.3 \\
\hline Strasbourg $3(13)(+3)$ & 11 & 22.02 & 21.8 \\
\hline Cergy Pontoise (37) $(-4)$ & 12 & 21.32 & 21.1 \\
\hline Aix Marseille 2-3 (115)(-1) & 13 & 19.59 & 19.4 \\
\hline Lille $2(13)(+17)$ & 14 & 17.96 & 17.8 \\
\hline $\operatorname{Besancon}(24)(+3)$ & 15 & 16.76 & 16.6 \\
\hline ont $1(32)(+10)$ & 16 & 16.76 & 16.6 \\
\hline Paris $10(80)(+3)$ & 17 & 15.15 & 15.0 \\
\hline Montpellier 1-Inra $(62)(+8)$ & 18 & 14.75 & 14.6 \\
\hline Paris $11(36)(+47)$ & 19 & 14.61 & 14.4 \\
\hline La Rochelle (5)(-7) & 20 & 13.98 & 13.8 \\
\hline Chambery $(15)(+26)$ & 21 & 13.79 & 13.6 \\
\hline Nancy 2-Strasbourg I (95)(o) & 22 & 13.60 & 13.4 \\
\hline Versailles St Quentin $(24)(+32)$ & 23 & 13.32 & 13.2 \\
\hline Inra Nancy $(7)(+17)$ & 24 & 12.92 & 12.8 \\
\hline Nantes $(23)(-8)$ & 25 & 12.81 & 12.7 \\
\hline Paris $9(124)(+22)$ & 26 & 12.48 & 12.3 \\
\hline $2(70)(+5)$ & 27 & 12.47 & 12.3 \\
\hline Pau $(17)(+14)$ & 28 & 12.26 & 12.1 \\
\hline Dijon $(65)(+36)$ & 29 & 11.56 & 11.4 \\
\hline Paris $13(45)(+11)$ & 30 & 11.56 & 11.4 \\
\hline Paris $7(10)(+37)$ & 31 & 11.47 & 11.3 \\
\hline Inra Dijon (11)(-16) & 32 & 11.45 & 11.3 \\
\hline Paris $2(41)(-9)$ & 33 & 11.29 & 11.2 \\
\hline Le Mans $(18)(-24)$ & $\begin{array}{l}35 \\
34\end{array}$ & 11.20 & 11.1 \\
\hline Bordeaux $4(72)(-1)$ & 35 & 10.03 & 9.9 \\
\hline Evry (18)(-15) & 36 & 9.41 & $9 \cdot 3$ \\
\hline Inra Ivry $(37)(-1)$ & 37 & 9.38 & $9 \cdot 3$ \\
\hline Nice $(83)(+14)$ & 38 & 8.46 & 8.4 \\
\hline Lille $3(11)(-16)$ & 39 & 8.03 & $7 \cdot 9$ \\
\hline Rennes $2(7)(+11)$ & 40 & $7 \cdot 73$ & 7.6 \\
\hline Grenoble 2-Inra $(128)(+19)$ & 41 & 7.66 & 7.6 \\
\hline Montpellier $3(10)(-14)$ & 42 & 7.43 & $7 \cdot 4$ \\
\hline
\end{tabular}


suite de la page précédente

\begin{tabular}{|c|c|c|c|}
\hline université & rg. & tot. & nor. \\
\hline Inra Dijon (11)(-7) & 43 & 125.9 & 1.3 \\
\hline Paris $7(10)(+26)$ & 44 & 114.7 & 1.2 \\
\hline Rouen $(22)(+19)$ & 45 & 106.9 & 1.1 \\
\hline Limoges $(18)(-1)$ & 46 & 96.5 & 1.0 \\
\hline Lille $3(11)(-4)$ & 47 & 88.4 & 0.9 \\
\hline La Reunion (19)(-9) & 48 & $87 \cdot 4$ & 0.9 \\
\hline Perpignan (12)(-12) & 49 & $85 \cdot 3$ & 0.9 \\
\hline Inra Nancy (7)(+15) & 50 & 84.0 & 0.9 \\
\hline Angers $(18)(+8)$ & 51 & $73 \cdot 3$ & 0.8 \\
\hline Montpellier $3(10)(+2)$ & 52 & 70.6 & 0.7 \\
\hline La Rochelle (5)(o) & 53 & 69.9 & 0.7 \\
\hline Toulon $(11)(+3)$ & 54 & 63.2 & 0.7 \\
\hline Poitiers $(27)(-15)$ & 55 & 61.3 & 0.6 \\
\hline Tours (12)(-9) & 56 & 59.2 & 0.6 \\
\hline Littoral (13)(-9) & 57 & 54.2 & 0.6 \\
\hline Rennes $2(7)(+9)$ & 58 & 54.1 & 0.6 \\
\hline Marne La Vallee (11)(-7) & 59 & $53 \cdot 3$ & 0.6 \\
\hline Mulhouse $(9)(-4)$ & 60 & 52.4 & 0.5 \\
\hline Toulouse $2(10)(+14)$ & 61 & 52.2 & 0.5 \\
\hline Brest $(21)(-13)$ & 62 & 51.2 & 0.5 \\
\hline Antilles Guyane (22)(-13) & 63 & 50.5 & 0.5 \\
\hline Valenciennes $(8)(+5)$ & 64 & 48.0 & 0.5 \\
\hline Le Havre $(15)(+1)$ & 65 & 42.5 & 0.4 \\
\hline Cnam $(7)(-5)$ & 66 & 42.4 & 0.4 \\
\hline Artois (13) (-7) & 67 & 39.8 & 0.4 \\
\hline Metz $(13)(-5)$ & 68 & 38.6 & 0.4 \\
\hline Paris 5 (11)(-11) & 69 & 33.1 & 0.3 \\
\hline Amiens $(22)(-8)$ & 70 & 29.7 & 0.3 \\
\hline Toulouse $3(10)(+2)$ & 71 & $27 \cdot 7$ & 0.3 \\
\hline Lyon $1(5)(-1)$ & 72 & 24.5 & 0.3 \\
\hline Corte $(9)(-1)$ & 73 & 15.9 & 0.2 \\
\hline Bretagne Sud (9)(-6) & 74 & $13 \cdot 3$ & 0.1 \\
\hline Lyon $3(9)(-1)$ & 75 & 8.1 & 0.1 \\
\hline
\end{tabular}

\begin{tabular}{|c|c|c|c|}
\hline université & rg. & p.c. & nor. \\
\hline Perpignan $(12)(-24)$ & 43 & $7 \cdot 41$ & \\
\hline Orleans $(34)(-7)$ & 44 & 6.90 & 6.8 \\
\hline Paris $8(27)(-7)$ & 45 & 6.87 & 6.8 \\
\hline St Etienne $(22)(-2)$ & 46 & 6.61 & 6.5 \\
\hline Valenciennes $(8)(+14)$ & 47 & 6.40 & 6.3 \\
\hline Lille 1-Polytech Lille $(153)(+6)$ & 48 & 6.29 & 6.2 \\
\hline Reims $(32)(+1)$ & 49 & 6.27 & 6.2 \\
\hline Mulhouse (9)(-23) & 50 & 6.16 & 6.1 \\
\hline $\operatorname{Cnam}(7)(-16)$ & 51 & 6.05 & 6.0 \\
\hline Caen-Rennes 1 (121)(-9) & 52 & $5 \cdot 76$ & $5 \cdot 7$ \\
\hline Toulon $(11)(-7)$ & 53 & $5 \cdot 75$ & 5.7 \\
\hline Toulouse $2(10)(+21)$ & 54 & 5.49 & $5 \cdot 4$ \\
\hline Limoges (18)(-6) & 55 & $5 \cdot 36$ & $5 \cdot 3$ \\
\hline Marne La Vallee (11)(-27) & 56 & 5.08 & 5.0 \\
\hline Tours $(12)(-27)$ & 57 & 4.93 & 4.9 \\
\hline Lyon $1(5)(-5)$ & 58 & 4.91 & 4.9 \\
\hline Rouen $(22)(+12)$ & 59 & 4.86 & 4.8 \\
\hline Paris $12(30)(+9)$ & 60 & 4.73 & 4.7 \\
\hline La Reunion (19)(-22) & 61 & 4.60 & 4.6 \\
\hline Littoral (13)(-29) & 62 & 4.17 & 4.1 \\
\hline Angers (18)(+1) & 63 & 4.07 & 4.0 \\
\hline Artois $(13)(-6)$ & 64 & 3.19 & 3.2 \\
\hline Paris $5(11)(-20)$ & 65 & 3.16 & 3.1 \\
\hline $\operatorname{Metz}(13)(-4)$ & 66 & 3.09 & 3.1 \\
\hline Le Havre (15)(o) & 67 & 2.83 & 2.8 \\
\hline Toulouse $3(10)(+5)$ & 68 & 2.77 & 2.7 \\
\hline Brest $(21)(-13)$ & 69 & 2.50 & 2.5 \\
\hline Antilles Guyane (22)(-11) & 70 & 2.35 & 2.3 \\
\hline Poitiers $(27)(-14)$ & 71 & 2.27 & 2.2 \\
\hline Corte $(9)(0)$ & 72 & 1.77 & 1.8 \\
\hline Bretagne Sud (9)(-10) & 73 & 1.48 & 1.5 \\
\hline Amiens (22)(-4) & 74 & 1.35 & 1.3 \\
\hline Lyon $3(9)(-1)$ & 75 & 0.90 & 0.9 \\
\hline
\end{tabular}

Notes : La colonne "rg." donne le rang, la colonne "tot." donne le score total, "nor." le score normalisé par rapport à celui du premier classé, "p.c." donne le score par chercheur. Entre les premières parenthèses se trouve le nombre de chercheurs, la variation de classement par rapport à Clm est donnée entre les deuxièmes parenthèses. Si cette variation est supérieure ou égale à 3 , le nom est typographié en gras italique, si elle est inférieure ou égale à -3 , le nom est typographié en italique, sinon le nom est simplement typographié en gras.

\subsubsection{Indice G}

TABLE 21 - Universités, Indice G, T=Dégressif

\begin{tabular}{lcrr}
\hline \hline université & rg. & tot. & nor. \\
\hline Pse-Paris 1 (214)(+1) & 1 & 112.0 & 100.0 \\
Tse-Toulouse 1 (125)(-1) & 2 & 104.6 & 93.4 \\
Hec (75)(+2) & 3 & 72.7 & 64.9 \\
Crest-Ensae (67)(0) & 4 & 64.2 & 57.4 \\
Aix Marseille 2-3 (115)(-2) & 5 & 63.4 & 56.6 \\
Nancy 2-Strasbourg 1 (95)(+1) & 6 & 52.2 & 46.7 \\
Paris 9 (124)(+5) & 7 & 47.2 & 42.2 \\
Ec. Polytechnique (34)(-2) & 8 & 45.5 & 40.6 \\
Paris 10 (80)(-1) & 9 & 45.1 & 40.3 \\
Iep Paris (9)(+9) & 10 & 43.6 & 38.9 \\
Montpellier 1-Inra (62)(+2) & 11 & 43.1 & 38.5 \\
Lyon 2 (70)(+4) & 12 & 40.4 & 36.1 \\
Grenoble 2-Inra (128)(+1) & 13 & 36.4 & 32.5 \\
Lille 1-Polytech Lille (153)(-3) & 14 & 36.4 & 32.5 \\
Paris 2 (41)(+3) & 15 & 36.0 & 32.2 \\
Nice (83)(+1) & 16 & 35.0 & 31.2 \\
Inra Vers-Grig (12)(+7) & 17 & 34.0 & 30.4 \\
\hline
\end{tabular}

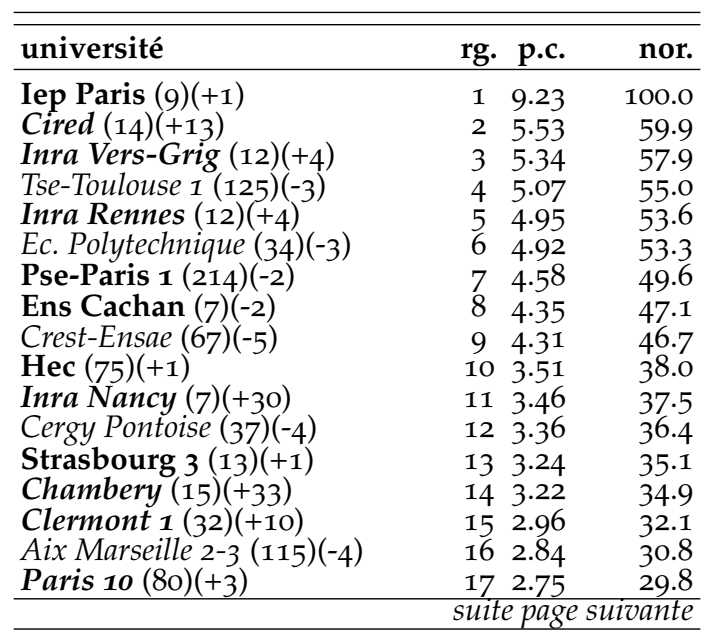


suite de la page précédente

\begin{tabular}{|c|c|c|c|}
\hline université & rg. & tot. & nor. \\
\hline Dijon $(65)(+12)$ & 18 & 32.4 & 29.0 \\
\hline Caen-Rennes 1 (121)(-8) & 19 & 32.2 & 28.8 \\
\hline Cergy Pontoise $(37)(-11)$ & 20 & 32.1 & 28.7 \\
\hline Le Mans (18)(-1) & 21 & 31.0 & $27 \cdot 7$ \\
\hline Cired $(14)(+10)$ & 22 & 30.7 & $27 \cdot 5$ \\
\hline Clermont $1(32)(0)$ & 23 & 30.0 & 26.8 \\
\hline Bordeaux $4(72)(-9)$ & 24 & 29.7 & 26.5 \\
\hline Paris $11(36)(+17)$ & 25 & 29.4 & 26.3 \\
\hline Strasbourg $3(13)(+3)$ & 26 & 27.2 & 24.3 \\
\hline Inra Rennes (12)(o) & 27 & 26.2 & $23 \cdot 4$ \\
\hline Ens Cachan $(7)(+5)$ & 28 & 25.8 & 23.0 \\
\hline$(45)(-4)$ & 29 & 24.6 & 22.0 \\
\hline Besancon $(24)(-8)$ & 30 & $24 \cdot 5$ & 21.9 \\
\hline Inra Ivry $(37)(-5)$ & 31 & $24 \cdot 4$ & 21.8 \\
\hline Versailles St Quentin (24)(+9) & 32 & 24.0 & 21.5 \\
\hline Lille $2(13)(+13)$ & 33 & 23.7 & 21.2 \\
\hline Chambery $(15)(+17)$ & 34 & 22.8 & 20.3 \\
\hline Nantes $(23)(-14)$ & 35 & 22.2 & 19.9 \\
\hline+8$)^{14}$ & 36 & 20.9 & 18.6 \\
\hline$(32)(-2)$ & 37 & 20.3 & 18.1 \\
\hline 8$)(-7)$ & 38 & 18.7 & 16.7 \\
\hline 34$)(-11)$ & 39 & 18.2 & 16.2 \\
\hline$(18)(+5)$ & 40 & 17.2 & $15 \cdot 4$ \\
\hline Sijon $(11)(-5)$ & 41 & 17.1 & $15 \cdot 3$ \\
\hline $7(10)(+28)$ & 42 & 16.3 & 14.6 \\
\hline$(22)(-5)$ & 43 & 15.9 & 14.2 \\
\hline 30$)(+11)$ & 44 & $15 \cdot 3$ & 13.7 \\
\hline 3) $(+14)$ & 45 & 15.2 & 13.6 \\
\hline$(-3)$ & 46 & 14.4 & 12.9 \\
\hline Inra Nancy $(7)(+18)$ & 47 & 13.8 & 12.3 \\
\hline$(27)(-14)$ & 48 & 13.5 & 12.0 \\
\hline$(+15)$ & 49 & 13.2 & 11.8 \\
\hline ion $(19)(-11)$ & 50 & 13.1 & 11.7 \\
\hline nnes $(8)(+18)$ & 51 & 13.0 & 11.7 \\
\hline lier $3(10)(-$ & 52 & 12.9 & 11.5 \\
\hline$(12)(-16)$ & 53 & 12.5 & 11.1 \\
\hline I) $(+3)$ & 54 & 12.2 & 10.9 \\
\hline$(9)(+1)$ & 55 & 11.9 & 10.7 \\
\hline 7) $(-16)$ & 56 & 11.8 & 10.5 r \\
\hline$(-10)$ & 57 & 11.7 & 10.5 \\
\hline$(3)(+2)$ & 58 & 11.1 & 9.9 \\
\hline$(-9)$ & 59 & 11.1 & 9.9 \\
\hline Vallee $(11)(-8)$ & 60 & 10.4 & $9 \cdot 3$ \\
\hline $2(10)(+15)$ & 61 & 10.4 & $9 \cdot 3$ \\
\hline 15$)(+4)$ & 62 & 10.1 & $\begin{array}{l}9.3 \\
9.0\end{array}$ \\
\hline (o) & 63 & 9.7 & 8.7 \\
\hline uyane $(22)(-14)$ & 64 & 9.6 & 8.6 \\
\hline & 65 & 9.4 & 8.4 \\
\hline$l e(5)(-13)$ & 66 & $9 \cdot 3$ & 8.3 \\
\hline$(7)(+1)$ & 67 & 9.3 & 8.3 \\
\hline 1) $(-10)$ & 68 & 8.1 & 7.2 \\
\hline 3) $(-21)$ & 69 & 7.6 & 6.8 \\
\hline se $3(10)(+3)$ & 70 & 5.9 & 5.2 \\
\hline & 71 & 5.2 & 4.7 \\
\hline $3(9)(+3)$ & 72 & 5.2 & 4 . \\
\hline $1(5)(-2)$ & 73 & 4.6 & 4.1 \\
\hline Amiens (22)(-12) & 74 & $4 \cdot 5$ & \\
\hline Bretagne Sud (9)(-7) & 75 & 4.2 & \\
\hline
\end{tabular}

\begin{tabular}{|c|c|c|c|}
\hline université & rg. & p.c. & nor. \\
\hline Paris $13(45)(+22)$ & 18 & 2.73 & 29.6 \\
\hline Paris $11(36)(+47)$ & 19 & 2.65 & 28.7 \\
\hline La Rochelle $(5)(-7)$ & 20 & 2.48 & 26.9 \\
\hline Perpignan $(12)(-2)$ & 21 & 2.43 & 26.3 \\
\hline Besancon $(24)(-4)$ & 22 & 2.39 & 25.8 \\
\hline Nancy 2-Strasbourg 1 (95)(-1) & 23 & 2.37 & 25.6 \\
\hline $\operatorname{Lyon} 2(70)(+8)$ & 24 & 2.36 & 25.5 \\
\hline Versailles St Quentin $(24)(+31)$ & 25 & 2.36 & 25.6 \\
\hline Le Mans (18)(-16) & 26 & 2.33 & $25 \cdot 3$ \\
\hline Montpellier $3(10)(+1)$ & 27 & 2.32 & 25.2 \\
\hline Nantes (23)(-11) & 28 & 2.30 & 24.9 \\
\hline Rennes $2(7)(+22)$ & 29 & 2.27 & 24.6 \\
\hline Evry (18)(-9) & 30 & 2.21 & 23.9 \\
\hline Inra Dijon $(11)(-15)$ & 31 & 2.20 & 23.8 \\
\hline Montpellier 1-Inra (62)(-6) & 32 & 2.17 & 23.5 \\
\hline Pau $(17)(+10)$ & 33 & 2.17 & 23.5 \\
\hline Dijon $(65)(+31)$ & 34 & 2.15 & $23 \cdot 3$ \\
\hline Lille $3(11)(-12)$ & 35 & 2.12 & 23.0 \\
\hline Bordeaux $4(72)(-2)$ & 36 & 2.03 & 22.0 \\
\hline Inra Ivry $(37)(-1)$ & 37 & 2.01 & 21.7 \\
\hline Paris $9(124)(+11)$ & 38 & 2.01 & 21.8 \\
\hline $\operatorname{Cnam}(7)(-4)$ & 39 & 1.92 & 20.8 \\
\hline Grenoble 2-Inra $(128)(+20)$ & 40 & 1.84 & 19.9 \\
\hline Nice $(83)(+11)$ & 41 & 1.82 & 19.7 \\
\hline Paris $2(41)(-18)$ & 42 & 1.78 & $19 \cdot 3$ \\
\hline Paris $7(10)(+25)$ & 43 & 1.74 & 18.8 \\
\hline St Etienne (22)(o) & 44 & 1.73 & 18.7 \\
\hline Caen-Rennes $1(121)(-2)$ & 45 & 1.72 & 18.7 \\
\hline Reims $(32)(+4)$ & 46 & 1.68 & 18.2 \\
\hline Lille $2(13)(-16)$ & 47 & 1.67 & 18.1 \\
\hline Orleans $(34)(-11)$ & 48 & 1.64 & 17.8 \\
\hline Paris $8(27)(-11)$ & 49 & 1.62 & $17 \cdot 5$ \\
\hline Toulon (11) $(-3)$ & 50 & 1.62 & 17.5 \\
\hline Marne La Vallee (11)(-22) & 51 & 1.56 & 16.9 \\
\hline Lille 1-Polytech Lille $(153)(+2)$ & 52 & 1.51 & 16.3 \\
\hline Mulhouse (9)(-26) & 53 & 1.49 & 16.2 \\
\hline Toulouse $2(10)(+22)$ & 54 & 1.49 & 16.2 \\
\hline$t(22)(+16)$ & 55 & 1.42 & 15.4 \\
\hline La Reunion (19)(-17) & 56 & 1.36 & 14.8 \\
\hline Littoral $(13)(-24)$ & 57 & 1.34 & 14.5 \\
\hline $2(30)(+11)$ & 58 & 1.29 & 14.0 \\
\hline Le Havre $(15)(+8)$ & 59 & 1.28 & 13.8 \\
\hline Tours $(12)(-30)$ & 60 & 1.21 & 13.1 \\
\hline es $(18)(-12)$ & 61 & 1.19 & 12.8 \\
\hline Toulouse 3 (10)(+11) & 62 & 1.01 & 10.9 \\
\hline Valenciennes (8)(-1) & 63 & 1.01 & 11.0 \\
\hline Paris $5(11)(-19)$ & 64 & 0.93 & 10.1 \\
\hline Lyon $1(5)(-12)$ & 65 & 0.92 & 9.9 \\
\hline $\operatorname{Metz}(13)(-4)$ & 66 & 0.88 & $9 \cdot 5$ \\
\hline Poitiers $(27)(-9)$ & 67 & 0.88 & 9.5 \\
\hline Brest $(21)(-12)$ & 68 & 0.83 & 9.0 \\
\hline Corte $(9)(+4)$ & 69 & 0.83 & 9.0 \\
\hline Antilles Guyane (22)(-11) & 70 & 0.81 & 8.8 \\
\hline Angers (18)(-7) & 71 & 0.78 & 8.5 \\
\hline Bretagne Sud (9)(-9) & 72 & 0.77 & 8.3 \\
\hline Artois (13)(-15) & 73 & 0.75 & 8.1 \\
\hline Amiens $(22)(-4)$ & 74 & 0.63 & 6.8 \\
\hline Lyon $3(9)(-1)$ & 75 & 0.45 & 4.9 \\
\hline
\end{tabular}

Notes : La colonne "rg." donne le rang, la colonne "tot." donne le score total, "nor." le score normalisé par rapport à celui du premier classé, "p.c." donne le score par chercheur. Entre les premières parenthèses se trouve le nombre de chercheurs, la variation de classement par rapport à Clm est donnée entre les deuxièmes parenthèses. Si cette variation est supérieure ou égale à 3 , le nom est typographié en gras italique, si elle est inférieure ou égale à -3, le nom est typographié en italique, sinon le nom est simplement typographié en gras. 


\subsection{Classements des centres}

TABLE 22 - Centres en 2008, Citations totales, T=Dégressif

\begin{tabular}{|c|c|c|c|}
\hline centre & rg. & tot. & nor. \\
\hline Gremaq (Tse-Toulouse 1) (59)(o) & 1 & $7575 \cdot 5$ & 100.0 \\
\hline Pjse (Pse-Paris 1) (43)(+1) & 2 & 5530.9 & 73.0 \\
\hline Ces (Pse-Paris 1$)(138)(-1)$ & 3 & 4010.3 & 52.9 \\
\hline Greghec $(\mathrm{Hec})(75)(+1)$ & 4 & 2874.1 & 37.9 \\
\hline Greqam (Aix Marseille 2-3) (45)(-1) & 5 & 1920.2 & $25 \cdot 4$ \\
\hline Non-Grecsta (Crest-Ensae) $(36)(+6)$ & 6 & 1777.0 & 23.5 \\
\hline Grecsta (Crest-Ensae) (31) $(-1)$ & 7 & 1710.8 & 22.6 \\
\hline Lerna (Tse-Toulouse 1) (19)(-1) & 8 & 1296.1 & 17.1 \\
\hline Preg (Ec. Polytechnique) (34)(-1) & 9 & 1248.4 & 16.5 \\
\hline Beta (Nancy 2-Strasb. 1) (72)(o) & 10 & 1214.9 & 16.0 \\
\hline Economix (Paris 10) $(66)(-2)$ & 11 & 1186.5 & 15.7 \\
\hline Drm (Paris 9) (69)(+15) & 12 & 1093.7 & 14.4 \\
\hline Centre (Iep Paris) $(9)(+5)$ & 13 & 860.3 & 11.4 \\
\hline Thema (Cergy Pontoise) (36)(-3) & 14 & 787.8 & 10.4 \\
\hline Lameta (Montpellier 1-Inra) $(33)(+1)$ & 15 & 737.2 & 9.7 \\
\hline Vice) $(71)(+1)$ & 16 & $634 \cdot 3$ & \\
\hline Crem (Caen-Rennes 1) (82)(-4) & 17 & 588.2 & 7.8 \\
\hline Cerdi (Clermont 1) (26) $(+7)$ & 18 & 536.4 & 7.1 \\
\hline Gate (Lyon 2) (27) $(-4)$ & 19 & 534.9 & 7.1 \\
\hline Eco. Pub. (Inra Vers-Grig) $(12)(+6)$ & 20 & $517 \cdot 4$ & 6.8 \\
\hline $\operatorname{Leg}($ Dijon $)(42)(+26)$ & 21 & 478.8 & 6.3 \\
\hline Non-Drm (Paris 9) (56)(-8) & 22 & $459 \cdot 3$ & 6.1 \\
\hline Centre (Cired) (14) $(+14)$ & 23 & 451.1 & 6.0 \\
\hline$($ Paris 13$)(33)(+7)$ & 24 & 444.6 & 5.9 \\
\hline Lem (Lille 1-Poly. Lille) $(87)(-2)$ & 25 & $435 \cdot 5$ & 5.8 \\
\hline$a($ Bordeaux 4) $(37)(-6)$ & 26 & 434.4 & $5 \cdot 7$ \\
\hline 4) $(-3)$ & 27 & 402.3 & $5 \cdot 3$ \\
\hline ouse 1) $(23)(-7)$ & 28 & 375.2 & 5.0 \\
\hline Cerag (Grenoble 2-Inra) $(48)(+25)$ & 29 & $355 \cdot 5$ & 4.7 \\
\hline e-Eco (Lille 1-Poly. Lille) $(36)(+4)$ & 30 & 352.4 & 4.7 \\
\hline t (Inra Rennes) $(12)(-1)$ & 31 & 343.1 & 4.5 \\
\hline Non-Gate (Lyon 2) $(43)(+14)$ & 32 & 342.9 & 4.5 \\
\hline uentin) $(24)(+20)$ & 33 & 319.6 & 4.2 \\
\hline$(17)(-1)$ & 34 & 298.3 & 3.9 \\
\hline$(-13)$ & 35 & 288.3 & 3.8 \\
\hline retha (Bordeaux 4) $(35)(+4)$ & 36 & 283.0 & 3.7 \\
\hline 3$)(-2)$ & 37 & 281.9 & 3.7 \\
\hline 5) $(+4)$ & 38 & 273.5 & 3.6 \\
\hline Non-Cermes (Paris 11) $(16)(+24)$ & 39 & 272.6 & 3.6 \\
\hline-59$)$ & 40 & $253 \cdot 3$ & $3 \cdot 3$ \\
\hline e-Toulouse 1) $(20)(+44)$ & 41 & 252.5 & $3 \cdot 3$ \\
\hline Cachan) $(7)(-4)$ & 42 & 251.7 & $3 \cdot 3$ \\
\hline$(+17)^{4}$ & 43 & $233 \cdot 5$ & 3.1 \\
\hline$(30)(-12)$ & 44 & 231.6 & 3.1 \\
\hline ble $2-$ Inra) $(29)(+29)$ & 45 & 231.0 & 3.1 \\
\hline & 46 & 212.5 & 2.8 \\
\hline$(15)(+19)$ & 47 & 206.9 & 2.7 \\
\hline (Le Mans) (18)(-29) & 48 & 204.4 & 2.7 \\
\hline$(\mathrm{Pau})(17)(+7)$ & 49 & 202.3 & 2.7 \\
\hline Marseille 2-3) (57)(+7) & 50 & 202.2 & 2.7 \\
\hline$(32)(-7)$ & 51 & $197 \cdot 5$ & 2.6 \\
\hline $\begin{array}{ll}5 & 1) \\
(33)(+16)\end{array}$ & 52 & 191.6 & 2.5 \\
\hline 8) $(27)(-14)$ & 53 & 185.5 & 2.5 \\
\hline$(19)(-25)$ & 54 & 177.0 & 2.3 \\
\hline oly. Lille) $(31)(-27)$ & 55 & 176.1 & 2.3 \\
\hline -Tsv (Inra Ivry) (19) $(+45)$ & 56 & 170.2 & 2.3 \\
\hline Centre (Evry) (18)(-21) & 57 & 169.3 & 2.2 \\
\hline Non-Ermes (Paris 2) (24)(-17) & 58 & 158.9 & 2.1 \\
\hline Centre (St Etienne) $(22)(-9)$ & 59 & 145.4 & 1.9 \\
\hline$(30)(+11)$ & 60 & 139.6 & 1.8 \\
\hline Lest-Eco (Aix Marseille 2-3) (13)(+29) & 61 & 127.7 & 1.7 \\
\hline Cesaer (Inra Dijon) (11)(-17) & 62 & 125.9 & 1.7 \\
\hline ble 2-Inra) $(38)(-20)$ & 63 & 120.2 & 1.6 \\
\hline Centre (Paris 7$)(10)(+31)$ & 64 & 114.7 & 1.5 \\
\hline
\end{tabular}

\begin{tabular}{|c|c|c|c|}
\hline centre & rg. & p.c. & nor. \\
\hline Pjse (Pse-Paris 1) (43)(+1) & 1 & 128.18 & 100.0 \\
\hline Gremaq (Tse-Toulouse 1) (59)(-1) & 2 & 127.96 & 99.8 \\
\hline Centre (Iep Paris) $(9)(+2)$ & 3 & 101.21 & 79.0 \\
\hline Lerna (Tse-Toulouse 1) (19)(-1) & 4 & 70.06 & 54.7 \\
\hline Grecsta (Crest-Ensae) $(31)(+1)$ & 5 & 54.83 & 42.8 \\
\hline Non-Grecsta (Crest-Ensae) $(36)(+6)$ & 6 & $49 \cdot 50$ & 38.6 \\
\hline Eco. Pub. (Inra Vers-Grig) $(12)(+2)$ & 7 & 43.11 & 33.6 \\
\hline Greqam (Aix Marseille 2-3) (45)(-4) & 8 & 42.34 & 33.0 \\
\hline Greghec $(\mathrm{Hec})(75)(+6)$ & 9 & & 29.9 \\
\hline (Ec. Polytechnique) $(34)(-3)$ & 10 & & 28.5 \\
\hline Centre (Ens Cachan) (7) $(-3)$ & 11 & 34.33 & 26.8 \\
\hline Centre (Cired) (14)(+6) & 12 & & 26.1 \\
\hline$(138)(-2)$ & 13 & 29.11 & 22.7 \\
\hline$($ Inra Rennes) $(12)(-1)$ & 14 & 28.59 & 22.3 \\
\hline Lameta (Montpellier 1-Inra) $(33)(+16)$ & 15 & 22.34 & $17 \cdot 4$ \\
\hline Thema (Cergy Pontoise) $(36)(-6)$ & 16 & 22.19 & $17 \cdot 3$ \\
\hline & 17 & 22.02 & 17.2 \\
\hline$(+12)$ & 18 & 20.63 & 16.1 \\
\hline & 19 & 19.52 & 15.2 \\
\hline ira) $(15)(+8)$ & 20 & 18.86 & 14.7 \\
\hline & 21 & 18.11 & 14.1 \\
\hline & 22 & 17.96 & 14.0 \\
\hline$-E c o($ Dijon $)(12)(+59)$ & 23 & $17 \cdot 71$ & 13.8 \\
\hline Cermes (Paris 11) $(16)(+31)$ & 24 & 17.59 & 13.7 \\
\hline & 25 & 17.55 & 13.7 \\
\hline Strasb. 1) $(72)(+1)$ & 26 & 16.99 & $13 \cdot 3$ \\
\hline & 27 & 16.76 & 13.1 \\
\hline$(-6)$ & 28 & 16.38 & 12.8 \\
\hline & 29 & 15.97 & 12.5 \\
\hline 5$)(-14)$ & 30 & 13.98 & 10.9 \\
\hline$(+28)$ & 31 & 13.79 & 10.8 \\
\hline 16) & 32 & 13.47 & 10.5 \\
\hline Ce & 33 & 13.32 & 10.4 \\
\hline & 34 & 12.92 & 10.1 \\
\hline & 35 & 12.81 & 10.0 \\
\hline 1) $(20)(+54)$ & 36 & 12.62 & 9.9 \\
\hline$(+64)$ & 37 & 12.36 & 9.6 \\
\hline & 38 & 12.26 & 9.6 \\
\hline$(37)(-5)$ & 39 & 11.90 & $9 \cdot 3$ \\
\hline & 40 & 11.47 & 8.9 \\
\hline t & 41 & 11.45 & 8.9 \\
\hline & 42 & 11.43 & 8.9 \\
\hline 8$)(-29)$ & 43 & 11.20 & 8.7 \\
\hline oly. Lille) (36) & 44 & 9.93 & $7 \cdot 7$ \\
\hline$(e-3)(13)(+3$ & $\begin{array}{l}44 \\
45\end{array}$ & $\begin{array}{l}9.93 \\
9.82\end{array}$ & $7 \cdot 7$ \\
\hline & 46 & $9 \cdot 57$ & $7 \cdot 5$ \\
\hline & 47 & 9.41 & $7 \cdot 3$ \\
\hline y) $(19)(+54)$ & 48 & 9.20 & 7.2 \\
\hline & 49 & 9.00 & 7.0 \\
\hline (e) & 50 & 8.62 & 6.7 \\
\hline$(56)(-6)$ & 51 & 8.22 & 6.4 \\
\hline nra) (29)(+38) & 52 & & 6.3 \\
\hline 4) $(35)(+9)$ & & 8.08 & 6.3 \\
\hline$(-21)$ & 54 & 8.03 & 6.3 \\
\hline$(43)(+25)$ & 55 & 7.97 & 6.2 \\
\hline & & 7.85 & 6.1 \\
\hline$(7)(+7)$ & & $7 \cdot 73$ & 6.0 \\
\hline nra) $(48)(+34)$ & 58 & $7 \cdot 48$ & 5.8 \\
\hline r 3) $(10)(-23)$ & 59 & $7 \cdot 43$ & 5.8 \\
\hline נד & 60 & 7.41 & 5.8 \\
\hline es 1$)(82)(-21)$ & 61 & 7.17 & \\
\hline 7$)(-13)$ & 62 & 6.87 & $5 \cdot 4$ \\
\hline$(24)(-22)$ & 63 & 6.76 & $5 \cdot 3$ \\
\hline Centre (St Etienne) $(22)(-10)$ & & 6.61 & \\
\hline
\end{tabular}


suite de la page précédente

\begin{tabular}{|c|c|c|c|}
\hline centre & rg. & tot. & nor. \\
\hline Autre (Montpellier 1-Inra) (21)(-16) & 65 & 108.4 & 1.4 \\
\hline Non-Crem (Caen-Rennes 1) (39)(-7) & 66 & 108.3 & 1.4 \\
\hline Centre (Rouen) $(22)(+19)$ & 67 & 106.9 & 1.4 \\
\hline Centre (Limoges) (18)(-10) & 68 & 96.5 & 1.3 \\
\hline Centre (Lille 3) (11)(-14) & 69 & 88.4 & 1.2 \\
\hline Centre (La Reunion) (19)(-19) & 70 & $87 \cdot 4$ & 1.2 \\
\hline Centre (Perpignan) (12) (-23) & 71 & $85 \cdot 3$ & 1.1 \\
\hline Lef (Inra Nancy) (7) $(+15)$ & 72 & 84.0 & 1.1 \\
\hline Non-Beta (Nancy 2-Strasb. 1) (24)(+4) & 73 & 77.1 & 1.0 \\
\hline Non-Cepn (Paris 13) (12)(+4) & 74 & 75.4 & 1.0 \\
\hline Centre (Angers) $(18)(+5)$ & 75 & $73 \cdot 3$ & 1.0 \\
\hline Centre (Montpellier 3) (10)(-6) & 76 & 70.6 & 0.9 \\
\hline Centre (La Rochelle) (5)(-8) & 77 & 69.9 & 0.9 \\
\hline Moisa (Montpellier 1-Inra) $(8)(+1)$ & 78 & 69.0 & 0.9 \\
\hline Non-Gredeg (Nice) (12)(-6) & 79 & 63.4 & 0.8 \\
\hline Centre (Toulon) (11) $(-5)$ & 80 & 63.2 & 0.8 \\
\hline Centre (Poitiers) (27) (-29) & 81 & 61.3 & 0.8 \\
\hline Centre (Tours) (12)(-21) & 82 & 59.2 & 0.8 \\
\hline Autre (Dijon) $(11)(+14)$ & 83 & 58.9 & 0.8 \\
\hline Centre (Littoral) (13)(-22) & 84 & 54.2 & 0.7 \\
\hline Centre (Rennes 2) $(7)(+4)$ & 85 & 54.1 & 0.7 \\
\hline Centre (Marne La Vallee) (11)(-19) & 86 & $53 \cdot 3$ & 0.7 \\
\hline Centre (Mulhouse) (9)(-15) & 87 & 52.4 & 0.7 \\
\hline Centre (Toulouse 2) (10)(+15) & 88 & 52.2 & 0.7 \\
\hline Centre (Brest) (21) $(-25)$ & 89 & 51.2 & 0.7 \\
\hline Centre (Ant. Guy.) (22)(-25) & 90 & 50.5 & 0.7 \\
\hline Centre (Valenciennes) $(8)(+3)$ & 91 & 48.0 & 0.6 \\
\hline Centre (Le Havre) (15)(-4) & 92 & 42.5 & 0.6 \\
\hline Centre (Cnam) (7) (-11) & 93 & 42.4 & 0.6 \\
\hline Centre (Artois) (13)(-13) & 94 & 39.8 & 0.5 \\
\hline Centre (Metz) (13)(-11) & 95 & 38.6 & 0.5 \\
\hline Centre (Paris 5) (11)(-20) & 96 & 33.1 & 0.4 \\
\hline Centre (Amiens) (22)(-14) & 97 & 29.7 & 0.4 \\
\hline Centre (Toulouse 3$)(10)(+2)$ & 98 & $27 \cdot 7$ & 0.4 \\
\hline Non-Economix (Paris 10) (15)(-6) & 99 & 26.4 & 0.4 \\
\hline Centre (Lyon 1) (5)(-4) & 100 & $24 \cdot 5$ & 0.3 \\
\hline Centre (Corte) (9)(-3) & 101 & 15.9 & 0.2 \\
\hline Centre (Bretagne Sud) (9)(-11) & 102 & $13 \cdot 3$ & 0.2 \\
\hline \multirow[t]{2}{*}{ Centre (Lyon 3) $(9)(-1)$} & 103 & 8.1 & 0.1 \\
\hline & 104 & & \\
\hline
\end{tabular}

\begin{tabular}{|c|c|c|c|}
\hline centre & rg. & p.c. & nor. \\
\hline Centre (Valenciennes) $(8)(+10)$ & 65 & 6.40 & 5.0 \\
\hline Non-Cepn (Paris 13) $(12)(+2)$ & 66 & 6.28 & 4.9 \\
\hline Centre (Reims) (32) $(-5)$ & 67 & 6.27 & 4.9 \\
\hline Centre (Mulhouse) (9)(-33) & 68 & 6.16 & 4.8 \\
\hline Centre (Cnam) (7)(-22) & 69 & 6.05 & 4.7 \\
\hline Autre (Pse-Paris 1) (33) $(+18)$ & 70 & 5.81 & 4.5 \\
\hline Centre (Toulon) (11) (-14) & 71 & 5.75 & $4 \cdot 5$ \\
\hline Autre (Lille 1-Poly. Lille) (31) $(-35)$ & 72 & 5.68 & $4 \cdot 4$ \\
\hline Centre (Toulouse 2) $(10)(+30)$ & 73 & 5.49 & $4 \cdot 3$ \\
\hline Autre (Dijon) (11)(+20) & 74 & $5 \cdot 36$ & 4.2 \\
\hline Centre (Limoges) (18)(-13) & 75 & 5.36 & 4.2 \\
\hline ice) $(12)(-16)$ & 76 & 5.29 & 4.1 \\
\hline er 1 -Inra) $(21)(-26)$ & 77 & 5.16 & 4.0 \\
\hline Marne La Vallee) (11) $(-4$ & 78 & 5.08 & 4.0 \\
\hline Lem (Lille 1-Poly. Lille) $(87)(-1)$ & 79 & 5.02 & 3.9 \\
\hline (Tours) $(12)(-41)$ & 80 & 4.93 & 3.9 \\
\hline 1) $(5)(-16)$ & 81 & 4.91 & 3.8 \\
\hline 22$)(+13)$ & 82 & 4.86 & 3.8 \\
\hline 12) $(30)(+3)$ & 83 & 4.73 & 3.7 \\
\hline ion) $(19)(-34)$ & 84 & 4.60 & 3.6 \\
\hline 3) $(-41)$ & 85 & 4.17 & $3 \cdot 3$ \\
\hline ers) $(18)(-5)$ & 86 & 4.07 & 3.2 \\
\hline Marseille 2-3) (57)(+11) & 87 & 3.58 & 2.8 \\
\hline 2-Strasb. 1) (24)(-1) & 88 & 3.28 & 2.6 \\
\hline 2-Inra) $(38)(-19)$ & 89 & 3.21 & 2.5 \\
\hline 3) $(-18)$ & 90 & 3.19 & 2.5 \\
\hline (11) $(-35)$ & 91 & 3.16 & 2.5 \\
\hline 13$)(-16)$ & 92 & 3.09 & 2.4 \\
\hline$(15)(-9)$ & 93 & 2.83 & 2.2 \\
\hline -Rennes 1) (39)(-6) & 94 & 2.78 & 2.2 \\
\hline$(10)(+4)$ & $\begin{array}{l}94 \\
95\end{array}$ & 2.77 & 2.2 \\
\hline$(21)(-27)$ & 96 & 2.50 & 2.0 \\
\hline Guy.) (22)(-24) & 97 & 2.35 & 1.8 \\
\hline 27)(-27) & 98 & 2.27 & 1.8 \\
\hline$($ Paris 10) $(15)(-3)$ & 99 & 1.82 & 1.4 \\
\hline & 100 & 1.77 & 1.4 \\
\hline Sud) $(9)(-24)$ & 101 & 1.48 & 1.2 \\
\hline s) $(22)(-9)$ & 102 & 1.35 & 1.1 \\
\hline Centre (Lyon 3) (9)(-3) & 103 & 0.90 & 0.7 \\
\hline Non-Cerdi (Clermont 1) (6)(o) & 104 & 0.01 & \\
\hline
\end{tabular}

Notes : La colonne "rg." donne le rang, la colonne "tot." donne le score total, "nor." le score normalisé par rapport à celui du premier classé, "p.c." donne le score par chercheur. Entre les premières parenthèses se trouve le nombre de chercheurs, la variation de classement par rapport à Clm est donnée entre les deuxièmes parenthèses. Si cette variation est supérieure ou égale à 3, le nom est typographié en gras italique, si elle est inférieure ou égale à -3, le nom est typographié en italique, sinon le nom est simplement typographié en gras.

\subsubsection{Indice G}

TABle 23 - Centres, Indice G, T=Dégressif

\begin{tabular}{lrrr}
\hline \hline centre & rg. & tot. & nor. \\
\hline Pjse (Pse-Paris 1) (43)(+2) & 1 & 96.2 & 100.0 \\
Gremaq (Tse-Toulouse 1) (59)(-1) & 2 & 92.5 & 96.2 \\
Ces (Pse-Paris 1) (138)(-1) & 3 & 76.5 & 79.6 \\
Greghec (Hec) (75)(+1) & 4 & 72.7 & 75.6 \\
Grecsta (Crest-Ensae) (31)(+1) & 5 & 68.7 & 71.5 \\
Non-Grecsta (Crest-Ensae)(36)(+6) & 6 & 63.1 & 65.7 \\
Greqam (Aix Marseille 2-3)(45)(-3) & 7 & 61.1 & 63.5 \\
Beta (Nancy 2-Strasb. 1)(72)(+2) & 8 & 51.5 & 53.5 \\
Preg (Ec. Polytechnique) (34)(-1) & 9 & 45.5 & 47.3 \\
Economix (Paris 10) (66)(-1) & 10 & 44.8 & 46.6 \\
Lerna (Tse-Toulouse 1)(19)(-4) & 11 & 43.7 & 45.5 \\
Centre (Iep Paris) (9)(+6) & 12 & 43.6 & 45.3 \\
\hline
\end{tabular}

\begin{tabular}{lccr}
\hline \hline centre & rg. & p.c. & nor. \\
\hline Centre (Iep Paris) (9)(+4) & 1 & 9.23 & 100.0 \\
Pjse (Pse-Paris 1) (43)(o) & 2 & 9.21 & 99.8 \\
Lerna (Tse-Toulouse 1) (19)(o) & 3 & 7.10 & 76.9 \\
Gremaq (Tse-Toulouse 1) (59)(-3) & 4 & 6.59 & 71.3 \\
Centre (Cired) (14)(+13) & 5 & 5.53 & 59.9 \\
Eco. Pub. (Inra Vers-Grig) (12)(+3) & 6 & 5.34 & 57.9 \\
Greqam (Aix Marseille 2-3) (45)(-3) & 7 & 5.17 & 56.0 \\
Smart (Inra Rennes) (12)(+5) & 8 & 4.95 & 53.6 \\
Preg (Ec. Polytechnique) (34)(-2) & 9 & 4.92 & 53.3 \\
Grecsta (Crest-Ensae) (31)(-4) & 10 & 4.78 & 51.8 \\
Centre (Ens Cachan) (7)(-3) & 11 & 4.35 & 47.1 \\
Iredu-Eco (Dijon) (12)(+70) & 12 & 4.02 & 43.6 \\
\hline & suite page suivante \\
\hline
\end{tabular}


suite de la page précédente

\begin{tabular}{|c|c|c|c|}
\hline entre & rg. & tot. & nor. \\
\hline Drm (Paris 9) (69) $(+14)$ & 13 & & \\
\hline Lameta (Montpellier 1-Inra) $(33)(+2)$ & 14 & & \\
\hline Gate (Lyon 2) (27)(o) & 15 & $35 \cdot 4$ & 36.8 \\
\hline Gredeg (Nice) $(71)(+1)$ & 16 & $34 \cdot 3$ & $35 \cdot 7$ \\
\hline Eco. Pub. (Inra Vers-Grig) (12)(+9) & 17 & & \\
\hline Thema (Cergy Pontoise) (36)(-7) & 18 & 32.1 & 33.4 \\
\hline Ermes (Paris 2) $(17)(+14)$ & 19 & 31.1 & 32.4 \\
\hline Centre (Le Mans) $(18)(-1)$ & 20 & 31.0 & 32.2 \\
\hline Centre (Cired) (14) $(+16)$ & 21 & 30.7 & 32.0 \\
\hline Cerdi (Clermont i) $(26)(+3)$ & 22 & 30.0 & 31.2 \\
\hline Lem (Lille 1-Poly. Lille) (87)(o) & 23 & $29 \cdot 3$ & 30.5 \\
\hline ennes 1) $(82)(-11)$ & 24 & 29.1 & 30.3 \\
\hline Gael (Grenoble 2-Inra) $(15)(+17)$ & 25 & $27 \cdot 4$ & 28.5 \\
\hline Centre (Strasbourg 3) (13)(+9) & 26 & 27.2 & 28.3 \\
\hline ulous & 27 & 27.2 & 28.3 \\
\hline Leg & 28 & 26.5 & $27 \cdot 5$ \\
\hline ines) $(12)(+1)$ & 29 & 26.2 & $27 \cdot 3$ \\
\hline & 30 & 25.8 & 26.8 \\
\hline & 31 & 24.5 & $25 \cdot 5$ \\
\hline aris 11) $(16)(+31)$ & 32 & 24.4 & 25.4 \\
\hline tQuentin) $(24)(+20)$ & 33 & 24.0 & 25.0 \\
\hline Nor & 34 & 23.9 & 24.8 \\
\hline $\mathrm{Cen}$ & 35 & 23.7 & 24.7 \\
\hline & 36 & 23.5 & $24 \cdot 5$ \\
\hline (1) & 37 & 23.3 & 24.2 \\
\hline Gre & 38 & 23.2 & 24.1 \\
\hline$(+27)$ & 39 & 22.8 & 23.7 \\
\hline$(+28)$ & 40 & 22.7 & 23.6 \\
\hline Cen & 41 & 22.2 & 23.1 \\
\hline 2) $(43)(+5)$ & 42 & 22.2 & 23.1 \\
\hline deaux 4) $(35)(-3)$ & 43 & 21.6 & 22.5 \\
\hline io) & 44 & 21.5 & 22.4 \\
\hline 3) $(5$ & 45 & 21.0 & 21.8 \\
\hline & 46 & 20.9 & 21.7 \\
\hline Poly. Lille) $(36)(-12$ & 47 & 20.9 & 21.7 \\
\hline & 48 & 20.3 & 21.1 \\
\hline$(19)(-20)$ & 49 & 19.1 & 19.9 \\
\hline 8) $(-14)$ & 50 & 18.7 & 19.4 \\
\hline$(+48)$ & 51 & 18.5 & 19.2 \\
\hline & 52 & 18.4 & 19.1 \\
\hline$(-11)$ & 53 & 18.4 & 19. \\
\hline & 54 & 18.1 & 18.8 \\
\hline Lille) $(31)(-27)$ & 55 & $17 \cdot 5$ & 18.2 \\
\hline & 56 & 17.2 & 17.9 \\
\hline & 57 & 17.1 & 17.8 \\
\hline & 58 & 16.8 & 17 \\
\hline es 1) (39)(o) & 59 & 16.7 & $17 \cdot 3$ \\
\hline 29$)(+14)$ & 60 & 16.4 & 17.0 \\
\hline & 61 & 16.3 & 17.0 \\
\hline$(-12)$ & 62 & 15.9 & 16.5 \\
\hline & 63 & 15.3 & 15.9 \\
\hline 18)(+16) & 64 & 15.2 & 15.8 \\
\hline & 65 & 14.6 & 15.2 \\
\hline 1 -Inra) $(21)(-17)$ & 66 & 14.4 & 14.9 \\
\hline & 67 & 14.4 & 15.0 \\
\hline & 68 & 13.8 & 14 \\
\hline & 69 & 13.5 & 14. \\
\hline$(+16)$ & 70 & 13.2 & 13.8 \\
\hline 1) $(19)(-20)$ & 71 & 13.1 & 13.6 \\
\hline$(8)(+22)$ & 72 & 13.0 & 13.6 \\
\hline a) $(38)(-30)$ & 73 & 12.9 & 13.4 \\
\hline & 74 & 12.9 & 13. \\
\hline er 1-Inra) $(8)(+4)$ & 75 & 12.8 & 13. \\
\hline 2-Strasb. 1) (24) & 76 & 12.8 & $13 \cdot 3$ \\
\hline 2) $(12)(-29)$ & 77 & 12.5 & 13.0 \\
\hline & 78 & 12.2 & 12.6 \\
\hline & 79 & & 12.4 \\
\hline 7) $(-28)$ & 80 & 11.8 & 12.3 \\
\hline Centre (To & 81 & 11.7 & 12.2 \\
\hline
\end{tabular}

\begin{tabular}{|c|c|c|c|}
\hline ntre & rg. & p.c. & nor. \\
\hline Grecsta (Crest-Ensae) $(36)(-1)$ & 13 & 3.90 & 42 \\
\hline Ces (Pse-Paris 1) (138)(-3) & 14 & 3.87 & \\
\hline Cerdi (Clermont 1) $(26)(+15)$ & 15 & 3.64 & $39 \cdot 4$ \\
\hline Greghec $(\mathrm{Hec})(75)(-1)$ & 16 & $3 \cdot 51$ & 38.0 \\
\hline Thema (Cergy Pontoise) (36)(-7) & 17 & 3.47 & 37.6 \\
\hline Gate (Lyon 2) (27)(+5) & 18 & 3.46 & $37 \cdot 4$ \\
\hline Lef (Inra Nancy) $(7)(+34)$ & 19 & 3.46 & $37 \cdot 5$ \\
\hline Centre (Strasbourg 3) (13)(-3) & 20 & 3.24 & 35.1 \\
\hline Economix (Paris 10) (66)(o) & 21 & 3.24 & 35.1 \\
\hline Centre (Chambery) $(15)(+37)$ & 22 & 3.22 & 34.9 \\
\hline Cepn (Paris 13) $(33)(+25)$ & 23 & 3.00 & 32.5 \\
\hline Cermes (Paris 11) $(21)(+77)$ & 24 & 2.90 & 31.4 \\
\hline $2-\operatorname{In} r a)(15)(+3)$ & 25 & 2.85 & 30.9 \\
\hline Beta (Nancy 2-Strasb. 1) (72)(+1) & 26 & 2.84 & 30.8 \\
\hline Lest-Eco (Aix Marseille 2-3) (13)(+55) & 27 & 2.75 & 29.8 \\
\hline s a (Montpellier 1-Inra) (8) $(+$ & 28 & 2.68 & 29.0 \\
\hline lier 1-Inra) $(33)(+2)$ & 29 & 2.56 & $27 \cdot 7$ \\
\hline 4) $(37)(+4)$ & 30 & 2.52 & $27 \cdot 3$ \\
\hline ulouse 1) $(20)(+59)$ & 31 & 2.51 & 27.2 \\
\hline$(5)(-16)$ & 32 & 2.48 & 26.9 \\
\hline$(12)(-4)$ & 33 & 2.43 & 26.3 \\
\hline$(24)(-8)$ & 34 & 2.39 & 25.8 \\
\hline ntin) $(24)(+32)$ & 35 & 2.36 & 25.6 \\
\hline & 36 & 2.33 & $25 \cdot 3$ \\
\hline & 37 & 2.33 & $25 \cdot 3$ \\
\hline 3$)(10)(-2)$ & 38 & 2.32 & 25.2 \\
\hline 11) $(16)(+17)$ & 39 & 2.32 & 25.1 \\
\hline & 40 & 2.30 & 24.9 \\
\hline$(7)(+23)$ & 41 & 2.27 & 24.6 \\
\hline 8) $(-10)$ & 42 & 2.21 & 23.9 \\
\hline$(11)(-24)$ & 43 & 2.20 & 23.8 \\
\hline $2-\operatorname{Inra})(29)(+46)$ & 44 & 2.18 & 23.6 \\
\hline & 45 & 2.17 & 23.5 \\
\hline 1) $(82)(-6)$ & 46 & 2.16 & 23.4 \\
\hline 27) & 47 & 2.15 & $23 \cdot 3$ \\
\hline 1) $(23)(-26)$ & 48 & 2.12 & 23.0 \\
\hline-15$)$ & 49 & 2.12 & 23.0 \\
\hline 9) $(-26)$ & 50 & 2.08 & 22.6 \\
\hline (Lille 1-Poly. Lille) $(36)(+7)$ & 51 & 2.02 & 21.8 \\
\hline$($ Paris 13$)(12)(+16)$ & 52 & 1.99 & 21.6 \\
\hline Ivry) $(19)(+49)$ & 53 & 1.93 & 20.9 \\
\hline 7) $(-7)$ & 54 & 1.92 & 20.8 \\
\hline -25) & 55 & 1.92 & 20.8 \\
\hline+10$)$ & 56 & 1.86 & 20.2 \\
\hline$(-14)$ & 57 & 1.85 & 20.0 \\
\hline 9) $(56)(-13)$ & 58 & 1.84 & 19.9 \\
\hline $2-$ Inra) $(48)(+33$ & 59 & 1.82 & 19.8 \\
\hline & 60 & 1.74 & 18.8 \\
\hline$(22)(-7)$ & 61 & 1.73 & 18.7 \\
\hline & 62 & 1.68 & 18.2 \\
\hline & 63 & 1.67 & 18.1 \\
\hline$(43)(+16)$ & 64 & 1.66 & 18.0 \\
\hline 7) $(-16)$ & 65 & 1.62 & $17 \cdot 5$ \\
\hline 1) $(-8)$ & 66 & 1.62 & $17 \cdot 5$ \\
\hline e) $(12)(-7)$ & 67 & 1.60 & $17 \cdot 3$ \\
\hline a Vallee) $(11)(-30)$ & 68 & 1.56 & 16.9 \\
\hline & 69 & 1.51 & 16.4 \\
\hline & 70 & 1.49 & 16.2 \\
\hline o) $(+33)$ & 71 & 1.49 & 16.2 \\
\hline$(33)(+16)$ & 72 & 1.45 & $15 \cdot 7$ \\
\hline$(+22)$ & 73 & 1.42 & 15.4 \\
\hline$(24)(-33)$ & 74 & 1.39 & 15.0 \\
\hline er 1-Inra) $(21)(-24)$ & 75 & 1.38 & 14.9 \\
\hline & 76 & 1.36 & 14.8 \\
\hline ille) $(87)(+2)$ & & 1.36 & 14.7 \\
\hline Lille) $(31)(-$ & 78 & 1.35 & 14.6 \\
\hline$(13)(-35)$ & 79 & 1.34 & 14.5 \\
\hline & 80 & 1.29 & \\
\hline$(15)(+2)$ & 81 & 1.28 & 13.8 \\
\hline
\end{tabular}


suite de la page précédente

\begin{tabular}{lrrr}
\hline \hline centre & rg. & tot. & nor. \\
\hline Centre (Artois) (13)(-1) & 82 & 11.1 & 11.5 \\
Centre (Brest) (21) $(-18)$ & 83 & 11.1 & 11.5 \\
Autre (Dijon) (11)(+13) & 84 & 10.6 & 11.0 \\
Centre (Marne La Vallee) (11)(-18) & 85 & 10.4 & 10.8 \\
Centre (Toulouse 2) (10)(+18) & 86 & 10.4 & 10.8 \\
Non-Gredeg (Nice) (12)(-14) & 87 & 10.3 & 10.7 \\
Centre (Le Havre) (15)(0) & 88 & 10.1 & 10.5 \\
Non-Cepn (Paris 13)(12)(-11) & 89 & 9.9 & 10.3 \\
Centre (Metz) (13)(-6) & 90 & 9.7 & 10.1 \\
Centre (Ant. Guy.)(22)(-26) & 91 & 9.6 & 10.0 \\
Centre (Cnam) (7)(-10) & 92 & 9.4 & 9.8 \\
Centre (La Rochelle)(5)(-24) & 93 & 9.3 & 9.7 \\
Centre (Rennes 2) (7)(-4) & 94 & 9.3 & 9.7 \\
Centre (Paris 5) (11)(-19) & 95 & 8.1 & 8.4 \\
Non-Economix (Paris 10)(15)(-3) & 96 & 7.8 & 8.1 \\
Centre (Littoral) (13)(-35) & 97 & 7.6 & 7.9 \\
Centre (Toulouse 3)(10)(+2) & 98 & 5.9 & 6.1 \\
Centre (Corte) (9)(-1) & 99 & 5.2 & 5.4 \\
Centre (Lyon 3) (9)(+3) & 100 & 5.2 & 5.4 \\
Centre (Lyon 1) (5)(-5) & 101 & 4.6 & 4.8 \\
Centre (Amiens) (22)(-19) & 102 & 4.5 & 4.7 \\
Centre (Bretagne Sud) (9)(-12) & 103 & 4.2 & 4.4 \\
& 104 & & \\
\hline
\end{tabular}

\begin{tabular}{lrrr}
\hline \hline centre & rg. & p.c. & nor. \\
\hline Centre (Tours) (12)(-43) & 82 & 1.21 & 13.1 \\
Autre (Grenoble 2-Inra) (38)(-13) & 83 & 1.20 & 13.0 \\
Centre (Limoges) (18)(-23) & 84 & 1.19 & 12.8 \\
Autre (Dijon) (11)(+9) & 85 & 1.01 & 11.0 \\
Centre (Toulouse 3)(10)(+14) & 86 & 1.01 & 10.9 \\
Centre (Valenciennes) (8)(-10) & 87 & 1.01 & 11.0 \\
Autre (Aix Marseille 2-3) (57)(+10) & 88 & 1.00 & 10.8 \\
Centre (Paris 5) (11)(-33) & 89 & 0.93 & 10.1 \\
Centre (Lyon 1) (5)(-25) & 90 & 0.92 & 9.9 \\
Non-Beta(Nancy 2-Strasb. 1) (24)(-4) & 91 & 0.91 & 9.9 \\
Centre (Metz)(13)(-16) & 92 & 0.88 & 9.5 \\
Centre (Poitiers)(27)(-21) & 93 & 0.88 & 9.5 \\
Centre (Brest) (21)(-25) & 94 & 0.83 & 9.0 \\
Centre (Corte) (9)(+2) & 95 & 0.83 & 9.0 \\
Centre (Ant. Guy.)(22)(-23) & 96 & 0.81 & 8.8 \\
Non-Crem (Caen-Rennes 1) (39)(-8) & 97 & 0.81 & 8.7 \\
Centre (Angers) (18)(-17) & 98 & 0.78 & 8.5 \\
Centre (Bretagne Sud)(9)(-22) & 99 & 0.77 & 8.3 \\
Centre (Artois) (13)(-28) & 100 & 0.75 & 8.1 \\
Centre (Amiens)(22)(-8) & 101 & 0.63 & 6.8 \\
Non-Economix (Paris 10) (15)(-6) & 102 & 0.52 & 5.6 \\
Centre (Lyon 3) (9)(-3) & 103 & 0.45 & 4.9 \\
Non-Cerdi (Clermont 1) (6)(o) & 104 & 0.01 & 0.1 \\
\hline
\end{tabular}

Notes : La colonne "rg." donne le rang, la colonne "tot." donne le score total, "nor." le score normalisé par rapport à celui du premier classé, "p.c." donne le score par chercheur. Entre les premières parenthèses se trouve le nombre de chercheurs, la variation de classement par rapport à Clm est donnée entre les deuxièmes parenthèses. Si cette variation est supérieure ou égale à 3, le nom est typographié en gras italique, si elle est inférieure ou égale à -3, le nom est typographié en italique, sinon le nom est simplement typographié en gras.

\section{Variantes}

\subsection{Classements par année de carrière et sur les moins de 50 ans}

Les universités et centres de recherche français présentent des structures par âge relativement différentes. On peut se demander si celles-ci peuvent avoir une influence sur les classements obtenus et ce qu'il adviendrait de ceux-ci si l'âge des chercheurs étaient pris en compte. A cette fin, nous présentons tout d'abord en annexe des classements établis pour notre période de temps $\mathrm{T}=$ Carrière, qui ramène le score de chaque chercheur à son âge (moins 26 ans). De plus, une fraction importante de la population a un âge compris entre 55 et 65 ans. Parmi les chercheurs de cette tranche d'âge, il se trouve un certain nombre de personnes avec des stocks d'articles assez important et qui contribuent de manière substantielle au capital recherche de leur centre/université. Certains étant proches de la retraite, il est intéressant de voir où se situent les capitaux recherche des centres en l'absence des chercheurs les plus âgés, ce qui pourrait être la situation du centre ou de l'université dans quelques années. Si le rang d'un centre diminue par rapport au classement avec tous les membres cela peut indiquer, par exemple, que des recrutements sont à envisager si le centre veut maintenir sa position. Une coupure, relativement arbitraire, à 50 ans est effectuée, seuls les chercheurs dont l'âge est strictement inférieur à 50 ans étant retenus pour le calcul des scores des institutions également présentés en annexe. 


\subsection{Classements ne gardant que les 10 ou 30 chercheurs les plus productifs}

La comparaison des centres ou universités est parfois rendue difficile par les différences de taille des institutions, raison pour laquelle nous présentons à la fois des classements en volume et par chercheur donnant deux éclairages complémentaires. Pour contrôler l'effet taille (le volume de citations augmente avec le nombre de chercheurs mais les grandes institutions ont en général aussi une plus grande part de chercheurs peu productifs ce qui diminue leur productivité moyenne), nous présentons dans cette section une approche différente qui consiste à uniformiser le nombre de chercheurs de toutes les institutions en ne retenant que les 10 puis 30 chercheurs les plus productifs de chacune. Les grandes institutions gardent un certain avantage lié à leur taille, puisque la distribution de productivité de leur chercheur est alors probablement tronquée à un niveau plus élevé mais l'effet n'est cependant plus totalement mécanique. Production totale et par chercheur conduisent d'ailleurs à la même hiérarchie des institutions, hormis du fait des quelques institutions n'ayant pas au moins 10 (respectivement 30) chercheurs. Les tableaux donnant ces classements sont également donné en annexe. Là encore, si les rangs de certaines institutions varient légèrement, la hiérarchie globale est relativement maintenue, en tous les cas dans le haut du classement. Les variations deviennent plus importantes lorsqu'on les descend, ce qui peut donner des indications utiles quant à la distribution de la production à l'intérieur des institutions.

\section{Conclusions}

Le résumé des résultats que nous obtenons est donné à la fin de l'introduction de cet article. La conclusion principale est que l'approche GS ne modifie pas fondamentalement l'image obtenue de la recherche française en économie et la hiérarchie en termes de publications académiques des centres de recherche et universités français. Pour certaines institutions dont l'activité n'est pas au cœur de l'économie, les évolutions sont un peu plus sensibles, ce qui peut faire de GS un instrument complémentaire intéressant d'Econlit, en espérant que ses limites techniques et erreurs de mesure soient rapidement comblées.

Une fois l'outil de mesure défini et ses propriétés étudiées et comprises, une étape importante, mais cependant complexe, reste à effectuer. Elle consiste en l'étude des déterminants de l'output en termes de publications des différents centres de recherche ou universités français. Il s'agit de la tâche que nous essayons de mener dans Bosquet et Combes (2011b). Nous expliquons successivement le fait qu'un chercheur publie, la quantité qu'il publie et la qualité de ses publications à la fois par un certain nombre de variables individuelles, trouvées classiquement en économie du travail comme le sexe et l'âge, et par des effets locaux liés aux caractéristiques des institutions auxquelles il est affilié. Les effets qui sont plus particulièrement étudiés sont ceux de la taille totale (nombre de chercheurs) de l'institution et de ses spécialisation et diversité thématiques, qui peuvent être 
appréhendés facilement et précisément en économie via les codes JEL présents dans la base de données Econlit. Nous contrôlons pour l'importance de l'activité d'enseignement et de recherche via le nombre d'étudiants formés par chercheur. Le rôle de l'hétérogénéité des centres en termes de productivité de leurs chercheurs, et la présence ou non de "stars", est un autre exemple d'élément pouvant affecter la productivité locale des chercheurs que nous étudions. Une fois le diagnostic de diversité des profils de publication posé, passer à l'étape de quantification des déterminants de cette diversité est certainement primordial en termes de politiques d'éducation et de recherche.

\section{Références}

Clément Bosquet et Pierre-Philippe Combes : Comparaison des mesures Econlit et Google Scholar de la production de recherche en économie en France en 2008. Direction Générale de la Recherche et de l'Innovation (DGRI) du Ministère de l'Enseignement Supérieur et de la Recherche, 2011 a.

Clément Bosquet et Pierre-Philippe Combes : Do large departments make academics more productive? Agglomeration and peer effects in research. Mimeo Greqam, $2011 b$.

Clément Bosquet, Pierre-Philippe Combes et Laurent Linnemer : La publication d'articles de recherche en économie en France en 2008. Disparités actuelles et évolutions depuis 1998. Direction Générale de la Recherche et de l'Innovation (DGRI) du Ministère de l'Enseignement Supérieur et de la Recherche, 2010.

Pierre-Philippe Combes et Laurent LinNemer : La publication d'articles de recherche en économie en France. Annales d'Économie et de Statistiques, 62:5-47, Avril/Juin 2001.

Pierre-Philippe Combes et Laurent Linnemer : L'impact international des articles de recherche français en économie. Revue Économique, 54:181-217, 2003 .

Pierre-Philippe Combes et Laurent Linnemer : Where are the economists who publish ? Publication concentration and rankings in Europe based on cumulative publications. Journal of the European Economic Association, 1(6):1250-1308, December 2003b.

Pierre-Philippe Combes et Laurent Linnemer : Inferring missing citations. a quantitative multicriteria ranking of all journals in economics. Document de travail Greqam, (2010-25), 2010.

Leo EGGHE : Theory and practise of the g-index. Scientometrics, 69:131-152, 2006.

Anne-Wil Harzing : Publish or Perish. http ://www.harzing.com/pop.htm, 2007.

Jorge Hirsh : An index to quantify an individual's scientific research output. Proceedings of the National Academy of Sciences, 102:16569-16572, 2005.

A.J. LotKA : The frequency distribution of scientific productivity. Journal of the Whashington Academy of Sciences, 16(12):317-23, 1926.

Michel Lubrano, Luc Bauwens, Alan Kirman et Camelia Protopopescu : Ranking European economic departments : a statistical approach. Journal of the European Economic Association, 1(6):13671401, December 2003.

Michael SCHREIBER : To share the fame in a fair way, $\mathrm{hm}$ modifies $\mathrm{h}$ for multi-authored manuscripts. New Journal of Physics, 10:040201-1-8, 2008. 


\title{
Annexes
}

\section{A Classements par année de carrière et sur les moins de 50 ans}

\author{
A.1 Centres, $\mathrm{T}=$ Carrière
}

TABLE 24 - Centres, Citations totales, $\mathrm{T}=$ Carrière

\begin{tabular}{|c|c|c|c|}
\hline centre & rg. & tot. & nor. \\
\hline Gremaq (Tse-Toulouse 1) (59)(o) & 1 & 2101.1 & 100.0 \\
\hline Pjse (Pse-Paris 1) (43)(o) & 2 & $1347 \cdot 7$ & 64.1 \\
\hline Ces (Pse-Paris 1) (138)(o) & 3 & 898.1 & 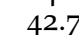 \\
\hline Greghec $(\mathrm{Hec})(75)(+2)$ & 4 & 756.3 & 36.0 \\
\hline Grecsta (Crest-Ensae) (31)(o) & 5 & 539.8 & $25 \cdot 7$ \\
\hline Greqam (Aix Marseille 2-3) (45)(-2) & 6 & $449 \cdot 4$ & 21.4 \\
\hline Preg (Ec. Polytechnique) $(34)(+1)$ & 7 & 346.4 & 16.5 \\
\hline Lerna (Tse-Toulouse 1) (19)(+1) & 8 & 321.0 & $15 \cdot 3$ \\
\hline Economix (Paris 10) $(66)(-2)$ & 9 & $319 \cdot 3$ & 15.2 \\
\hline Centre (Iep Paris) (9) $(+5)$ & 10 & 285.7 & 13.6 \\
\hline Non-Grecsta (Crest-Ensae) $(36)(+2)$ & 11 & 282.7 & 13.5 \\
\hline Beta (Nancy 2-Strasb. 1) (72) (-2) & 12 & 278.5 & $13 \cdot 3$ \\
\hline Thema (Cergy Pontoise) $(36)(-2)$ & 13 & 224.4 & 10.7 \\
\hline Drm (Paris 9) $(69)(+20)$ & 14 & 221.5 & 10.5 \\
\hline$(82)(-3)$ & 15 & 169.2 & 8.1 \\
\hline & 16 & 61.3 & $7 \cdot 7$ \\
\hline Non-Drm (Paris 9) $(56)(-3)$ & 17 & 149.0 & 7.1 \\
\hline Eco. Pub. (Inra Vers-Grig) $(12)(+4)$ & 18 & 139.8 & 6.7 \\
\hline 08$)(+4)$ & 19 & 36.8 & 6.5 \\
\hline & 20 & 31.6 & 6.3 \\
\hline & 21 & 129.8 & 6.2 \\
\hline con) $(24)(-2)$ & 22 & 123.2 & $5 \cdot 9$ \\
\hline & 23 & 119.2 & 5.7 \\
\hline Lille) $(87)(+3)$ & 24 & 110.6 & $5 \cdot 3$ \\
\hline & 25 & 10.3 & $5 \cdot 3$ \\
\hline$(+15)$ & 26 & 103.9 & 4.9 \\
\hline 7) $(-8)$ & 27 & 100.9 & 4.8 \\
\hline -Inra) (33)(-4) & 28 & 97.0 & 4.6 \\
\hline & 29 & 94.7 & 4.5 \\
\hline n) $(24)(+22)$ & 30 & 91.9 & 4.4 \\
\hline & 31 & 88.6 & 4.2 \\
\hline 2) $(-6)$ & 32 & 87.9 & 4.2 \\
\hline y. Lille) $(36)(+15)$ & 33 & 87.8 & 4.2 \\
\hline & 34 & 81.5 & 3.9 \\
\hline$(23)(-7)$ & 35 & 72.0 & \\
\hline & 36 & 71.2 & $3 \cdot 4$ \\
\hline & 37 & 65.6 & 3.1 \\
\hline$(16)(+29)$ & 38 & 64.0 & 3.1 \\
\hline 3$)(-7)$ & 39 & 63.7 & 3.0 \\
\hline 4) $(35)(+4)$ & 40 & 62.3 & 3.0 \\
\hline-3$)(57)(+19)$ & 41 & 60.5 & 2.9 \\
\hline & 42 & 60.4 & 2.9 \\
\hline$(+58)$ & & 55.9 & \\
\hline & 44 & 55.0 & 2.6 \\
\hline a) $(15)(+1)$ & 45 & 55.0 & 2.6 \\
\hline$(-9)$ & 46 & 54.6 & 2.6 \\
\hline 1) $(20)(+42)$ & & 52.3 & 2.5 \\
\hline & 48 & 51.1 & 2.4 \\
\hline 3) $(+15)$ & 49 & $49 \cdot 3$ & 2.3 \\
\hline$(+36)$ & 50 & 48.4 & 2.3 \\
\hline$(+15)$ & 51 & 47.6 & 2.3 \\
\hline nne) $(22)(-6)$ & 52 & $47 \cdot 5$ & 2.3 \\
\hline e $2-\operatorname{In} r a)(29)(+23)$ & 53 & 47.1 & 2.2 \\
\hline & & $43 \cdot 7$ & 2.1 \\
\hline . Lille) $(31)(-26)$ & & 42.6 & 2.0 \\
\hline 2) $(24)(-20)$ & 56 & 41.8 & 2.0 \\
\hline & & 41.1 & 2.0 \\
\hline & & 40.8 & 1.9 \\
\hline
\end{tabular}

\begin{tabular}{|c|c|c|c|}
\hline centre & rg. & p.c. & nor. \\
\hline Gremaq (Tse-Toulouse 1) (59)(+1) & 1 & 35.49 & 100.0 \\
\hline Centre (Iep Paris) $(9)(+2)$ & 2 & 33.62 & $94 \cdot 7$ \\
\hline Pjse (Pse-Paris 1) $(43)(-2)$ & 3 & 31.23 & 88.0 \\
\hline Lerna (Tse-Toulouse 1) (19)(-1) & 4 & $17 \cdot 35$ & 48.9 \\
\hline Grecsta (Crest-Ensae) (31)(o) & 5 & $17 \cdot 30$ & 48.8 \\
\hline Eco. Pub. (Inra Vers-Grig) $(12)(+2)$ & 6 & 11.65 & 32.8 \\
\hline Preg (Ec. Polytechnique) (34)(o) & 7 & 10.14 & 28.6 \\
\hline Greghec $(\mathrm{Hec})(75)(+8)$ & 8 & 10.10 & 28.5 \\
\hline Gregam (Aix Marseille 2-3) (45)(-3) & 9 & 9.91 & 27.9 \\
\hline Non-Grecsta (Crest-Ensae) $(36)(+4)$ & 10 & 7.87 & 22.2 \\
\hline Centre (Ens Cachan) $(7)(-2)$ & 11 & 7.45 & 21.0 \\
\hline Smart (Inra Rennes) (12)(-2) & 12 & $7 \cdot 33$ & 20.6 \\
\hline Centre (Cired) (14)(+19) & 13 & 7.02 & 19.8 \\
\hline 38$)(-2)$ & 14 & 6.52 & 18.4 \\
\hline Thema (Cergy Pontoise) (36)(-2) & 15 & 6.32 & 17.8 \\
\hline Lef (Inra Nancy) (7)(+31) & 16 & 5.29 & 14.9 \\
\hline Centre (Besancon) $(24)(+2)$ & 17 & 5.13 & 14.5 \\
\hline$(26)(+3)$ & 18 & 5.06 & 14.3 \\
\hline$($ Strasbourg 3$)(13)(-4)$ & 19 & 4.98 & 14.0 \\
\hline o) $(66)(-2)$ & 20 & 4.87 & 13.7 \\
\hline 18) $(-10)$ & 21 & 4.86 & 13.7 \\
\hline+7$)$ & 22 & 4.74 & 13.4 \\
\hline$($ Lille 2$)($ & 23 & 4.65 & 13.1 \\
\hline$($ Paris 2$)($ & 24 & 4.19 & 11.8 \\
\hline 11$)^{\prime}(16)(+32)$ & 25 & 4.13 & 11.6 \\
\hline 12$)(+51)$ & 26 & 4.03 & 11.4 \\
\hline$($ Nancy 2-Strasb. 1) $(72)(+4)$ & 27 & 3.90 & 11.0 \\
\hline Centre (Vers. St Quentin) $(24)(+27)$ & 28 & 3.83 & 10.8 \\
\hline Grenoble 2-Inra) $(15)(+7)$ & 29 & 3.79 & 10.7 \\
\hline$)(-8)$ & 30 & 3.62 & 10.2 \\
\hline Paris 13) $(33)(+27)$ & 31 & $3 \cdot 34$ & 9.4 \\
\hline Paris 9) (69) $(+52)$ & 32 & 3.23 & 9.1 \\
\hline (Chambery) $(15)(+21)$ & 33 & 3.17 & 8.9 \\
\hline 1) $(23)(0)$ & 34 & 3.15 & 8.9 \\
\hline$(9)(-5)$ & 35 & 3.12 & 8.8 \\
\hline & 36 & 3.06 & 8.6 \\
\hline$(+48)$ & 37 & 2.95 & 8.3 \\
\hline lier 1-Inra) (33)(o) & 38 & 2.94 & 8.3 \\
\hline (Grenoble 2-Inra) $(48)(+53)$ & 39 & 2.88 & 8.1 \\
\hline 2)(+43) & 40 & 2.85 & 8.0 \\
\hline 4) $(37)(-4)$ & 41 & 2.77 & 7.8 \\
\hline$(11)(-22)$ & 42 & 2.76 & 7.8 \\
\hline$(21)(+60)$ & 43 & 2.73 & $7 \cdot 7$ \\
\hline & 44 & 2.66 & $7 \cdot 5$ \\
\hline o $($ Tse-Toulouse 1) $(20)(+51)$ & 45 & 2.61 & $7 \cdot 2$ \\
\hline -Eco (Lille 1-Poly. Lille) $(36)(+24)$ & 46 & 2.47 & 7.0 \\
\hline Fate $($ Lyon 2$)(43)(+26)$ & 47 & 2.42 & 6.8 \\
\hline es) $(8)(+24)$ & 48 & 2.40 & 6.8 \\
\hline$(5)(-23)$ & 49 & 2.32 & 6.5 \\
\hline Vice) $(71)(+19)$ & 50 & 2.29 & 6.4 \\
\hline$(17)(-1)$ & 51 & 2.25 & 6.3 \\
\hline$(30)(-27)$ & 52 & 2.22 & 6.3 \\
\hline Ivry) (19) $(-30)$ & 53 & 2.21 & 6.2 \\
\hline$($ Lyon 1) $(5)(+17)$ & 54 & 2.19 & 6.2 \\
\hline t Etienne) (22) $(-8)$ & 55 & 2.16 & 6.1 \\
\hline 3) $(11)(-11)$ & & 2.14 & 6.0 \\
\hline Crem (Caen-Rennes 1) (82)(-16) & 57 & 2.06 & 5.8 \\
\hline Lest-Eco (Aix Marseille 2-3) (13)(+32) & & 1.98 & 5.6 \\
\hline
\end{tabular}


suite de la page précédente

\begin{tabular}{|c|c|c|c|}
\hline centre & rg. & tot. & nor. \\
\hline Autre (Grenoble 2-Inra) (38)(-21) & 59 & 37.8 & \\
\hline Centre $(\mathrm{Pau})(17)(-2)$ & 60 & 37.1 & \\
\hline Mona-Tsv (Inra Ivry) (19)(+38) & 61 & 35.0 & \\
\hline Non-Crem (Caen-Rennes 1) (39)(-7) & 62 & 34.8 & \\
\hline Lef (Inra Nancy) (7)(+23) & 63 & 34.4 & \\
\hline Centre (Paris 12) $(30)(+6)$ & 64 & 33.2 & \\
\hline Autre (Montpellier 1-Inra) (21)(-9) & 65 & 31.4 & \\
\hline Cesaer (Inra Dijon) (11)(-24) & 66 & 30.4 & \\
\hline Centre (Paris 7) $(10)(+27)$ & 67 & 29.5 & \\
\hline Centre (La Reunion) (19)(-17) & 68 & 28.7 & \\
\hline Centre (Mulhouse) (9)(-9) & 69 & 26.6 & \\
\hline Centre (Poitiers) $(27)(-5)$ & 70 & 26.1 & \\
\hline Lest-Eco (Aix Marseille 2-3) (13)(+20) & 71 & 25.8 & \\
\hline Centre (Lille 3) (11)(-3) & 72 & 23.5 & \\
\hline Centre (Perpignan) (12)(-38) & 73 & 22.6 & \\
\hline Centre (Angers) (18)(-3) & 74 & 22.3 & \\
\hline Non-Cepn (Paris 13) $(12)(+5)$ & 75 & 21.8 & \\
\hline Centre (Ant. Guy.) (22)(-14) & 76 & 20.3 & \\
\hline Centre (Toulon) (11)(+2) & 77 & 19.5 & \\
\hline Centre (Littoral) (13)(-31) & 78 & 19.2 & \\
\hline Centre (Marne La Vallee) (11)(-26) & 79 & 18.8 & \\
\hline Centre (Valenciennes) $(8)(+13)$ & 80 & 18.0 & \\
\hline Autre (Dijon) $(11)(+7)$ & 81 & 17.8 & \\
\hline Centre (Metz) (13)(-25) & 82 & 17.6 & \\
\hline Centre (Tours) (12)(-15) & 83 & 16.9 & \\
\hline Non-Beta (Nancy 2-Strasb. 1) (24)(-2) & 84 & 16.0 & \\
\hline sellier 1-Inra) $(8)(-10)$ & 85 & 15.8 & \\
\hline Centre (Brest) $(21)(-23)$ & 86 & 14.9 & \\
\hline Centre (Le Havre) $(15)(-4)$ & 87 & 14.2 & \\
\hline Centre (Amiens) (22)(-7) & 88 & 12.6 & \\
\hline ges) $(18)(-15)$ & 89 & 12.4 & \\
\hline Centre (Toulouse 2) (10)(+14) & 90 & 12.4 & \\
\hline Centre (Toulouse 3$)(10)(+9)$ & 91 & 12.2 & \\
\hline (La Rochelle) (5)(-20) & 92 & 11.6 & \\
\hline es 2) $(7)(-1)$ & 93 & 11.4 & \\
\hline 1) $(5)(+4)$ & 94 & 10.9 & \\
\hline Non-Gredeg (Nice) (12)(-11) & 95 & 10.7 & \\
\hline Centre (Montpellier 3) (10)(-19) & 96 & $9 \cdot 7$ & \\
\hline Centre (Cnam) (7)(-7) & 97 & 8.9 & \\
\hline Centre (Paris 5) (11)(-26) & 98 & $7 \cdot 5$ & \\
\hline Centre (Artois) (13)(-3) & 99 & 7.2 & \\
\hline Centre (Corte) (9)(-5) & 100 & 4.8 & \\
\hline Non-Economix (Paris 10) (15)(-4) & 101 & 4.0 & \\
\hline Centre (Bretagne Sud) (9)(-18) & 102 & $3 \cdot 7$ & \\
\hline Centre (Lyon 3) (9)(-1) & 103 & 2.8 & \\
\hline Non-Cerdi (Clermont 1) (6)(o) & 104 & 0.1 & \\
\hline
\end{tabular}

\begin{tabular}{|c|c|c|c|}
\hline centre & rg. & p.c. & nor. \\
\hline Centre (Perpignan) (12)(-42) & 59 & 1.97 & \\
\hline Moisa (Montpellier 1-Inra) (8)(-15) & 60 & 1.97 & 5.6 \\
\hline Mona-Tsv (Inra Ivry) $(19)(+40)$ & 61 & 1.89 & $5 \cdot 3$ \\
\hline Centre (Rouen) $(22)(+26)$ & 62 & 1.87 & 5.3 \\
\hline Non-Cepn (Paris 13) $(12)(+3)$ & 63 & 1.82 & 5.1 \\
\hline Centre (Marne La Vallee) (11) $(-37)$ & 64 & 1.79 & 5.0 \\
\hline Non-Ermes (Paris 2) (24)(-23) & 65 & 1.78 & 5.0 \\
\hline Non-Gretha (Bordeaux 4) $(35)(+2)$ & 66 & 1.78 & 5.0 \\
\hline Centre (Toulon) (11)(-6) & 67 & 1.77 & 5.0 \\
\hline Lepii (Grenoble 2-Inra) $(29)(+26)$ & 68 & 1.65 & 4.7 \\
\hline Centre (Rennes 2) $(7)(-1)$ & 69 & 1.63 & 4.6 \\
\hline Centre (Paris 8) (27)(-19) & 70 & 1.62 & 4.6 \\
\hline Centre (Reims) $(32)(-5)$ & 71 & 1.62 & 4.6 \\
\hline Autre (Dijon) (11)(+4) & 72 & 1.61 & 4.6 \\
\hline Centre (La Reunion) (19)(-27) & 73 & 1.51 & $4 \cdot 3$ \\
\hline Autre (Montpellier 1-Inra) (21)(-18) & 74 & 1.49 & 4.2 \\
\hline Autre (Pse-Paris 1) (33)(+12) & 75 & 1.49 & 4.2 \\
\hline Centre (Littoral) $(13)(-43)$ & 76 & 1.48 & 4.2 \\
\hline Centre (Metz) (13)(-38) & 77 & 1.41 & 4.0 \\
\hline Centre (Tours) $(12)(-28)$ & 78 & 1.41 & 4.0 \\
\hline Autre (Lille 1-Poly. Lille) (31)(-36) & 79 & 1.37 & 3.9 \\
\hline se 2) $(10)(+22)$ & 80 & 1.30 & 3.7 \\
\hline Centre (Cnam) $(7)(-21)$ & 81 & 1.28 & 3.6 \\
\hline Lem (Lille 1-Poly. Lille) (87)(o) & 82 & 1.27 & 3.6 \\
\hline Centre (Angers) (18)(-8) & 83 & 1.24 & $3 \cdot 5$ \\
\hline e (Toulouse 3$)(10)(+15)$ & 84 & 1.22 & 3.4 \\
\hline Centre (Paris 12) $(30)(+4)$ & 85 & 1.13 & 3.2 \\
\hline Autre (Aix Marseille 2-3) (57)(+10) & 86 & 1.07 & 3.0 \\
\hline Centre (Montpellier 3) (10) $(-35)$ & 87 & 1.02 & 2.9 \\
\hline le 2 -Inra) $(38)(-25)$ & 88 & 1.01 & 2.8 \\
\hline (Poitiers) $(27)(-10)$ & 89 & 0.97 & 2.7 \\
\hline$e$ (Ant. Guy.) (22)(-26) & 90 & 0.94 & 2.7 \\
\hline (Le Havre) $(15)(-10)$ & 91 & 0.94 & 2.7 \\
\hline -Rennes 1) (39)(-11) & 92 & 0.89 & 2.5 \\
\hline Non-Gredeg (Nice) (12)(-19) & 93 & 0.89 & 2.5 \\
\hline Centre (Brest) $(21)(-32)$ & 94 & 0.73 & 2.1 \\
\hline Centre (Paris 5) (11)(-42) & 95 & 0.72 & 2.0 \\
\hline s) $(18)(-18)$ & 96 & 0.69 & 2.0 \\
\hline Non-Beta (Nancy 2-Strasb. 1) (24)(-4) & 97 & 0.68 & 1.9 \\
\hline Centre (Amiens) $(22)(-7)$ & 98 & 0.57 & 1.6 \\
\hline Centre (Artois) (13)(-3) & 99 & 0.57 & 1.6 \\
\hline Centre (Corte) (9)(-13) & 100 & 0.53 & 1.5 \\
\hline Centre (Bretagne Sud) (9)(-42) & 101 & 0.41 & 1.2 \\
\hline Centre (Lyon 3) (9) (-2) & 102 & 0.31 & 0.9 \\
\hline conomix (Paris 10) $(15)(-5)$ & 103 & 0.28 & 0.8 \\
\hline Non-Cerdi (Clermont 1) (6)(o) & 104 & 0.01 & $\mathrm{o}$ \\
\hline
\end{tabular}

Notes : La colonne "rg." donne le rang, la colonne "tot." donne le score total, "nor." le score normalisé par rapport à celui du premier classé, "p.c." donne le score par chercheur. Entre les premières parenthèses se trouve le nombre de chercheurs, la variation de classement par rapport à Clm est donnée entre les deuxièmes parenthèses. Si cette variation est supérieure ou égale à 3, le nom est typographié en gras italique, si elle est inférieure ou égale à -3, le nom est typographié en italique, sinon le nom est simplement typographié en gras.

\section{A.2 Universités, $\mathrm{T}=$ Carrière}

TABLE 25 - Universités, Citations totales, T=Carrière

\begin{tabular}{lcrr}
\hline \hline université & rg. & p.c. & nor. \\
\hline Iep Paris (9)(0) & 1 & 33.62 & 100.0 \\
Tse-Toulouse 1 (125)(o) & 2 & 20.51 & 61.0 \\
Crest-Ensae (67)(o) & 3 & 12.26 & 36.5 \\
Inra Vers-Grig (12)(+2) & 4 & 11.65 & 34.7 \\
Pse-Paris 1 (214)(-1) & 5 & 10.73 & 31.9 \\
Ec. Polytechnique (34)(-1) & 6 & 10.14 & 30.2 \\
Hec (75)(+5) & 7 & 10.10 & 30.0 \\
Ens Cachan $(7)(-1)$ & 8 & 7.45 & 22.2 \\
\hline & suite page suivante \\
\hline
\end{tabular}


suite de la page précédente

\begin{tabular}{|c|c|c|c|}
\hline université & rg. & tot. & nor. \\
\hline Nancy 2-Strasbourg I (95)(-1) & 9 & 294.5 & 11.5 \\
\hline Iep Paris $(9)(+8)$ & 10 & 285.7 & 11.2 \\
\hline Grenoble 2-Inra $(128)(+4)$ & 11 & 276.6 & 10.8 \\
\hline Lille 1-Polytech Lille (153)(o) & 12 & 241.0 & $9 \cdot 4$ \\
\hline Lyon $2(70)(+1)$ & 13 & 233.7 & 9.1 \\
\hline Cergy Pontoise (37)(-4) & 14 & 225.1 & 8.8 \\
\hline Caen-Rennes 1 (121)(-6) & 15 & 204.0 & 8.0 \\
\hline Dijon $(65)(+15)$ & 16 & 185.4 & $7 \cdot 3$ \\
\hline Nice $(83)(+6)$ & 17 & 171.9 & 6.7 \\
\hline Bordeaux $4(72)(-5)$ & 18 & 163.3 & 6.4 \\
\hline Montpellier 1-Inra (62)(-3) & 19 & 144.1 & 5.6 \\
\hline Inra Vers-Grig $(12)(+4)$ & 20 & 139.8 & $5 \cdot 5$ \\
\hline Paris $13(45)(+9)$ & 21 & 132.1 & 5.2 \\
\hline Clermont $1(32)(-2)$ & 22 & 131.6 & 5.2 \\
\hline Besancon $(24)(-1)$ & 23 & 123.2 & 4.8 \\
\hline Paris $11(36)(+25)$ & 24 & 120.0 & 4.7 \\
\hline Paris $2(41)(-6)$ & 25 & 113.1 & 4.4 \\
\hline Cired $(14)(+10)$ & 26 & 94.7 & 3.7 \\
\hline Versailles St Quentin (24)(+15) & 27 & 91.9 & 3.6 \\
\hline Le Mans (18)(-7) & 28 & 88.6 & 3.5 \\
\hline Inra Rennes (12)(-3) & 29 & 87.9 & $3 \cdot 4$ \\
\hline Nantes $(23)(-5)$ & 30 & 81.5 & 3.2 \\
\hline Inra Ivry $(37)(-4)$ & 31 & 75.8 & 3.0 \\
\hline Orleans $(34)(-15)$ & 32 & 68.5 & 2.7 \\
\hline Strasbourg $3(13)(-4)$ & 33 & 63.7 & 2.5 \\
\hline Lille $2(13)(+6)$ & 34 & 60.4 & 2.4 \\
\hline Evry $(18)(-7)$ & 35 & 55.0 & 2.2 \\
\hline Ens Cachan (7)(-3) & 36 & 54.6 & 2.1 \\
\hline Reims $(32)(+2)$ & 37 & 51.1 & 2.0 \\
\hline Chambery $(15)(+13)$ & 38 & 47.6 & 1.9 \\
\hline St Etienne $(22)(-2)$ & 39 & $47 \cdot 5$ & 1.9 \\
\hline Paris $8(27)(-6)$ & 40 & 43.7 & 1.7 \\
\hline Rouen $(22)(+19)$ & 41 & 41.1 & 1.6 \\
\hline Pau $(17)(+3)$ & 42 & 37.1 & 1.5 \\
\hline Inra Nancy $(7)(+22)$ & 43 & 34.4 & 1.3 \\
\hline Paris $12(30)(+10)$ & 44 & 33.2 & 1.3 \\
\hline Inra Dijon (11)(-10) & 45 & 30.4 & 1.2 \\
\hline Paris $7(10)(+23)$ & 46 & 29.5 & 1.2 \\
\hline La Reunion (19)(-6) & 47 & 28.7 & 1.1 \\
\hline Mulhouse (9)(-2) & 48 & 26.6 & 1.0 \\
\hline Poitiers $(27)(+1)$ & 49 & 26.1 & 1.0 \\
\hline Lille $3(11)(+3)$ & 50 & 23.5 & 0.9 \\
\hline Perpignan (12)(-19) & 51 & 22.6 & 0.9 \\
\hline Angers $(18)(+3)$ & 52 & 22.3 & 0.9 \\
\hline Antilles Guyane (22)(-6) & 53 & 20.3 & 0.8 \\
\hline Toulon $(11)(+7)$ & 54 & 19.5 & 0.8 \\
\hline Littoral $(13)(-17)$ & 55 & 19.2 & 0.8 \\
\hline Marne La Vallee $(11)(-13)$ & 56 & 18.8 & 0.7 \\
\hline Valenciennes $(8)(+11)$ & 57 & 18.0 & 0.7 \\
\hline Metz $(13)(-14)$ & 58 & 17.6 & 0.7 \\
\hline Tours (12)(-7) & 59 & 16.9 & 0.7 \\
\hline Brest $(21)(-12)$ & 60 & 14.9 & 0.6 \\
\hline Le Havre $(15)(+2)$ & 61 & 14.2 & 0.6 \\
\hline Amiens $(22)(\mathrm{o})$ & 62 & 12.6 & 0.5 \\
\hline Limoges $(18)(-5)$ & 63 & 12.4 & 0.5 \\
\hline Toulouse 2 (10) $(+12)$ & 64 & 12.4 & 0.5 \\
\hline Toulouse $3(10)(+8)$ & 65 & 12.2 & 0.5 \\
\hline La Rochelle $(5)(-10)$ & 66 & 11.6 & 0.5 \\
\hline Rennes 2 (7)(o) & 67 & 11.4 & 0.5 \\
\hline Lyon $1(5)(+4)$ & 68 & 10.9 & 0.4 \\
\hline Montpellier $3(10)(-10)$ & 69 & 9.7 & 0.4 \\
\hline Cnam $(7)(-4)$ & 70 & 8.9 & 0.4 \\
\hline Paris $5(11)(-15)$ & 71 & $7 \cdot 5$ & 0.3 \\
\hline Artois (13) $(-1)$ & 72 & 7.2 & 0.3 \\
\hline Corte $(9)(-3)$ & 73 & 4.8 & 0.2 \\
\hline Bretagne Sud (9)(-10) & 74 & 3.7 & 0.2 \\
\hline Lyon $3(9)(-1)$ & 75 & 2.8 & 0.1 \\
\hline
\end{tabular}

\begin{tabular}{|c|c|c|c|}
\hline université & rg. & p.c. & nor. \\
\hline Inra Rennes (12)(-1) & 9 & $7 \cdot 33$ & 21.8 \\
\hline Cired $(14)(+15)$ & 10 & 7.02 & 20.9 \\
\hline Cergy Pontoise (37)(-1) & 11 & 6.08 & 18.1 \\
\hline Inra Nancy $(7)(+25)$ & 12 & 5.29 & $15 \cdot 7$ \\
\hline Besancon $(24)(+2)$ & 13 & 5.13 & $15 \cdot 3$ \\
\hline Strasbourg $3(13)(-3)$ & 14 & 4.98 & 14.8 \\
\hline Le Mans (18)(-6) & 15 & 4.86 & 14.5 \\
\hline Aix Marseille 2-3 (115)(-2) & 16 & 4.66 & 13.9 \\
\hline Lille $2(13)(+10)$ & 17 & 4.65 & 13.8 \\
\hline Clermont $1(32)(+6)$ & 18 & 4.11 & 12.2 \\
\hline Paris 1o $(80)(-1)$ & 19 & 4.04 & 12.0 \\
\hline Versailles St Quentin $(24)(+26)$ & 20 & 3.83 & 11.4 \\
\hline Nantes $(23)(-4)$ & 21 & 3.62 & 10.8 \\
\hline Paris $11(36)(+45)$ & 22 & $3 \cdot 33$ & 9.9 \\
\hline Lyon $2(70)(+11)$ & 23 & $3 \cdot 32$ & 9.9 \\
\hline Chambery $(15)(+21)$ & 24 & 3.17 & $9 \cdot 4$ \\
\hline Mulhouse (9)(-2) & 25 & 3.12 & 9.3 \\
\hline Nancy 2-Strasbourg $1(95)(+3)$ & 26 & 3.10 & 9.2 \\
\hline Evry $(18)(-8)$ & 27 & 3.06 & 9.1 \\
\hline Paris $9(124)(+19)$ & 28 & 2.98 & 8.9 \\
\hline Paris $7(10)(+37)$ & 29 & 2.95 & 8.8 \\
\hline Paris $13(45)(+18)$ & 30 & 2.94 & 8.7 \\
\hline Dijon $(65)(+32)$ & 31 & 2.86 & 8.5 \\
\hline Paris $2(41)(-4)$ & 32 & 2.79 & 8.3 \\
\hline Inra Dijon (11)(-17) & 33 & 2.76 & 8.2 \\
\hline Valenciennes $(8)(+25)$ & 34 & 2.40 & 7.1 \\
\hline La Rochelle (5) $(-15)$ & $\begin{array}{l}34 \\
35\end{array}$ & 2.32 & 6.9 \\
\hline Montpellier 1-Inra $(62)(-4)$ & 36 & 2.32 & 6.9 \\
\hline Bordeaux $4(72)(-5)$ & 37 & 2.28 & 6.8 \\
\hline Pau $(17)(+2)$ & 38 & 2.25 & 6.7 \\
\hline Lyon $1(5)(+19)$ & 39 & 2.19 & 6.5 \\
\hline Grenoble 2-Inra (128)(+19) & 40 & 2.16 & 6.4 \\
\hline St Etienne $(22)(-3)$ & 41 & 2.16 & 6.4 \\
\hline Lille $3(11)(-9)$ & 42 & 2.14 & 6.4 \\
\hline Nice $(83)(+14)$ & 43 & 2.08 & 6.2 \\
\hline Inra Ivry $(37)(-8)$ & 44 & 2.05 & 6.1 \\
\hline Orleans $(34)(-23)$ & 45 & 2.02 & 6.0 \\
\hline Perpignan (12)(-33) & 46 & 1.97 & 5.9 \\
\hline Rouen $(22)(+22)$ & 47 & 1.87 & 5.6 \\
\hline Marne La Vallee (11)(-27) & 48 & 1.79 & $5 \cdot 3$ \\
\hline Toulon $(11)(+2)$ & 49 & 1.77 & $5 \cdot 3$ \\
\hline Caen-Rennes 1 (121)(-10) & 50 & 1.69 & 5.0 \\
\hline Rennes $2(7)(+5)$ & 51 & 1.63 & 4.8 \\
\hline Paris $8(27)(-10)$ & 52 & 1.62 & 4.8 \\
\hline Reims $(32)(+2)$ & 53 & 1.62 & 4.8 \\
\hline Lille 1-Polytech Lille $(153)(+1)$ & 54 & 1.57 & 4.7 \\
\hline La Reunion (19)(-21) & 55 & 1.51 & 4.5 \\
\hline Littoral $(13)(-30)$ & 56 & 1.48 & $4 \cdot 4$ \\
\hline Metz (13) $(-27)$ & 57 & 1.41 & 4.2 \\
\hline Tours (12)(-18) & 58 & 1.41 & 4.2 \\
\hline Toulouse $2(10)(+16)$ & 59 & 1.30 & 3.9 \\
\hline Cnam (7)(-10) & 60 & 1.28 & 3.8 \\
\hline Angers (18)(o) & 61 & 1.24 & 3.7 \\
\hline Toulouse $3(10)(+11)$ & 62 & 1.22 & 3.6 \\
\hline Paris $12(30)(+7)$ & 63 & 1.13 & $3 \cdot 4$ \\
\hline Montpellier $3(10)(-21)$ & 64 & 1.02 & 3.0 \\
\hline Poitiers $(27)(-2)$ & 65 & 0.97 & 2.9 \\
\hline Antilles Guyane $(22)(-13)$ & 66 & 0.94 & 2.8 \\
\hline Le Havre $(15)(-1)$ & 67 & 0.94 & 2.8 \\
\hline Brest $(21)(-16)$ & 68 & 0.73 & 2.2 \\
\hline Paris 5 (11)(-25) & 69 & 0.72 & 2.1 \\
\hline Limoges $(18)(-8)$ & 70 & 0.69 & 2.1 \\
\hline Amiens (22)(0) & 71 & 0.57 & 1.7 \\
\hline Artois $(13)(+1)$ & 72 & 0.57 & 1.7 \\
\hline Corte $(9)(-6)$ & 73 & 0.53 & 1.6 \\
\hline Bretagne Sud (9)(-26) & 74 & 0.41 & 1.2 \\
\hline \multirow[t]{2}{*}{ Lyon $3(9)(-1)$} & 75 & 0.31 & 0.9 \\
\hline & uite & age su & ante \\
\hline
\end{tabular}


suite de la page précédente

\begin{tabular}{l}
\hline \hline université \\
Notes : La colonne "rg." donne le rang, la colonne "tot." donne le score total, "nor." le score normalisé \\
par rapport à celui du premier classé, "p.c." donne le score par chercheur. Entre les premières paren- \\
thèses se trouve le nombre de chercheurs, la variation de classement par rapport à Clm est donnée \\
entre les deuxièmes parenthèses. Si cette variation est supérieure ou égale à 3, le nom est typographié \\
en gras italique, si elle est inférieure ou égale à -3, le nom est typographié en italique, sinon le nom \\
est simplement typographié en gras.
\end{tabular}

\section{A.3 Centres, moins de 50 ans}

TABle 26 - Centres, Moins de 50 ans, Citations totales, T=Dégressif

\begin{tabular}{|c|c|c|c|}
\hline centre & rg. & tot. & nor. \\
\hline Pjse (Pse-Paris 1$)(23)(+2)$ & 1 & 3079.8 & 100.0 \\
\hline Gremaq (Tse-Toulouse 1) $(41)(-1)$ & 2 & 2561.7 & 83.2 \\
\hline Greghec $(\mathrm{Hec})(61)(+1)$ & 3 & 2188.3 & 71.1 \\
\hline Ces (Pse-Paris 1) (80)(-2) & 4 & 2032.5 & 66.0 \\
\hline Lerna (Tse-Toulouse 1) (14) $(+1)$ & 5 & 1186.0 & 38.5 \\
\hline Greqam (Aix Marseille 2-3) (27)(-1) & 6 & 1119.0 & 36.3 \\
\hline Economix (Paris 10) $(44)(+2)$ & 7 & 839.0 & 27.2 \\
\hline Non-Grecsta (Crest-Ensae) (15) (+2) & 8 & 801.1 & 26.0 \\
\hline Beta (Nancy 2-Strasb. 1) (52)(-1) & 9 & 772.2 & 25.1 \\
\hline Thema (Cergy Pontoise) $(32)(-3)$ & 10 & 732.7 & 23.8 \\
\hline Preg (Ec. Polytechnique) (19)(o) & 11 & 664.4 & 21.6 \\
\hline Grecsta (Crest-Ensae) $(15)(+1)$ & 12 & 500.3 & 16.3 \\
\hline Crem (Caen-Rennes 1) (64)(-1) & 13 & 463.0 & 15.0 \\
\hline Eco. Pub. (Inra Vers-Grig) $(8)(+5)$ & 14 & 446.2 & $14 \cdot 5$ \\
\hline Gate (Lyon 2) (19)(+5) & 15 & 391.6 & 12.7 \\
\hline 1) $(18)(+2)$ & 16 & 361.8 & 11.8 \\
\hline Drm (Paris 9) $(43)(+8)$ & 17 & 350.9 & 11.4 \\
\hline Lem (Lille 1-Poly. Lille) (70)(-3) & 18 & 339.5 & 11.0 \\
\hline Smart (Inra Rennes) (11)(+3) & 19 & 305.2 & 9.9 \\
\hline Cen & 20 & $287 \cdot 3$ & $9 \cdot 3$ \\
\hline Clerse-Eco (Lille 1-Poly. Lille) $(23)(+10)$ & 21 & 282.4 & 9.2 \\
\hline Centre (Cired) $(7)(+20)$ & 22 & 243.6 & 7.9 \\
\hline s) $(27)(+5)$ & 23 & 242.8 & $7 \cdot 9$ \\
\hline Centre (Lille 2) $(10)(+22)$ & 24 & 231.6 & $7 \cdot 5$ \\
\hline$($ Paris 2$)(10)(+7)$ & 25 & 222.7 & 7.2 \\
\hline ) $(19)(+1)$ & 26 & 210.4 & 6.8 \\
\hline Leg & 27 & 205.2 & 6.7 \\
\hline y) $(12)(+26)$ & 28 & $204 \cdot 4$ & 6.6 \\
\hline 4) $(26)(-5)$ & 29 & 191.4 & 6.2 \\
\hline (17) $(-13)$ & 30 & 186.1 & 6.0 \\
\hline$(11)(-2)$ & 31 & 182.8 & $5 \cdot 9$ \\
\hline$(-18)$ & 32 & 181.2 & $5 \cdot 9$ \\
\hline Gredeg (Nice) $(34)(+1)$ & 33 & 179.1 & 5.8 \\
\hline Centre (Vers. St Quentin) $(17)(+16)$ & 34 & 176.6 & $5 \cdot 7$ \\
\hline c 4) $(17)(+21)$ & 35 & 171.5 & 5.6 \\
\hline a) $(35)(+9)$ & 36 & 169.0 & $5 \cdot 5$ \\
\hline n 2) $(17)(+29)$ & 37 & 168.6 & $5 \cdot 5$ \\
\hline Centre (Evry) (16)(-8) & 38 & 167.1 & $5 \cdot 4$ \\
\hline Centre (Pau) (9)(+23) & 39 & 156.1 & 5.1 \\
\hline e (Reims) (19)(o) & 40 & 150.6 & 4.9 \\
\hline Autre (Tse-Toulouse 1) (7)(-8) & 41 & 143.3 & 4.7 \\
\hline Gael (Grenoble 2-Inra) (10)(-4) & 42 & 143.0 & 4.6 \\
\hline es (Paris 11) (8)(+10) & 43 & 141.9 & 4.6 \\
\hline Toulouse 1) $(12)(+42)$ & 44 & 141.6 & 4.6 \\
\hline Aliss (Inra Ivry) (13)(-22) & 45 & 141.5 & 4.6 \\
\hline Centre (St Etienne) (17)(-10) & 46 & 141.0 & 4.6 \\
\hline renoble 2 -Inra) $(17)(+28)$ & 47 & 133.2 & $4 \cdot 3$ \\
\hline 3) $(18)(-4)$ & 48 & 128.3 & 4.2 \\
\hline Centre (Paris 8) $(13)(-12)$ & 49 & 127.4 & 4.1 \\
\hline Lameta (Montpellier 1-Inra) (17)(-34) & 50 & 126.8 & 4.1 \\
\hline Autre (Lille 1-Poly. Lille) (18)(-30) & 51 & 125.9 & 4.1 \\
\hline Centre (Rouen) $(12)(+25)$ & 52 & $97 \cdot 5$ & 3.2 \\
\hline Lest-Eco (Aix Marseille 2-3) (9)(+35) & 53 & 91.1 & 3.0 \\
\hline
\end{tabular}

\begin{tabular}{|c|c|c|c|}
\hline centre & rg. & p.c. & nor \\
\hline Pjse (Pse-Paris 1) (23)(o) & 1 & 133.61 & 100.0 \\
\hline Lerna (Tse-Toulouse 1$)(14)(+1)$ & 2 & 84.72 & 63.4 \\
\hline Gremaq (Tse-Toulouse 1) (41)(-1) & 3 & 62.03 & 46.4 \\
\hline Eco. Pub. (Inra Vers-Grig) $(8)(+2)$ & 4 & $55 \cdot 78$ & 41.8 \\
\hline Non-Grecsta (Crest-Ensae) (15)(o) & 5 & 54.87 & 41.1 \\
\hline Greqam (Aix Marseille 2-3) (27)(-2) & 6 & 42.22 & 31.6 \\
\hline Centre (Cired) $(7)(+17)$ & 7 & $37 \cdot 48$ & 28.1 \\
\hline Greghec (Hec) (61) (+5) & 8 & 35.87 & 26.0 \\
\hline Preg (Ec. Polytechnique) (19)(-2) & 9 & 34.25 & 25.6 \\
\hline Grecsta (Crest-Ensae) (15)(-1) & 10 & 32.92 & 24.6 \\
\hline Smart (Inra Rennes) (11) $(+3)$ & 11 & $27 \cdot 74$ & 20.8 \\
\hline Ces (Pse-Paris 1) (80)(-1) & 12 & 25.49 & 19.1 \\
\hline$s($ Paris & 13 & 23.44 & 17.6 \\
\hline Thema (Cergy Pontoise) (32)(-4) & 14 & 23.26 & 17.4 \\
\hline Centre (Lille 2) (10)(+25) & 15 & 23.16 & $17 \cdot 3$ \\
\hline (Tse-Toulouse 1$)(7)(-4)$ & 16 & 20.47 & 15.3 .3 \\
\hline 19) $(+13)$ & 17 & 20.19 & 15.1 \\
\hline Cerdi (Clermont 1) (18)(+8) & 18 & 20.10 & 15.0 \\
\hline Economix (Paris 10) $(44)(+3)$ & 19 & 19.27 & 14.4 \\
\hline$S 11)(8)(+15)$ & 20 & 18.92 & 14.2 \\
\hline 16) $(+10)$ & 21 & 17.96 & 13.4 \\
\hline$(\mathrm{Pau})(9)(+24)$ & 22 & $17 \cdot 34$ & 13.0 \\
\hline 12) $(+34)$ & 23 & 17.04 & 12.8 \\
\hline$(11)(-9)$ & 24 & 16.77 & 12.6 \\
\hline (Le Man & 25 & 16.10 & 12.1 \\
\hline$($ Nancy 2 -Strasb. 1) $(52)(+2)$ & 26 & 14.85 & 11.1 \\
\hline a) $(10)(+7)$ & 27 & 14.30 & 10.7 \\
\hline (La Rochelle) $(5)(-7)$ & 28 & 13.98 & 10.5 \\
\hline ellier 1-Inra) $(6)(-10)$ & 29 & 12.70 & 9.5 \\
\hline Lef (Inra Nancy) (6)(+31) & 30 & 12.44 & 9.3 \\
\hline Lirhe-Eco (Tse-Toulouse 1) (12)(+56) & 31 & 12.32 & 9.2 \\
\hline oly. Lille) $(23)(+17)$ & 32 & 12.28 & 9.2 \\
\hline$(+45)$ & 33 & 11.68 & 8.7 \\
\hline$(-16)$ & 34 & 11.32 & 8.5 \\
\hline$(-15)$ & 35 & 11.28 & 8.4 \\
\hline & 36 & 11.08 & 8.3 \\
\hline 3) $(6)(-12)$ & 37 & 10.89 & 8.2 \\
\hline nra Ivry) $(7)(+52)$ & 38 & 10.88 & 8.1 \\
\hline Evry) $(16)(-6)$ & 39 & 10.78 & 8.1 \\
\hline Vers. St Quentin) $(17)(+26)$ & 40 & 10.39 & 7.8 \\
\hline$(17)(+36)$ & 41 & 10.22 & $7 \cdot 7$ \\
\hline$(-4)$ & 42 & 10.19 & 7.6 \\
\hline a Dijon) $(8)(-14)$ & 43 & 10.12 & 7.6 \\
\hline Marseille 2-3) (9)(+45) & 44 & 10.12 & 7.6 \\
\hline (1) $(17)$ & 45 & 10.09 & 7.6 \\
\hline 1) $(6)(+43)$ & 46 & 9.79 & $7 \cdot 3$ \\
\hline se) $(6)(-20)$ & 47 & 9.42 & 7.1 \\
\hline ) $(8)(-32)$ & 48 & $9 \cdot 35$ & 7.0 \\
\hline 2) $(10)(-10)$ & 49 & 9.23 & 6.9 \\
\hline Non-Drm (Paris 9) $(27)(+4)$ & 50 & 8.99 & 6.7 \\
\hline Centre (Rennes 2) (6)(+12) & 51 & 8.94 & 6.7 \\
\hline & 52 & 8.37 & 6.3 \\
\hline Centre (St Etienne) $(17)(-2)$ & & & \\
\hline & \multicolumn{3}{|c|}{ suite page suivante } \\
\hline
\end{tabular}


suite de la page précédente

\begin{tabular}{|c|c|c|c|}
\hline centre & rg. & tot. & nor. \\
\hline Non-Ermes (Paris 2) (10)(-6) & 54 & 87.7 & 2.9 \\
\hline Cesaer (Inra Dijon) (8)(-14) & 55 & 81.0 & 2.6 \\
\hline Autre (Montpellier 1-Inra) (6)(-13) & 56 & 76.2 & 2.5 \\
\hline Centre (Paris 12) $(7)(+28)$ & 57 & $75 \cdot 9$ & 2.5 \\
\hline Centre (Perpignan) $(8)(-23)$ & 58 & 74.8 & 2.4 \\
\hline Non-Crem (Caen-Rennes 1) (25)(-12) & 59 & 72.3 & 2.4 \\
\hline Mona-Tsv (Inra Ivry) (7)(+3o) & 60 & 70.7 & 2.3 \\
\hline Centre (La Rochelle) (5)(-6) & 61 & 69.9 & 2.3 \\
\hline Lef (Inra Nancy) (6)(+20) & 62 & 68.4 & 2.2 \\
\hline Centre (Angers) (14)(+6) & 63 & 66.1 & 2.2 \\
\hline Centre (Montpellier 3) (6)(-7) & 64 & 59.9 & 2.0 \\
\hline Centre (Limoges) (9)(o) & 65 & $57 \cdot 5$ & 1.9 \\
\hline Autre (Pse-Paris 1) $(13)(+4)$ & 66 & 56.9 & 1.9 \\
\hline Centre (La Reunion) (15)(-16) & 67 & 56.8 & 1.9 \\
\hline Cermes (Paris 11) (6) $(+21)$ & 68 & $53 \cdot 9$ & 1.8 \\
\hline Centre (Rennes 2) (6) (+11) & 69 & 53.6 & 1.7 \\
\hline Centre (Littoral) (12)(-21) & 70 & 53.5 & 1.7 \\
\hline Centre (Mulhouse) $(6)(-10)$ & 71 & 51.8 & 1.7 \\
\hline he La Vallee) (10)(-14) & 72 & 51.5 & 1.7 \\
\hline Marseille 2-3) (25)(-9) & 73 & 50.3 & 1.6 \\
\hline Centre (Lille 3$)(6)(+2)$ & 74 & 46.2 & 1.5 \\
\hline Centre (Poitiers) (17) $(-11)$ & 75 & 46.2 & 1.5 \\
\hline Cent & 76 & 46.0 & 1.5 \\
\hline Ant. Guy.) $(13)(-18)$ & 77 & 41.4 & 1.3 \\
\hline e (Le Havre) $(11)(+1)$ & 78 & 40.2 & 1.3 \\
\hline Centre (Metz) (7)(-5) & 79 & 37.6 & 1.2 \\
\hline Non-Cepn (Paris 13) (7)(-9) & 80 & 36.9 & 1.2 \\
\hline ble 2-Inra) $(13)(-42)$ & 81 & 36.8 & 1.2 \\
\hline n) $(5)(-9)$ & 82 & 35.9 & 1.2 \\
\hline$(12)(-23)$ & 83 & 30.6 & 1.0 \\
\hline aris 5$)(6)(-12)$ & 84 & 28.2 & 0.9 \\
\hline Non-Beta (Nancy 2-Strasb. 1) (7)(-17) & 85 & 28.1 & 0.9 \\
\hline Centre (Lyon 1$)(5)(-2)$ & 86 & 24.5 & 0.8 \\
\hline Centre (Artois) (7)(-9) & 87 & 17.9 & 0.6 \\
\hline s) $(11)(-7)$ & 88 & 16.8 & 0.6 \\
\hline Centre (Corte) $(6)(-2)$ & 89 & 14.0 & 0.5 \\
\hline Centre (Bretagne Sud) (7)(-7) & 90 & 10.3 & 0.3 \\
\hline
\end{tabular}

\begin{tabular}{|c|c|c|c|}
\hline centre & rg. & p.c. & nor. \\
\hline Drm (Paris 9) (43)(+15) & 54 & 8.16 & 6.1 \\
\hline Centre (Rouen) $(12)(+24)$ & & 8.12 & 6.1 \\
\hline Centre (Reims) (19) $(+4)$ & 56 & 7.92 & 5.9 \\
\hline Lepii (Grenoble 2-Inra) $(17)(+28)$ & 57 & 7.83 & $5 \cdot 9$ \\
\hline Centre (Lille 3) (6)(-5) & 58 & $7 \cdot 70$ & 5.8 \\
\hline Gretha (Bordeaux 4) (26)(-11) & 59 & $7 \cdot 51$ & 5.6 \\
\hline Lameta (Montpellier 1-Inra) (17)(-37) & 60 & $7 \cdot 46$ & 5.6 \\
\hline Crem (Caen-Rennes 1) (64)(-16) & 61 & 7.23 & $5 \cdot 4$ \\
\hline Centre (Toulon) $(5)(-20)$ & 62 & 7.17 & $5 \cdot 4$ \\
\hline$($ Paris 13$)(18)(+1)$ & 63 & 7.13 & $5 \cdot 3$ \\
\hline Autre (Lille 1-Poly. Lille) (18)(-32) & 64 & 6.99 & 5.2 \\
\hline Centre (Limoges) (9)(-13) & 65 & 6.77 & 5.1 \\
\hline $\operatorname{Leg}($ Dijon $)(32)(+18)$ & 66 & 6.51 & 4.9 \\
\hline Centre (Metz) (7)(-11) & 67 & $5 \cdot 79$ & $4 \cdot 3$ \\
\hline Centre (Marne La Vallee) (10)(-21) & 68 & 5.42 & 4.1 \\
\hline Gredeg (Nice) $(34)(+2)$ & 69 & 5.27 & $3 \cdot 9$ \\
\hline epn (Paris 13) (7) $(-14)$ & 70 & 5.27 & 4.0 \\
\hline Centre (Paris 5) (6) (-28) & 71 & 5.12 & 3.8 \\
\hline Centre (Lyon 1$)(5)(-5)$ & 72 & 4.91 & 3.7 \\
\hline Cerag (Grenoble 2-Inra) (35)(+9) & 73 & 4.90 & $3 \cdot 7$ \\
\hline Poly. Lille) $(70)(+1)$ & 74 & 4.85 & 3.6 \\
\hline (Angers) $(14)(-2)$ & 75 & 4.72 & $3 \cdot 5$ \\
\hline $\operatorname{aris} 1)(13)(-4)$ & 76 & 4.55 & 3.4 \\
\hline oral) $(12)(-27)$ & 77 & 4.45 & $3 \cdot 3$ \\
\hline Jancy 2-Strasb. 1) (7)(-34) & 78 & $4 \cdot 33$ & 3.2 \\
\hline$(5)(-17)$ & 79 & 3.79 & 2.8 \\
\hline Centre (Le Havre) (11)(o) & 80 & 3.66 & 2.7 \\
\hline Centre (Ant. Guy.) (13)(-22) & 81 & $3 \cdot 31$ & 2.5 \\
\hline Autre (Grenoble 2-Inra) (13)(-41) & 82 & 2.94 & 2.2 \\
\hline Non-Crem (Caen-Rennes 1) (25)(-7) & 83 & 2.89 & 2.2 \\
\hline Centre (Poitiers) (17)(-10) & 84 & 2.72 & 2.0 \\
\hline$(12)(-27)$ & 85 & 2.66 & 2.0 \\
\hline Centre (Artois) (7)(-21) & 86 & 2.56 & 1.9 \\
\hline Centre (Corte) $(6)(-4)$ & 87 & 2.33 & 1.7 \\
\hline Autre (Aix Marseille 2-3) (25)(-2) & 88 & 2.01 & 1.5 \\
\hline ens) (11)(-8) & 89 & 1.53 & 1.1 \\
\hline Centre (Bretagne Sud) (7)(-20) & 90 & 1.48 & 1.1 \\
\hline
\end{tabular}

Notes : La colonne "rg." donne le rang, la colonne "tot." donne le score total, "nor." le score normalisé par rapport à celui du premier classé, "p.c." donne le score par chercheur. Entre les premières parenthèses se trouve le nombre de chercheurs, la variation de classement par rapport à Clm est donnée entre les deuxièmes parenthèses. Si cette variation est supérieure ou égale à 3, le nom est typographié en gras italique, si elle est inférieure ou égale à -3, le nom est typographié en italique, sinon le nom est simplement typographié en gras.

TABLE 27 - Centres, Moins de 50 ans, Indice G, T=Dégressif

\begin{tabular}{|c|c|c|c|}
\hline centre & rg. & p.c. & nor. \\
\hline Pjse (Pse-Paris 1$)(23)(0)$ & 1 & & 100.0 \\
\hline Lerna (Tse-Toulouse 1) (14)(+1) & 2 & $7 \cdot 48$ & $79 \cdot 3$ \\
\hline Centre (Cired) $(7)(+21)$ & 3 & 6.24 & 66.2 \\
\hline Eco. Pub. (Inra Vers-Grig) $(8)(+2)$ & 4 & 6.08 & $64 \cdot 4$ \\
\hline Gremaq (Tse-Toulouse 1$)(41)(-3)$ & 5 & 5.74 & 60.8 \\
\hline Non-Grecsta (Crest-Ensae) $(15)(-1)$ & 6 & $5 \cdot 30$ & 56.1 \\
\hline Greqam (Aix Marseille 2-3) (27)(-3) & 7 & 5.14 & 54.5 \\
\hline Smart (Inra Rennes) (11)(+6) & 8 & 4.61 & 48.9 \\
\hline Preg (Ec. Polytechnique) (19)(-2) & 9 & 4.38 & 46.4 \\
\hline Centre (Chambery) $(12)(+47)$ & 10 & 3.83 & 40.6 \\
\hline Grecsta (Crest-Ensae) (15)(-2) & 11 & 3.79 & 40.1 \\
\hline Ces (Pse-Paris 1) (8o) $(-1)$ & 12 & 3.66 & 38.8 \\
\hline Thema (Cergy Pontoise) $(32)(-3)$ & 13 & 3.63 & 38.5 \\
\hline Economix (Paris 10) $(44)(+8)$ & 14 & 3.55 & 37.6 \\
\hline Non-Cermes (Paris 11) $(8)(+20)$ & 15 & 3.51 & 37.2 \\
\hline Centre (Le Mans) (11)(-8) & 16 & 3.40 & 36.0 \\
\hline Greghec (Hec) (61)(-4) & 17 & $3 \cdot 31$ & 35.1 \\
\hline Cerdi (Clermont 1) (18)(+8) & 18 & 3.29 & 34.8 \\
\hline Autre (Tse-Toulouse 1) (7)(-7) & 19 & 3.26 & 34.5 \\
\hline Autre (Montpellier 1-Inra) $(6)(-1)$ & 20 & 3.23 & 34.2 \\
\hline \multicolumn{4}{|c|}{ suite page suivante } \\
\hline
\end{tabular}


suite de la page précédente

\begin{tabular}{|c|c|c|c|}
\hline e & rg. & tot. & nor. \\
\hline Cerdi (Clermont 1) (18)(-3) & 21 & 23.8 & \\
\hline Lem (Lille 1-Poly. Lille) $(70)(-7)$ & 22 & $23 \cdot 7$ & 30.6 \\
\hline Centre (Lille 2$)(10)(+23)$ & 23 & 22.9 & $29 \cdot 5$ \\
\hline Centre (Chambery) $(12)(+30)$ & 24 & 22.8 & 29.4 \\
\hline e $($ Besancon $)(16)(+1)$ & 25 & 22.5 & 29.0 \\
\hline Lirhe-Eco (Tse-Toulouse 1) (12)(+6o) & 26 & 22.2 & 28.6 \\
\hline Non-Gretha (Bordeaux 4) (17)(+29) & 27 & 19.9 & 25.6 \\
\hline Centre (Nantes) (17)(-11) & 28 & 19.7 & 25.4 \\
\hline Gael (Grenoble 2-Inra) (10)(+9) & 29 & 19.5 & 25.2 \\
\hline Clerse-Eco (Lille 1-Poly. Lille) $(23)(+1)$ & 30 & 19.4 & 25.1 \\
\hline Gredeg (Nice) $(34)(+4)$ & 31 & 19.4 & 25.0 \\
\hline e (Vers. St Quentin) $(17)(+18)$ & 32 & 19.0 & 24.5 \\
\hline $\operatorname{Leg}($ Dijon) $(32)(+19)$ & 33 & 18.6 & 24.0 \\
\hline Non-Cermes (Paris 11) $(8)(+20)$ & 34 & 18.6 & 24.0 \\
\hline$(-5)$ & 35 & 18.4 & 23.8 \\
\hline$(+4)$ & 36 & 18.3 & 23.6 \\
\hline$(27)(-9)$ & 37 & 18.0 & 23.2 \\
\hline Cent & 38 & $17 \cdot 7$ & 22.8 \\
\hline & 39 & 17.6 & 22.7 \\
\hline 3) $(-17)$ & 40 & 17.1 & 22.1 \\
\hline 4) $(26)(-17)$ & 41 & 17.0 & 21.9 \\
\hline 3) $(11)(-13)$ & 42 & 16.7 & 1.5 \\
\hline e 1$)(7)(-10)$ & 43 & 15.6 & 20.1 \\
\hline$(-7)$ & 44 & 15.6 & 20.1 \\
\hline Cen & $\begin{array}{l}44 \\
45\end{array}$ & 15.3 & 19.8 \\
\hline r 1-Inra) (17)(-29) & 46 & $15 \cdot 3$ & 19.7 \\
\hline$(17)(+19)$ & 47 & 14.6 & 18.9 \\
\hline ) $(35$ & 48 & $14 \cdot 3$ & \\
\hline Lille) $(18)(-28)$ & 49 & 13.8 & 17.8 \\
\hline ) $(10)(-1)$ & 50 & 13.8 & 17.8 \\
\hline$(-7)$ & 51 & 13.7 & 17.7 \\
\hline Inm $16(6)$ & 52 & 13.6 & 17.5 \\
\hline Les & 53 & $13 \cdot 3$ & 17.2 \\
\hline 3) & 54 & $\begin{array}{l}13.3 \\
13.1\end{array}$ & 16.8 \\
\hline es 1) $(25)(-8)$ & 55 & 12.7 & 16.4 \\
\hline & 56 & 12.4 & 16.0 \\
\hline$(+26)$ & 57 & 12.4 & 16.0 \\
\hline$(8)(-17)$ & 58 & 12.3 & 15.9 \\
\hline & 59 & 12.1 & 15.6 \\
\hline$(1,0 \pi)$ & 60 & 12.1 & 15.7 \\
\hline$(8)(-26)$ & 61 & 12.0 & 15.4 \\
\hline ira) $(17)(+14)$ & 62 & 12.0 & 15.5 \\
\hline & 63 & 11.8 & 15.3 \\
\hline$(-3)$ & 64 & 11.5 & 4.9 \\
\hline (7) $(-2)$ & 65 & 11.2 & 14.5 \\
\hline & 66 & 10.9 & 14.0 \\
\hline$(13)(+3)$ & 67 & 10.5 & 13.6 \\
\hline 3) $(6)(-11)$ & 68 & 10.4 & 13.4 \\
\hline & 69 & 10.2 & 13.2 \\
\hline & 70 & 10.2 & 13.2 \\
\hline$)(1$ & 71 & 10.2 & 13.2 \\
\hline$(+7)$ & 72 & 9.9 & 12.7 \\
\hline & 73 & 9.8 & 12.6 \\
\hline$(+16)$ & 74 & 9.8 & 12.6 \\
\hline & & 9.5 & 2.2 \\
\hline$(0)$ & 76 & 9.4 & 12.2 \\
\hline le) $(5)(-22)$ & & 9.3 & $12 . \mathrm{C}$ \\
\hline$(6)(+2)$ & 78 & 9.2 & 1.9 \\
\hline asb. 1) (7) & 79 & 9.1 & 11.7 \\
\hline$(13)(-21)$ & 80 & 8.2 & 10.6 \\
\hline & 81 & 8.2 & 10.6 \\
\hline 3) $(7)(-11)$ & 82 & & 9.9 \\
\hline 12) $(-34)$ & 83 & 7.6 & 9.7 \\
\hline & & $7 \cdot 5$ & 9.7 \\
\hline 121$)(-1)$ & & & \\
\hline$(7)(-8)$ & 86 & 5.6 & $.2=2$ \\
\hline (6)(o) & 87 & 4.9 & 6.3 \\
\hline$(5)(-4)$ & 88 & 4.6 & 5.9 \\
\hline Centre (Bretagne Sud) (7)(-6) & 89 & 3.9 & \\
\hline
\end{tabular}

\begin{tabular}{|c|c|c|c|}
\hline centre & rg. & p.c. & nor. \\
\hline Centre (Montpellier 3$)(6)(+4)$ & 21 & 3.18 & 33.7 \\
\hline Lef (Inra Nancy) (6)(+39) & 22 & 3.17 & 33.5 \\
\hline Gate (Lyon 2) (19) (+7) & 23 & 3.15 & $33 \cdot 4$ \\
\hline Centre (Perpignan) (8)(-8) & 24 & 2.95 & 31.3 \\
\hline Centre (Strasbourg 3) (11)(-9) & 25 & 2.95 & 31.3 \\
\hline Centre (Pau) (9)(+20) & 26 & 2.93 & 31.1 \\
\hline Beta (Nancy 2-Strasb. 1) (52)(+1) & 27 & 2.86 & 30.4 \\
\hline Centre (Paris 12) $(7)(+50)$ & 28 & 2.81 & 29.8 \\
\hline Gael (Grenoble 2-Inra) $(10)(+5)$ & 29 & 2.80 & 29.7 \\
\hline Ermes (Paris 2) (10)(-13) & 30 & 2.69 & 28.6 \\
\hline Lest-Eco (Aix Marseille 2-3) (9)(+57) & 31 & 2.68 & 28.5 \\
\hline Lameta (Montpellier 1-Inra) (17)(-9) & 32 & 2.58 & $27 \cdot 3$ \\
\hline Centre (Rennes 2) (6)(+30) & 33 & 2.57 & 27.2 \\
\hline Centre (Evry) $(16)(-1)$ & 34 & 2.50 & 26.5 \\
\hline Centre (La Rochelle) (5)(-14) & 35 & 2.48 & 26.3 \\
\hline Clerse-Eco (Lille 1-Poly. Lille) $(23)(+13)$ & 36 & 2.43 & 25.8 \\
\hline Leo (Orleans) (19)(o) & 37 & 2.38 & 25.2 \\
\hline Lepii (Grenoble 2-Inra) $(17)(+47)$ & 38 & 2.34 & 24.8 \\
\hline Cermes (Paris 11) (6)(+50) & 39 & 2.31 & $24 \cdot 5$ \\
\hline Aliss (Inra Ivry) $(13)(-22)$ & 40 & 2.29 & 24.2 \\
\hline Lirhe-Eco (Tse-Toulouse 1) (12) $(+47)$ & 41 & 2.29 & $24 \cdot 3$ \\
\hline Centre (Rouen) $(12)(+37)$ & 42 & 2.26 & 24.0 \\
\hline Mona-Tsv (Inra Ivry) (7) (+47) & 43 & 2.25 & 23.8 \\
\hline Non-Cepn (Paris 13) (7) $(+11)$ & 44 & 2.21 & 23.4 \\
\hline Centre (Besancon) (16)(-14) & 45 & 2.19 & 23.3 \\
\hline Centre (Mulhouse) (6)(-19) & 46 & 2.18 & 23.1 \\
\hline Centre (Paris 8) (13)(-8) & 47 & 2.18 & 23.1 \\
\hline Сepn (Paris 13) (18)(+16) & 48 & 2.17 & 23.0 \\
\hline Gretha (Bordeaux 4) (26)(-1) & 49 & 2.14 & 22.7 \\
\hline Centre (Nantes) (17)(-30) & 50 & 2.13 & 22.6 \\
\hline Crem (Caen-Rennes 1) (64)(-6) & 51 & 2.11 & 22.4 \\
\hline Non-Drm (Paris 9) $(27)(+2)$ & 52 & 2.10 & 22.2 \\
\hline Centre (Lille 2) (10)(-13) & 53 & 2.09 & 22.2 \\
\hline Cesaer (Inra Dijon) (8)(-25) & 54 & 2.04 & 21.6 \\
\hline Centre (St Etienne) (17)(-4) & 55 & 2.01 & 21.4 \\
\hline Centre (Toulon) (5)(-14) & 56 & 2.00 & 21.2 \\
\hline Centre (Vers. St Quentin) (17)(+9) & 57 & 1.92 & 20.3 \\
\hline Autre (Lille 1-Poly. Lille) (18)(-26) & 58 & 1.90 & 20.1 \\
\hline Centre (Reims) (19)(+1) & 59 & 1.85 & 19.6 \\
\hline Non-Ermes (Paris 2) (10)(-20) & 60 & 1.85 & 19.6 \\
\hline $\operatorname{Drm}$ (Paris 9) $(43)(+8)$ & 61 & 1.83 & 19.4 \\
\hline Centre (Tours) (6)(-26) & 62 & 1.79 & 19.0 \\
\hline Centre (Lille 3) (6)(-10) & 63 & 1.72 & 18.2 \\
\hline Non-Gretha $($ Bordeaux 4$)(17)(+4)$ & 64 & 1.70 & 18.0 \\
\hline Non-Gate (Lyon 2) (17)(+12) & 65 & 1.69 & $17 \cdot 9$ \\
\hline Centre (Metz) $(7)(-10)$ & 66 & 1.65 & $17 \cdot 5$ \\
\hline Gredeg (Nice) $(34)(+4)$ & 67 & 1.64 & $17 \cdot 4$ \\
\hline Centre (Marne La Vallee) (10)(-21) & 68 & 1.61 & 17.0 \\
\hline Centre (Le Havre) $(11)(+11)$ & 69 & 1.56 & 16.6 \\
\hline Cerag (Grenoble 2-Inra) (35)(+12) & 70 & 1.52 & 16.2 \\
\hline Autre (Pse-Paris 1) (13)(+1) & 71 & 1.45 & $15 \cdot 3$ \\
\hline Centre (Littoral) (12)(-22) & 72 & 1.44 & $15 \cdot 3$ \\
\hline Lem (Lille 1-Poly. Lille) $(70)(+2)$ & 73 & 1.37 & 14.5 \\
\hline $\operatorname{Leg}($ Dijon) $(32)(+10)$ & 74 & 1.33 & 14.1 \\
\hline Centre (La Reunion) (15)(-13) & 75 & 1.30 & 13.8 \\
\hline Centre (Limoges) (9)(-24) & 76 & 1.28 & 13.5 \\
\hline Centre (Paris 5) (6)(-34) & 77 & 1.26 & 13.4 \\
\hline Non-Beta (Nancy 2-Strasb. 1) (7)(-34) & 78 & 1.25 & $13 \cdot 3$ \\
\hline Autre (Grenoble 2-Inra) (13)(-38) & 79 & 1.23 & 13.1 \\
\hline Centre (Ant. Guy.) (13)(-21) & 80 & 1.05 & 11.2 \\
\hline Centre (Corte) $(6)(+2)$ & 81 & 1.03 & 10.9 \\
\hline Centre (Brest) (12)(-24) & 82 & 0.97 & 10.3 \\
\hline Non-Crem (Caen-Rennes 1) (25)(-7) & 83 & 0.94 & 10.0 \\
\hline Centre (Artois) (7)(-19) & 84 & 0.93 & 9.8 \\
\hline Centre (Lyon 1) (5)(-18) & 85 & 0.92 & 9.7 \\
\hline Centre (Angers) (14)(-13) & 86 & 0.91 & 9.7 \\
\hline Centre (Bretagne Sud) (7)(-17) & 87 & 0.78 & 8.2 \\
\hline Centre (Poitiers) (17)(-13) & 88 & 0.78 & 8.2 \\
\hline Autre (Aix Marseille 2-3) (25)(-3) & 89 & 0.76 & 8.1 \\
\hline
\end{tabular}


suite de la page précédente

\begin{tabular}{|c|c|c|c|c|c|}
\hline centre & rg. tot. & nor. & centre & rg. p.c. & nor. \\
\hline Centre (Amiens) (11)(-9) & $90 \quad 3.1$ & 4.0 & Centre (Amiens) (11)(-9) & $90 \quad 0.74$ & 7.8 \\
\hline
\end{tabular}

Notes : La colonne "rg." donne le rang, la colonne "tot." donne le score total, "nor." le score normalisé par rapport à celui du premier classé, "p.c." donne le score par chercheur. Entre les premières parenthèses se trouve le nombre de chercheurs, la variation de classement par rapport à $\mathrm{Clm}$ est donnée entre les deuxièmes parenthèses. Si cette variation est supérieure ou égale à 3, le nom est typographié en gras italique, si elle est inférieure ou égale à -3 , le nom est typographié en italique, sinon le nom est simplement typographié en gras.

\section{A.4 Universités, moins de 50 ans}

TABLE 28 - Universités, Moins de 50 ans, Citations totales, T=Dégressif

\begin{tabular}{|c|c|c|c|}
\hline université & rg. & tot. & nor. \\
\hline Pse-Paris $1(115)(+1)$ & 1 & 5169.2 & 100.0 \\
\hline Tse-Toulouse $1(74)(-1)$ & 2 & 4032.6 & 78.0 \\
\hline $\operatorname{Hec}(61)(+1)$ & 3 & 2188.3 & 42.3 \\
\hline Crest-Ensae $(30)(+1)$ & 4 & 1301.4 & 25.2 \\
\hline Aix Marseille 2-3 $(61)(-2)$ & 5 & 1260.4 & 24.4 \\
\hline Paris $10(46)(+2)$ & 6 & 861.8 & 16.7 \\
\hline Nancy 2-Strasbourg I (59)(-1) & 7 & 800.3 & $15 \cdot 5$ \\
\hline Lille 1-Polytech Lille $(111)(+1)$ & 8 & 747.8 & 14.5 \\
\hline Cergy Pontoise $(33)(-2)$ & 9 & 734.0 & 14.2 \\
\hline Ec. Polytechnique (19)(+1) & 10 & 664.4 & 12.9 \\
\hline Paris $9(70)(+2)$ & 11 & $593 \cdot 7$ & 11.5 \\
\hline Lyon $2(36)(+4)$ & 12 & 560.3 & 10.8 \\
\hline Caen-Rennes 1 (89)(-3) & 13 & $535 \cdot 3$ & 10.4 \\
\hline Grenoble 2-Inra $(74)(0)$ & 14 & 481.9 & $9 \cdot 3$ \\
\hline Inra Vers-Grig $(8)(+5)$ & 15 & 446.2 & 8.6 \\
\hline Bordeaux $4(43)(+1)$ & 16 & 363.0 & 7.0 \\
\hline Clermont i (19)(+2) & 17 & 361.8 & 7.0 \\
\hline Paris $2(19)(+3)$ & 18 & 310.4 & 6.0 \\
\hline Inra Rennes $(11)(+3)$ & 19 & 305.2 & 5.9 \\
\hline Besancon $(16)(+6)$ & 20 & $287 \cdot 3$ & 5.6 \\
\hline Dijon $(38)(+13)$ & 21 & 272.5 & $5 \cdot 3$ \\
\hline Cired $(7)(+14)$ & 22 & 243.6 & 4.7 \\
\hline Montpellier 1-Inra (26)(-11) & 23 & 232.6 & 4.5 \\
\hline Lille $2(10)(+13)$ & 24 & 231.6 & $4 \cdot 5$ \\
\hline Orleans $(21)(-1)$ & 25 & 213.0 & 4.1 \\
\hline Inra Ivry (19)(-3) & 26 & 212.2 & 4.1 \\
\hline Chambery (12)(+15) & 27 & 204.4 & 4.0 \\
\hline Nice $(38)(-3)$ & 28 & 201.9 & $3 \cdot 9$ \\
\hline Paris $11(13)(+10)$ & 29 & 195.7 & 3.8 \\
\hline Nantes $(17)(-12)$ & 30 & 186.1 & 3.6 \\
\hline Strasbourg $3(11)(-4)$ & 31 & 182.8 & $3 \cdot 5$ \\
\hline Le Mans (11)(-17) & 32 & 181.2 & $3 \cdot 5$ \\
\hline Versailles St Quentin $(17)(+7)$ & 33 & 176.6 & $3 \cdot 4$ \\
\hline Evry $(16)(-6)$ & 34 & 167.1 & 3.2 \\
\hline Paris $13(25)(-6)$ & 35 & 165.2 & 3.2 \\
\hline Pau (9)(+13) & 36 & 156.1 & 3.0 \\
\hline Reims (19)(-4) & 37 & 150.6 & 2.9 \\
\hline St Etienne $(17)(-7)$ & 38 & 141.0 & 2.7 \\
\hline Paris $8(13)(-7)$ & 39 & $127 \cdot 4$ & 2.5 \\
\hline Rouen (12) (+18) & 40 & 97.5 & 1.9 \\
\hline Inra Dijon (8)(-6) & 41 & 81.0 & 1.6 \\
\hline Paris $12(7)(+24)$ & 42 & 75.9 & 1.5 \\
\hline Perpignan $(8)(-13)$ & 43 & 74.8 & 1.5 \\
\hline La Rochelle $(5)(-1)$ & 44 & 69.9 & 1.4 \\
\hline Inra Nancy (6)(+18) & 45 & 68.4 & 1.3 \\
\hline Angers $(14)(+7)$ & 46 & 66.1 & 1.3 \\
\hline Montpellier $3(6)(-3)$ & 47 & 59.9 & 1.2 \\
\hline Limoges $(9)(+3)$ & 48 & $57 \cdot 5$ & 1.1 \\
\hline La Reunion (15)(-8) & 49 & 56.8 & 1.1 \\
\hline Rennes $2(6)(+11)$ & 50 & 53.6 & 1.0 \\
\hline Littoral (12)(-13) & 51 & $53 \cdot 5$ & 1.0 \\
\hline Mulhouse (6) $(-4)$ & 52 & 51.8 & 1.0 \\
\hline Marne La Vallee (10)(-8) & 53 & 51.5 & 1.0 \\
\hline Lille $3(6)(+3)$ & 54 & 46.2 & 0.9 \\
\hline
\end{tabular}

\begin{tabular}{|c|c|c|c|}
\hline université & rg. & p.c. & nor. \\
\hline Inra Vers-Grig $(8)(+2)$ & 1 & 55.78 & 100.0 \\
\hline Tse-Toulouse $1(74)(-1)$ & 2 & 54.64 & 98.0 \\
\hline Pse-Paris $1(115)(+1)$ & 3 & 44.83 & 80.4 \\
\hline Crest-Ensae $(30)(-2)$ & 4 & 43.67 & 78.3 \\
\hline Cired $(7)(+12)$ & 5 & 37.48 & 67.2 \\
\hline $\operatorname{Hec}(61)(+3)$ & 6 & 35.87 & $64 \cdot 3$ \\
\hline Ec. Polytechnique (19)(-2) & 7 & 34.25 & 61.4 \\
\hline Inra Rennes $(11)(+2)$ & 8 & $27 \cdot 74$ & $49 \cdot 7$ \\
\hline Lille $2(10)(+21)$ & 9 & 23.16 & 41.5 \\
\hline Cergy Pontoise $(33)(-3)$ & 10 & 22.24 & 39.9 \\
\hline Aix Marseille 2-3 (61)(-3) & 11 & 20.83 & $37 \cdot 4$ \\
\hline Clermont $1(19)(+7)$ & 12 & 19.04 & 34.1 \\
\hline Paris $10(46)(+2)$ & 13 & 18.72 & 33.6 \\
\hline Besancon $(16)(+10)$ & 14 & 17.96 & 32.2 \\
\hline Pau (9)(+19) & 15 & $17 \cdot 34$ & 31.1 \\
\hline Chambery $(12)(+27)$ & 16 & 17.04 & 30.5 \\
\hline Strasbourg $3(11)(-6)$ & 17 & 16.77 & 30.1 \\
\hline Paris $2(19)(+4)$ & 18 & 16.34 & $29 \cdot 3$ \\
\hline Le Mans (11)(-13) & 19 & 16.10 & 28.9 \\
\hline Lyon $2(36)(+13)$ & 20 & 15.61 & 28.0 \\
\hline Paris $11(13)(+20)$ & 21 & 15.06 & 27.0 \\
\hline La Rochelle (5) $(-8)$ & 22 & 13.98 & 25.1 \\
\hline Nancy 2-Strasbourg I (59)(o) & 23 & 13.68 & $24 \cdot 5$ \\
\hline Inra Nancy $(6)(+25)$ & 24 & 12.44 & 22.3 \\
\hline Paris $12(7)(+37)$ & 25 & 11.68 & 20.9 \\
\hline Nantes $(17)(-13)$ & 26 & 11.28 & 20.2 \\
\hline Inra Ivry (19)(-1) & 27 & 11.17 & 20.0 \\
\hline Montpellier $3(6)(-10)$ & 28 & 10.89 & $19 \cdot 5$ \\
\hline Evry $(16)(-4)$ & 29 & 10.78 & $19 \cdot 3$ \\
\hline Orleans $(21)(-2)$ & 30 & 10.39 & 18.6 \\
\hline Versailles St Quentin $(17)(+27)$ & 31 & 10.39 & 18.6 \\
\hline Paris $8(13)(-3)$ & 32 & 10.19 & 18.3 \\
\hline Inra Dijon (8)(-12) & 33 & 10.12 & 18.2 \\
\hline Mulhouse (6)(-14) & 34 & $9 \cdot 42$ & 16.9 \\
\hline Perpignan (8)(-23) & 35 & $9 \cdot 35$ & 16.8 \\
\hline Montpellier 1-Inra (26)(-20) & 36 & 8.95 & 16.0 \\
\hline Rennes $2(6)(+16)$ & 37 & 8.94 & 16.0 \\
\hline Bordeaux $4(43)(+7)$ & 38 & 8.54 & $15 \cdot 3$ \\
\hline Paris $9(70)(+13)$ & 39 & 8.48 & 15.2 \\
\hline Tours (6) $(-13)$ & 40 & 8.37 & 15.0 \\
\hline St Etienne $(17)(-4)$ & 41 & 8.30 & 14.9 \\
\hline Rouen $(12)(+21)$ & 42 & 8.12 & 14.6 \\
\hline Reims $(19)(+4)$ & 43 & $7 \cdot 92$ & 14.2 \\
\hline Lille $3(6)(-5)$ & 44 & $7 \cdot 70$ & 13.8 \\
\hline Dijon $(38)(+21)$ & 45 & 7.27 & 13.0 \\
\hline Toulon (5)(-15) & 46 & 7.17 & 12.9 \\
\hline Limoges (9)(-9) & 47 & 6.77 & 12.1 \\
\hline Lille 1-Polytech Lille (III)(o) & 48 & 6.74 & 12.1 \\
\hline Paris $13(25)(+1)$ & 49 & 6.61 & 11.9 \\
\hline Grenoble 2-Inra $(74)(+6)$ & 50 & 6.51 & 11.7 \\
\hline Caen-Rennes I (89)(-11) & 51 & 6.01 & 10.8 \\
\hline & 52 & $5 \cdot 79$ & 10.4 \\
\hline Marne La Vallee (10)(-18) & 53 & $5 \cdot 42$ & 9.7 \\
\hline Nice $(38)(0)$ & 54 & $5 \cdot 31$ & $9 \cdot 5$ \\
\hline
\end{tabular}


suite de la page précédente

\begin{tabular}{|c|c|c|c|c|c|c|c|}
\hline université & rg. & tot. & nor. & université & rg. & p.c. & nor. \\
\hline Poitiers (17)(-4) & 55 & 46.2 & 0.9 & Paris 5 (6)(-23) & 55 & 5.12 & 9.2 \\
\hline Tours $(6)(-4)$ & 56 & 46.0 & 0.9 & Lyon $1(5)(+2)$ & 56 & 4.91 & 8.8 \\
\hline Antilles Guyane (13)(-11) & 57 & 41.4 & 0.8 & Angers $(14)(+3)$ & 57 & 4.72 & 8.5 \\
\hline Le Havre $(11)(+2)$ & 58 & 40.2 & 0.8 & Littoral (12)(-22) & 58 & 4.45 & 8.0 \\
\hline $\operatorname{Metz}(7)(-3)$ & 59 & 37.6 & 0.7 & La Reunion (15)(-8) & 59 & 3.79 & 6.8 \\
\hline Toulon $(5)(-5)$ & 60 & 35.9 & 0.7 & Le Havre (11)(+4) & 60 & 3.66 & 6.6 \\
\hline Brest (12)(-14) & 61 & 30.6 & 0.6 & Antilles Guyane (13)(-15) & 61 & $3 \cdot 31$ & 5.9 \\
\hline Paris $5(6)(-8)$ & 62 & 28.2 & 0.5 & Poitiers $(17)(-1)$ & 62 & 2.72 & 4.9 \\
\hline Lyon $1(5)(+2)$ & 63 & 24.5 & 0.5 & Brest (12)(-19) & 63 & 2.66 & 4.8 \\
\hline Artois (7) $(-5)$ & 64 & 17.9 & 0.4 & Artois $(7)(-9)$ & 64 & 2.56 & 4.6 \\
\hline Amiens (11)(-3) & 65 & 16.8 & 0.3 & Corte $(6)(+2)$ & 65 & 2.33 & 4.2 \\
\hline Corte $(6)(+1)$ & 66 & 14.0 & 0.3 & Amiens (11)(-1) & 66 & 1.53 & 2.7 \\
\hline Bretagne Sud (7)(-3) & 67 & 10.3 & 0.2 & Bretagne Sud (7)(-8) & 67 & 1.48 & 2.7 \\
\hline
\end{tabular}

Notes : La colonne "rg." donne le rang, la colonne "tot." donne le score total, "nor." le score normalisé par rapport à celui du premier classé, "p.c." donne le score par chercheur. Entre les premières parenthèses se trouve le nombre de chercheurs, la variation de classement par rapport à Clm est donnée entre les deuxièmes parenthèses. Si cette variation est supérieure ou égale à 3, le nom est typographié en gras italique, si elle est inférieure ou égale à -3 , le nom est typographié en italique, sinon le nom est simplement typographié en gras.

TABlE 29 - Universités, Moins de 50 ans, Indice G, T=Dégressif

\begin{tabular}{|c|c|c|c|}
\hline université & rg. & tot. & nor. \\
\hline Tse-Toulouse $\mathbf{I}(74)(0)$ & 1 & 72.2 & 100.0 \\
\hline Pse-Paris $1(115)(0)$ & 2 & 69.4 & 96.2 \\
\hline $\operatorname{Hec}(61)(+1)$ & 3 & 66.6 & 92.2 \\
\hline Aix Marseille 2-3 (61)(-1) & 4 & 46.4 & $64 \cdot 3$ \\
\hline Paris $10(46)(+3)$ & 5 & 41.6 & 57.6 \\
\hline Nancy 2-Strasbourg 1 (59)(o) & 6 & 40.3 & 55.8 \\
\hline Crest-Ensae $(30)(-2)$ & 7 & 39.1 & 54.2 \\
\hline Ec. Polytechnique (19) $(+3)$ & 8 & 38.8 & $53 \cdot 7$ \\
\hline Lyon $2(36)(+7)$ & 9 & 33.9 & 47.0 \\
\hline Inra Vers-Grig $(8)(+10)$ & 10 & 32.6 & 45.2 \\
\hline Cergy Pontoise (33)(-4) & 11 & 31.9 & 44.2 \\
\hline Paris $2(19)(+9)$ & 12 & 31.2 & 43.2 \\
\hline Le Mans $(11)(+2)$ & 13 & 30.4 & 42.1 \\
\hline Caen-Rennes 1 (89)(-4) & 14 & 30.0 & 41.6 \\
\hline Lille 1-Polytech Lille (111)(-5) & 15 & 30.0 & 41.6 \\
\hline Paris $9(70)(-3)$ & 16 & 29.2 & 40.5 \\
\hline Bordeaux 4 (43)(o) & 17 & 25.1 & 34.8 \\
\hline Inra Rennes (11)(+5) & 18 & 25.1 & 34.8 \\
\hline Grenoble 2-Inra $(74)(-5)$ & 19 & 24.6 & 34.1 \\
\hline Cired $(7)(+16)$ & 20 & 24.2 & 33.6 \\
\hline Clermont 1 (19)(-2) & 21 & 23.8 & 33.0 \\
\hline Lille $2(10)(+15)$ & 22 & 22.9 & 31.8 \\
\hline Chambery (12)(+19) & 23 & 22.8 & 31.6 \\
\hline Besancon $(16)(+2)$ & 24 & 22.5 & 31.1 \\
\hline Dijon $(38)(+9)$ & 25 & 21.3 & 29.6 \\
\hline Inra Ivry $(19)(-3)$ & 26 & 20.6 & 28.6 \\
\hline Paris $11(13)(+12)$ & 27 & 20.5 & 28.4 \\
\hline Montpellier 1-Inra (26)(-16) & 28 & 20.2 & 28.0 \\
\hline Nice $(38)(-3)$ & 29 & 20.2 & 28.0 \\
\hline Nantes $(17)(-12)$ & 30 & 19.7 & $27 \cdot 3$ \\
\hline Versailles St Quentin (17)(+9) & 31 & 19.0 & 26.3 \\
\hline Evry $(16)(-4)$ & 32 & 18.4 & $25 \cdot 5$ \\
\hline Reims (19)(o) & 33 & 18.3 & $25 \cdot 4$ \\
\hline Pau (9)(+15) & 34 & 17.7 & 24.6 \\
\hline Orleans $(21)(-11)$ & & $17 \cdot 3$ & 24.0 \\
\hline Strasbourg $3(11)(-9)$ & 36 & 16.7 & 23.1 \\
\hline St Etienne (17)(-6) & 37 & 15.6 & 21.6 \\
\hline Angers $(14)(+15)$ & 38 & $15 \cdot 3$ & 21.2 \\
\hline Paris $13(25)(-10)$ & 39 & 14.7 & 20.3 \\
\hline Rouen $(12)(+18)$ & 40 & 13.1 & 18.1 \\
\hline Inra Nancy $(6)(+22)$ & 41 & 12.4 & 17.2 \\
\hline Paris $8(13)(-9)$ & 42 & 12.4 & 17.2 \\
\hline Inra Dijon (8)(-8) & 43 & 12.3 & 17.1 \\
\hline
\end{tabular}

\begin{tabular}{|c|c|c|c|}
\hline université & rg. & p.c. & nor. \\
\hline Cired $(7)(+16)$ & 1 & 6.24 & 100.0 \\
\hline Inra Vers-Grig $(8)(+1)$ & 2 & 6.08 & $97 \cdot 4$ \\
\hline Tse-Toulouse 1 (74)(-2) & 3 & $5 \cdot 30$ & 84.8 \\
\hline Inra Rennes $(11)(+6)$ & 4 & 4.61 & 73.9 \\
\hline Pse-Paris $1(115)(-1)$ & 5 & 4.58 & $73 \cdot 3$ \\
\hline Crest-Ensae (30)(-4) & 6 & 4.53 & 72.5 \\
\hline Ec. Polytechnique $(19)(-2)$ & 7 & $4 \cdot 38$ & 70.1 \\
\hline Chambery $(12)(+35)$ & 8 & 3.83 & 61.3 \\
\hline Paris $10(46)(+6)$ & 9 & 3.51 & 56.2 \\
\hline Cergy Pontoise (33)(-3) & 10 & 3.49 & 55.9 \\
\hline Le Mans $(11)(-5)$ & 11 & 3.40 & $54 \cdot 5$ \\
\hline Hec $(61)(-3)$ & 12 & 3.31 & 53.0 \\
\hline Montpellier $3(6)(+5)$ & 13 & 3.18 & 51.0 \\
\hline Inra Nancy (6) (+35) & 14 & 3.17 & 50.7 \\
\hline Clermont $1(19)(+4)$ & 15 & 3.11 & 49.9 \\
\hline Paris $11(13)(+25)$ & 16 & 3.00 & 48.1 \\
\hline Aix Marseille 2-3 (61)(-9) & 17 & 2.97 & $47 \cdot 5$ \\
\hline Perpignan (8)(-6) & 18 & 2.95 & $47 \cdot 3$ \\
\hline Strasbourg $3(11)(-7)$ & 19 & 2.95 & $47 \cdot 3$ \\
\hline$P a u(9)(+14)$ & 20 & 2.93 & 47.0 \\
\hline Montpellier 1-Inra (26)(-5) & 21 & 2.84 & $45 \cdot 5$ \\
\hline Paris $12(7)(+40)$ & 22 & 2.81 & 45.0 \\
\hline Nancy 2-Śtrasbourg 1 (59)(o) & 23 & 2.68 & 43.0 \\
\hline Rennes $2(6)(+29)$ & 24 & 2.57 & 41.1 \\
\hline Evry (16)(o) & 25 & 2.50 & 40.0 \\
\hline La Rochelle (5)(-12) & 26 & 2.48 & 39.7 \\
\hline Lyon $2(36)(+7)$ & 27 & 2.48 & 39.7 \\
\hline Inra Ivry (19)(-2) & 28 & 2.27 & 36.4 \\
\hline Paris $2(19)(-6)$ & 29 & 2.27 & 36.4 \\
\hline Rouen $(12)(+33)$ & 30 & 2.26 & 36.2 \\
\hline Orleans $(21)(-3)$ & 31 & 2.25 & 36.1 \\
\hline Besancon $(16)(-8)$ & 32 & 2.19 & 35.2 \\
\hline Mulhouse (6)(-13) & 33 & 2.18 & 34.9 \\
\hline Paris $13(25)(+17)$ & 34 & 2.18 & 34.9 \\
\hline Paris $8(13)(-4)$ & 35 & 2.18 & 35.0 \\
\hline Nantes (17)(-23) & 36 & 2.13 & 34.2 \\
\hline Lille $2(10)(-7)$ & 37 & 2.09 & $33 \cdot 5$ \\
\hline Inra Dijon (8)(-17) & 38 & 2.04 & 32.6 \\
\hline St Etienne $(17)(-2)$ & 39 & 2.01 & 32.3 \\
\hline Toulon (5)(-9) & 40 & 2.00 & 32.0 \\
\hline Bordeaux $4(43)(+4)$ & 41 & 1.97 & 31.5 \\
\hline Paris $9(70)(+10)$ & 42 & 1.93 & 31.0 \\
\hline Versailles St Quentin $(17)(+14)$ & & & \\
\hline & \multicolumn{3}{|c|}{ suite page suivante } \\
\hline
\end{tabular}


suite de la page précédente

\begin{tabular}{|c|c|c|c|}
\hline université & rg. & tot. & nor. \\
\hline Paris $12(7)(+22)$ & 44 & 12.1 & 16.8 \\
\hline Perpignan (8)(-15) & 45 & 12.0 & 16.6 \\
\hline Limoges $(9)(+5)$ & 46 & 11.8 & 16.4 \\
\hline Mulhouse $(6)(+1)$ & 47 & 11.5 & 16.0 \\
\hline Poitiers $(17)(+2)$ & 48 & 11.2 & $15 \cdot 5$ \\
\hline Tours $(6)(+3)$ & 49 & 10.9 & 15.0 \\
\hline Montpellier $3(6)(-6)$ & 50 & 10.4 & 14.4 \\
\hline La Reunion (15)(-10) & 51 & 10.2 & 14.2 \\
\hline Marne La Vallee (10)(-6) & 52 & 10.2 & 14.1 \\
\hline Le Havre $(11)(+7)$ & 53 & 9.9 & 13.7 \\
\hline Toulon $(5)(+1)$ & 54 & 9.8 & 13.5 \\
\hline $\operatorname{Metz}(7)(+1)$ & 55 & $9 \cdot 5$ & 13.1 \\
\hline Lille $3(6)(+1)$ & 56 & 9.4 & 13.1 \\
\hline La Rochelle (5)(-14) & 57 & 9.3 & 12.9 \\
\hline Rennes $2(6)(+3)$ & 58 & 9.2 & 12.8 \\
\hline Antilles Guyane (13)(-13) & 59 & 8.2 & 11.4 \\
\hline Brest (12)(-12) & 60 & 8.2 & 11.4 \\
\hline Littoral (12)(-23) & 61 & 7.6 & 10.5 \\
\hline Paris $5(6)(-8)$ & 62 & $7 \cdot 5$ & 10.4 \\
\hline Artois $(7)(-4)$ & 63 & 5.6 & 7.8 \\
\hline Corte $(6)(+3)$ & 64 & 4.9 & 6.8 \\
\hline Lyon $1(5)(0)$ & 65 & 4.6 & 6.4 \\
\hline Bretagne Sud $(7)(-2)$ & 66 & 3.9 & 5.5 \\
\hline Amiens $(11)(-5)$ & 67 & 3.1 & $4 \cdot 3$ \\
\hline
\end{tabular}

\begin{tabular}{|c|c|c|}
\hline université & rg. p.c. & nor. \\
\hline Reims $(19)(+3)$ & $44 \quad 1.85$ & 29.7 \\
\hline Grenoble 2-Inra $(74)(+11)$ & $45 \quad 1.83$ & 29.4 \\
\hline Tours (6)(-19) & $46 \quad 1.79$ & 28.7 \\
\hline Caen-Rennes I (89)(-7) & $\begin{array}{ll}47 & 1.78\end{array}$ & 28.6 \\
\hline Lille $3(6)(-9)$ & $48 \quad 1.72$ & $27 \cdot 5$ \\
\hline Nice $(38)(+5)$ & 491.71 & 27.4 \\
\hline Lille 1-Polytech Lille (111)(-2) & $50 \quad 1.68$ & 26.9 \\
\hline Metz (7)(-9) & $51 \quad 1.65$ & 26.4 \\
\hline Marne La Vallee (10)(-17) & 521.61 & 25.7 \\
\hline Le Havre $(11)(+11)$ & $53 \quad 1.56$ & 25.0 \\
\hline Dijon $(38)(+12)$ & $54 \quad 1.49$ & 23.9 \\
\hline Littoral (12)(-19) & $55 \quad 1.44$ & 23.1 \\
\hline La Reunion (15)(-5) & $56 \quad 1.30$ & 20.9 \\
\hline Limoges (9)(-19) & $57 \quad 1.28$ & 20.4 \\
\hline Paris $5(6)(-26)$ & $58 \quad 1.26$ & 20.3 \\
\hline Antilles Guyane (13)(-13) & 591.05 & 16.9 \\
\hline Corte $(6)(+7)$ & $60 \quad 1.03$ & 16.4 \\
\hline Brest (12)(-17) & 610.97 & 15.5 \\
\hline Artois (7) $(-7)$ & $\begin{array}{lll}62 & 0.93\end{array}$ & 14.9 \\
\hline Lyon 1 (5)(-5) & $\begin{array}{ll}63 & 0.92\end{array}$ & 14.7 \\
\hline Angers (14)(-4) & 640.91 & 14.6 \\
\hline Bretagne Sud (7)(-6) & 650.78 & 12.4 \\
\hline Poitiers (17)(-4) & $\begin{array}{ll}66 & 0.78\end{array}$ & 12.5 \\
\hline Amiens (11) $(-2)$ & 670.74 & 11.9 \\
\hline
\end{tabular}

Notes : La colonne "rg." donne le rang, la colonne "tot." donne le score total, "nor." le score normalisé par rapport à celui du premier classé, "p.c." donne le score par chercheur. Entre les premières parenthèses se trouve le nombre de chercheurs, la variation de classement par rapport à Clm est donnée entre les deuxièmes parenthèses. Si cette variation est supérieure ou égale à 3, le nom est typographié en gras italique, si elle est inférieure ou égale à -3 , le nom est typographié en italique, sinon le nom est simplement typographié en gras.

\section{B Classements ne gardant que les 10 ou 30 chercheurs les plus productifs}

\section{B.1 Centres}

TABLE 30 - Centres, 10 ou 30 plus productifs, Citations totales, T=Dégressif

\begin{tabular}{lrrr}
\hline \hline centre (top 1o) & rg. & p.c. & nor. \\
\hline Gremaq (Tse-Toulouse 1)(o) & 1 & 605.2 & 100.0 \\
Pjse (Pse-Paris 1)(o) & 2 & 405.4 & 67.0 \\
Ces (Pse-Paris 1)(+2) & 3 & 205.6 & 34.0 \\
Non-Grecsta (Crest-Ensae)(+5) & 4 & 158.5 & 26.2 \\
Greghec (Hec)(-1) & 5 & 158.2 & 26.2 \\
Greqam (Aix Marseille 2-3)(-3) & 6 & 135.1 & 22.3 \\
Grecsta (Crest-Ensae)(+1) & 7 & 134.1 & 22.2 \\
Lerna (Tse-Toulouse 1)(-1) & 8 & 122.6 & 20.3 \\
Centre (Iep Paris)(+3) & 9 & 101.2 & 16.7 \\
Preg (Ec. Polytechnique)(-4) & 10 & 96.2 & 15.9 \\
Economix (Paris 10)(+2) & 11 & 80.7 & 13.3 \\
Drm (Paris 9)(+14) & 12 & 78.7 & 13.0 \\
Beta (Nancy 2-Strasb. 1)(-2) & 13 & 76.9 & 12.7 \\
Lameta (Montpellier 1-Inra)(+1) & 14 & 69.3 & 11.5 \\
Thema (Cergy Pontoise)(-5) & 15 & 59.6 & 9.9 \\
Eco. Pub. (Inra Vers-Grig)(+3) & 16 & 51.4 & 8.5 \\
Gredeg (Nice)(+8) & 17 & 48.8 & 8.1 \\
Gate (Lyon 2)(+6) & 18 & 46.1 & 7.6 \\
Cerdi (Clermont 1)(+9) & 19 & 45.2 & $7 \cdot 5$ \\
Centre (Cired)(+15) & 20 & 44.7 & 7.4 \\
Leg (Dijon)(+31) & 21 & 40.5 & 6.7 \\
Centre (Besancon)(-1) & 22 & 38.5 & 6.4 \\
Cepn (Paris 13)(+13) & 23 & 37.5 & 6.2 \\
Autre (Tse-Toulouse 1)(-8) & 24 & 36.7 & 6.1 \\
\hline
\end{tabular}

\begin{tabular}{lrrr}
\hline \hline centre (top 30) & rg. & p.c. & nor. \\
\hline Gremaq (Tse-Toulouse 1)(o) & 1 & $243 \cdot 3$ & 100.0 \\
Pjse (Pse-Paris 1)(o) & 2 & 177.4 & 72.9 \\
Ces (Pse-Paris 1)(o) & 3 & 104.9 & 43.1 \\
Centre (Iep Paris)(+3) & 4 & 101.2 & 41.6 \\
Greghec (Hec)(+1) & 5 & 85.2 & 35.0 \\
Lerna (Tse-Toulouse 1)(-1) & 6 & 70.1 & 28.8 \\
Greqam (Aix Marseille 2-3)(-3) & 7 & 62.2 & 25.6 \\
Non-Grecsta (Crest-Ensae)(+6) & 8 & 59.2 & 24.4 \\
Grecsta (Crest-Ensae)(-1) & 9 & 57.0 & 23.4 \\
Eco. Pub. (Inra Vers-Grig)(+5) & 10 & 43.1 & 17.7 \\
Preg (Ec. Polytechnique)(-2) & 11 & 41.4 & 17.0 \\
Beta (Nancy 2-Strasb. 1)(-2) & 12 & 37.2 & 15.3 \\
Economix (Paris 10)(-2) & 13 & 36.7 & 15.1 \\
Drm (Paris 9)(+25) & 14 & 34.5 & 14.2 \\
Centre (Ens Cachan)(-4) & 15 & 34.3 & 14.1 \\
Centre (Cired)(+5) & 16 & 33.4 & 13.7 \\
Smart (Inra Rennes)(-1) & 17 & 28.6 & 11.8 \\
Thema (Cergy Pontoise)(-7) & 18 & 26.2 & 10.8 \\
Lameta (Montpellier 1-Inra)(+11) & 19 & 24.6 & 10.1 \\
Centre (Strasbourg 3)(o) & 20 & 22.0 & 9.1 \\
Cerdi (Clermont 1)(+13) & 21 & 20.6 & 8.5 \\
Gredeg (Nice)(+11) & 22 & 20.3 & 8.4 \\
Gate (Lyon 2)(+2) & 23 & 19.5 & 8.0 \\
Gael (Grenoble 2-Inra)(+7) & 24 & 18.9 & 7.8 \\
\hline & suite page suivante \\
\hline
\end{tabular}


suite de la page précédente

\begin{tabular}{|c|c|c|c|}
\hline centre (top 10) & rg. & p.c. & \\
\hline Centre (Ens Cachan) $(-2)$ & 25 & & \\
\hline Gretha $($ Bordeaux 4$)(+4)$ & 26 & & \\
\hline Smart (Inra Rennes)(+2) & 27 & 33.1 & \\
\hline Crem (Caen-Rennes I)(-11) & 28 & 32.1 & \\
\hline Centre (Vers. St Quentin) $(+23)$ & 29 & 30.9 & \\
\hline Non-Gate $($ Lyon 2$)(+21)$ & 30 & 29.9 & \\
\hline Clerse-Eco (Lille 1-Poly. Lille) $(+13)$ & 31 & 29.7 & \\
\hline Non-Drm (Paris 9)(-13) & 32 & 29.7 & \\
\hline Ermes (Paris 2$)(-1)$ & 33 & 28.3 & \\
\hline Centre (Strasbourg 3)(-2) & 34 & 28.0 & \\
\hline Non-Cermes (Paris 11) $(+28)$ & 35 & 27.1 & \\
\hline Centre (Nantes)(-16) & 36 & 26.8 & \\
\hline Gael (Grenoble 2-Inra)(+1) & 37 & 26.6 & \\
\hline Non-Gretha $($ Bordeaux 4$)(+3)$ & 38 & 26.0 & \\
\hline Cerag (Grenoble 2-Inra) $(+21)$ & 39 & 24.9 & \\
\hline Lirhe-Eco (Tse-Toulouse 1) $(+47)$ & 40 & 23.6 & \\
\hline Centre (Lille 2$)(+16)$ & 41 & $23 \cdot 3$ & \\
\hline Lem (Lille 1-Poly. Lille)(-20) & 42 & 23.2 & \\
\hline Cermes (Paris 11) $(+56)$ & 43 & 21.5 & \\
\hline Iredu-Ec & 44 & 21.0 & \\
\hline Leo (Orleans) $(-10)$ & 45 & 21.0 & \\
\hline Centre (Chambery) $(+22)$ & 46 & 20.2 & \\
\hline Centre (Le Mans) $(-32)$ & 47 & 20.2 & \\
\hline Centre $(\mathrm{Pau})(+10)$ & 48 & 20.2 & \\
\hline Lepii (Grenoble 2-Inra) $(+31)$ & 49 & 20.1 & \\
\hline Autre (Pse-Paris 1$)(+21)$ & 50 & 18.3 & \\
\hline Centre (Reim & 51 & 17.8 & \\
\hline Aliss (Inra Ivry) $(-25)$ & 52 & 17.1 & \\
\hline Autre (Aix Marseille 2-3)(+6) & 53 & 17.0 & \\
\hline Centre (Paris 8)(-12) & 54 & 16.7 & \\
\hline$($ Inra Ivry $)(+46)$ & 55 & 16.5 & \\
\hline$(-19)$ & 56 & 16.2 & \\
\hline Autre (Lille 1-Poly. Lille)(-26) & 57 & 16.1 & \\
\hline Non-Ermes (Paris 2)(-19) & 58 & 14.4 & \\
\hline é) $(-19)$ & 59 & 14.0 & \\
\hline Centre & 60 & 13.7 & \\
\hline Lef (Inra Nanc & 61 & 12.9 & \\
\hline Centre (Paris 12)(+12) & 62 & 12.6 & \\
\hline Cesaer (Inra Dijon)(-19) & 63 & 12.6 & \\
\hline Lest-Eco (Aix Marseille 2-3)(+29) & 64 & 12.3 & \\
\hline Centre (Paris 7$)(+31)$ & 65 & 11.5 & \\
\hline Rennes 1)(-2) & 66 & 10.5 & \\
\hline ellier 1-Inra)(-19) & 67 & 10.4 & \\
\hline Centre (Rou & 68 & 10.4 & \\
\hline Centre & 69 & 9.6 & \\
\hline ble 2-Inra) $(-25)$ & 70 & 9.1 & \\
\hline Centre (Lille & 71 & 8.8 & \\
\hline Centre (La R & 72 & 8.6 & \\
\hline er 1 -Inra)(o) & 73 & 8.6 & \\
\hline Centre (Perp & 74 & 8.5 & \\
\hline Centre (Rennes 2) $(+9)$ & 75 & $7 \cdot 7$ & \\
\hline Non-Beta (Nancy 2-Strasb. 1)(+4) & 76 & $7 \cdot 7$ & \\
\hline Non-Cepn $($ Paris 13) $(+4)$ & 77 & $7 \cdot 5$ & \\
\hline Centre (Montpellier 3)(-9) & 78 & $7 \cdot 4$ & \\
\hline Centre $($ Angers $)(+3)$ & 79 & $7 \cdot 3$ & \\
\hline Centre (Valenciennes) $(+11)$ & 80 & 6.4 & \\
\hline & 81 & 6.3 & \\
\hline Non-Gredeg (Nice)(-7) & 82 & 6.3 & \\
\hline Centre (Mulhouse) $(-17)$ & 83 & 6.2 & \\
\hline Centre (Cnam)(-11) & 84 & 6.1 & \\
\hline Autre (Dijon) $(+12)$ & 85 & 5.9 & \\
\hline Centre (Tou & 86 & 5.9 & \\
\hline Centre (Poitiers) $(-32)$ & 87 & 5.8 & \\
\hline Centre (Toulouse 2$)(+15)$ & 88 & $5 \cdot 5$ & \\
\hline & 89 & & \\
\hline Centre (Marne La Vallee)(-20) & 90 & $5 \cdot 3$ & \\
\hline Centre (Brest)(-25) & 91 & 5.0 & \\
\hline Centre (Ant. Guy.)(-27) & 92 & 4.9 & \\
\hline
\end{tabular}

centre (top 30) rg. p.c. nor.

\begin{tabular}{lllll}
\hline Centre (Lille 2) $(+22)$ & 25 & 18.0 & $7 \cdot 4$
\end{tabular}

$\begin{array}{llll}\text { Iredu-Eco (Dijon) }(+59) & 26 & 17.7 & 7 \cdot 3\end{array}$

Non-Cermes (Paris 11) (+31) $\quad 27 \quad 17.6 \quad 7.2$

$\begin{array}{llll}\text { Ermes (Paris 2)(o) } & 28 & 17.5 & 7.2\end{array}$

Crem (Caen-Rennes 1)(-11) 29 17.0 7.0

$\begin{array}{llll}\text { Centre (Besancon) }(-1) & 30 & 16.8 & 6.9\end{array}$

$\begin{array}{llll}\text { Autre (Tse-Toulouse 1)(-7) } & 31 & 16.4 & 6.7\end{array}$

$\begin{array}{llll}\operatorname{Leg}(\text { Dijon })(+40) & 32 & 15.8 & 6.5\end{array}$

$\begin{array}{llll}\text { Cepn }(\text { Paris 13) }(+13) & 33 & 14.8 & 6.1\end{array}$

Non-Drm (Paris 9)(-6) $\quad 34 \quad 14.8 \quad 6.1$

$\begin{array}{llll}\text { Gretha (Bordeaux 4) }(+1) & 35 & 14.4 & 5.9\end{array}$

$\begin{array}{llll}\text { Centre (La Rochelle)(-17) } & 36 & 14.0 & 5.8\end{array}$

$\begin{array}{llll}\text { Centre (Chambery) }(+26) & 37 & 13.8 & 5.7\end{array}$

Centre (Vers. St Quentin)(+32) $38 \quad 13.3 \quad 5.5$

$\begin{array}{llll}\text { Lef (Inra Nancy) }(+17) & 39 & 12.9 & 5.3\end{array}$

$\begin{array}{llll}\text { Centre (Nantes)(-17) } & 40 & 12.8 & 5 \cdot 3\end{array}$

Lem (Lille 1-Poly. Lille)(-3) $\quad 41 \quad 12.8 \quad 5.3$

Lirhe-Eco (Tse-Toulouse 1)(+50) $42 \quad 12.6 \quad 5.2$

$\begin{array}{llll}\text { Cermes (Paris 11) }(+58) & 43 & 12.4 & 5.1\end{array}$

Centre $($ Pau $)(+13) \quad 44 \quad 12.3 \quad 5.0$

Clerse-Eco (Lille 1-Poly. Lille)(+7) $45 \quad 11.7 \quad 4.8$

$\begin{array}{llll}\text { Centre }(\text { Paris } 7)(+41) & 46 & 11.5 & 4.7\end{array}$

$\begin{array}{llll}\text { Cerag (Grenoble 2-Inra) }(+34) \quad 47 & 11.5 & 4.7\end{array}$

$\begin{array}{llll}\text { Cesaer (Inra Dijon)(-27) } & 48 & 11.4 & 4.7\end{array}$

$\begin{array}{llll}\text { Non-Gate (Lyon 2)(+24) } & 49 & 11.4 & 4.7\end{array}$

$\begin{array}{llll}\text { Centre (Le Mans)(-33) } & 50 & 11.2 & 4.6\end{array}$

Lest-Eco (Aix Marseille 2-3)(+36) $\quad 51 \quad 9.8 \quad 4.0$

Aliss (Inra Ivry)(-26) $\quad 52 \quad 9.6 \quad 3.9$

$\begin{array}{llll}\text { Centre (Evry)(-18) } & 53 & 9.4 & 3.9\end{array}$

$\begin{array}{llll}\text { Non-Gretha }(\text { Bordeaux } 4)(+5) \quad 54 & 9.4 & 3.9\end{array}$

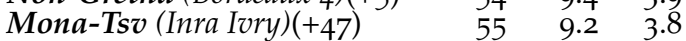

Moisa (Montpellier 1-Inra)(-6) $\quad 56 \quad 8.6 \quad 3.5$

Lepii (Grenoble 2-Inra) (+35) $\quad 57 \quad 8.1 \quad 3 \cdot 3$

Centre (Lille 3)(-20) $\quad 58 \quad 8.0 \quad 3 \cdot 3$

$\begin{array}{llll}\text { Leo (Orleans)(-11) } & 59 & 7.9 & 3.2\end{array}$

$\begin{array}{llll}\text { Centre (Rennes 2) }(+8) & 60 & 7.7 & 3.2\end{array}$

$\begin{array}{llll}\text { Centre (Montpellier 3)(-19) } & 61 & 7.4 & 3.1\end{array}$

$\begin{array}{llll}\text { Centre (Perpignan)(-29) } & 62 & 7.4 & 3.1\end{array}$

$\begin{array}{llll}\text { Centre (Paris 8)(-11) } & 63 & 6.9 & 2.8\end{array}$

$\begin{array}{llll}\text { Non-Ermes (Paris 2)(-19) } & 64 & 6.8 & 2.8\end{array}$

$\begin{array}{llll}\text { Autre (Aix Marseille 2-3) }(+17) & 65 & 6.7 & 2.8\end{array}$

$\begin{array}{llll}\text { Centre (Reims)(o) } & 66 & 6.6 & 2.7\end{array}$

$\begin{array}{llll}\text { Centre (St Etienne)(-8) } & 67 & 6.6 & 2.7\end{array}$

Autre (Pse-Paris 1)(+17) $\quad 68 \quad 6.4 \quad 2.6$

$\begin{array}{lllll}\text { Centre (Valenciennes) }(+10) & 69 & 6.4 & 2.6\end{array}$

$\begin{array}{llll}\text { Non-Cepn (Paris 13) }(+1) & 70 & 6.3 & 2.6\end{array}$

$\begin{array}{llll}\text { Centre (Mulhouse) }(-31) & 71 & 6.2 & 2.5\end{array}$

$\begin{array}{llll}\text { Centre (Cnam)(-21) } & 72 & 6.1 & 2.5\end{array}$

Autre (Lille 1-Poly. Lille)(-32) $\quad \begin{array}{llll}73 & 5.9 & 2.4\end{array}$

$\begin{array}{llll}\text { Centre (Toulon)(-13) } & 74 & 5 \cdot 7 & 2.4\end{array}$

$\begin{array}{llll}\text { Centre (Toulouse 2) }(+28) & 75 & 5.5 & 2.3\end{array}$

$\begin{array}{llll}\text { Autre (Dijon)(+19) } & 76 & 5.4 & 2.2\end{array}$

$\begin{array}{llll}\text { Centre (Limoges)(-9) } & 77 & 5.4 & 2.2\end{array}$

Non-Gredeg (Nice)(-13) $\quad 78 \quad 5.3 \quad 2.2$

Autre (Montpellier 1-Inra)(-24) $79 \quad 5.2 \quad 2.1$

Centre (Marne La Vallee)(-37) $\quad 80 \quad 5.1 \quad 2.1$

Centre (Lyon 1)(-12) $\quad 81 \quad 4.9 \quad 2.0$

$\begin{array}{llll}\text { Centre (Rouen) }(+15) & 82 & 4.9 & 2.0\end{array}$

$\begin{array}{llll}\text { Centre (Tours) }(-38) & 83 & 4.9 & 2.0\end{array}$

$\begin{array}{llll}\text { Centre (Paris 12) }(+6) & 84 & 4.7 & 2.0\end{array}$

Centre (La Reunion)(-31) $\quad 85 \quad 4.6 \quad 1.9$

$\begin{array}{llll}\text { Centre (Littoral)(-37) } & 86 & 4.2 & 1.7\end{array}$

$\begin{array}{llll}\text { Centre (Angers) }(-3) & 87 & 4.1 & 1.7\end{array}$

Autre (Grenoble 2-Inra)(-24) $\quad 88 \quad 4.0 \quad 1.7$

Non-Crem (Caen-Rennes 1)(-6) $\quad 89 \quad 3.6 \quad 1.5$

Non-Beta (Nancy 2-Strasb. 1)(o) $90 \quad 3.3 \quad 1.4$

$\begin{array}{llll}\text { Centre (Artois) }(-15) & 91 & 3.2 & 1.3\end{array}$

\begin{tabular}{llll} 
Centre (Paris 5)(-30) & 92 & 3.2 & 1.3 \\
\hline
\end{tabular}

suite page suivante 
suite de la page précédente

\begin{tabular}{|c|c|c|c|c|c|c|c|}
\hline centre (top 10) & rg. & p.c. & nor. & centre (top 30) & rg. & p.c. & nor. \\
\hline Centre (Lyon 1)(-7) & 93 & 4.9 & 0.8 & Centre (Metz)(-14) & 93 & 3.1 & 1.3 \\
\hline Centre (Le Havre) $(-4)$ & 94 & 4.2 & 0.7 & Centre (Le Havre) $(-7)$ & 94 & 2.8 & 1.2 \\
\hline Centre (Artois) $(-12)$ & 95 & 4.0 & 0.7 & Centre (Toulouse 3) $(+5)$ & 95 & 2.8 & 1.1 \\
\hline Centre (Metz)(-10) & 96 & $3 \cdot 9$ & 0.6 & Centre (Brest)(-22) & 96 & 2.5 & 1.0 \\
\hline Centre (Paris 5)(-20) & 97 & $3 \cdot 3$ & 0.6 & Centre (Ant. Guy.)(-21) & 97 & 2.4 & 1.0 \\
\hline Centre (Amiens)(-10) & 98 & 2.9 & 0.5 & Centre (Poitiers) $(-23)$ & 98 & 2.3 & 0.9 \\
\hline Centre (Toulouse 3$)(+1)$ & 99 & 2.8 & 0.5 & Centre (Corte) $(-3)$ & 99 & 1.8 & 0.7 \\
\hline Non-Economix (Paris 10) $(-5)$ & 100 & 2.6 & 0.4 & Non-Economix (Paris 10)(-3) & 100 & 1.8 & 0.8 \\
\hline Centre (Corte) $(-3)$ & 101 & 1.8 & 0.3 & Centre (Bretagne Sud)(-2I) & 101 & 1.5 & 0.6 \\
\hline Centre (Bretagne Sud)(-10) & 102 & 1.5 & 0.2 & Centre (Amiens)(-8) & 102 & 1.4 & 0.6 \\
\hline Centre $($ Lyon 3$)(-2)$ & 103 & 0.9 & 0.2 & Centre (Lyon 3)(-3) & 103 & 0.9 & 0.4 \\
\hline
\end{tabular}

Notes : La colonne "rg." donne le rang, la colonne "tot." donne le score total, "nor." le score normalisé par rapport à celui du premier classé, "p.c." donne le score par chercheur. Entre les premières parenthèses se trouve le nombre de chercheurs, la variation de classement par rapport à Clm est donnée entre les deuxièmes parenthèses. Si cette variation est supérieure ou égale à 3, le nom est typographié en gras italique, si elle est inférieure ou égale à -3 , le nom est typographié en italique, sinon le nom est simplement typographié en gras.

TABLE 31 - Centres, 10 ou 30 productifs, Indice G, T=Dégressif

\begin{tabular}{|c|c|c|c|}
\hline centre (top 10) & rg. & p.c. & nor. \\
\hline Pjse (Pse-Paris 1$)(+1)$ & 1 & 21.3 & 100.0 \\
\hline Gremaq (Tse-Toulouse 1$)(-1)$ & 2 & $19 \cdot 3$ & 90.2 \\
\hline Ces (Pse-Paris 1$)(+2)$ & 3 & 14.6 & 68.3 \\
\hline Greqam (Aix Marseille 2-3)(-1) & 4 & 11.0 & 51.5 \\
\hline Lerna (Tse-Toulouse 1$)(+2)$ & 5 & 10.8 & 50.9 \\
\hline Preg (Ec. Polytechnique)(o) & 6 & 9.9 & 46.2 \\
\hline Non-Grecsta (Crest-Ensae) $(+2)$ & 7 & 9.6 & 45.0 \\
\hline Centre (Iep Paris) $(+4)$ & 8 & 9.2 & $43 \cdot 3$ \\
\hline a (Crest-Ensae)(o) & 9 & 9.2 & 42.9 \\
\hline & 10 & 9.1 & 42.6 \\
\hline 10$)(+2)$ & 11 & 9.0 & 42.4 \\
\hline y 2 -Strasb. 1$)(-1)$ & 12 & 8.3 & 38.8 \\
\hline & 13 & 7.8 & 36.6 \\
\hline & 14 & $7 \cdot 3$ & 34.2 \\
\hline & & 7.1 & 33.4 \\
\hline es 1$)(+1)$ & 16 & 6.9 & 32.3 \\
\hline & 17 & $\cdot 7$ & 31.3 \\
\hline & & 7 & 31.2 \\
\hline & 19 & 6.6 & 31.1 \\
\hline llier 1-Inra)(-5) & 20 & 6.4 & 29.9 \\
\hline Gri & 21 & 6.3 & $29 \cdot 3$ \\
\hline & 22 & 6.1 & 28.6 \\
\hline$(+7)$ & 23 & $5 \cdot 9$ & $27 \cdot 7$ \\
\hline$(+5)$ & 24 & $5 \cdot 7$ & 26.5 \\
\hline & 25 & $5 \cdot 3$ & 25.0 \\
\hline & 26 & 5.2 & $24 \cdot 5$ \\
\hline (e 1-Poly. Lille $)(+17)$ & 27 & 5.1 & 24.0 \\
\hline Ouentin $(+2$ ) & 28 & 5.0 & $23 \cdot 3$ \\
\hline & 29 & & 22.6 \\
\hline & 30 & 4.8 & 22.7 \\
\hline n) $(+63)$ & 31 & $4 \cdot 7$ & 22.1 \\
\hline $2-\operatorname{Inra})(+49)$ & 32 & $4 \cdot 7$ & 22.0 \\
\hline$(-17)$ & 33 & 4.6 & 21.4 \\
\hline & & 4.6 & 21.5 \\
\hline$n r a)(+27)$ & 35 & 4.6 & 21.6 \\
\hline & 36 & $4 \cdot 5$ & 21.1 \\
\hline oly. Lille) $(-14)$ & 37 & $4 \cdot 5$ & 21.3 \\
\hline$(+61)$ & 38 & 4.4 & 20.7 \\
\hline & 39 & & 20.6 \\
\hline 1$)(+31)$ & 40 & $4 \cdot 3$ & 20.1 \\
\hline & 41 & $4 \cdot 3$ & 20.4 \\
\hline Non-Gretha $($ Bordeaux 4$)(+1)$ & 42 & $4 \cdot 3$ & 20.2 \\
\hline
\end{tabular}

\begin{tabular}{|c|c|c|c|}
\hline centre (top 30) & rg. & p.c. & nor. \\
\hline Pjse (Pse-Paris 1$)(+1)$ & 1 & 11.9 & 100.0 \\
\hline Gremaq (Tse-Toulouse 1$)(-1)$ & 2 & 11.2 & 93.9 \\
\hline Ces (Pse-Paris 1$)(0)$ & 3 & 9.8 & 82.4 \\
\hline Centre (Iep Paris) $(+3)$ & 4 & 9.2 & $77 \cdot 4$ \\
\hline Lerna (Tse-Toulouse 1$)(0)$ & 5 & 7.1 & \\
\hline Greqam (Aix Marseille 2-3)(-2) & 6 & 7.0 & 58.8 \\
\hline Greghec $(\mathrm{Hec})(-1)$ & 7 & 6.4 & $53 \cdot 3$ \\
\hline Economix $($ Paris 1o) $(+3)$ & 8 & 5.6 & 47.0 \\
\hline Centre (Cired) $(+12)$ & 9 & $5 \cdot 5$ & 46.3 \\
\hline Preg (Ec. Polytechnique)(o) & 10 & $5 \cdot 5$ & 46.1 \\
\hline Beta (Nancy 2-Strasb. 1)(-1) & 11 & $5 \cdot 3$ & 44.2 \\
\hline Eco. Pub. (Inra Vers-Grig)(+4) & 12 & $5 \cdot 3$ & 44.8 \\
\hline$(-5)$ & 13 & 5.0 & 41.5 \\
\hline Smart (Inra Rennes) $(+2)$ & 14 & 4.9 & 41.5 \\
\hline Non-Grecsta (Crest-Ensae)(-1) & 15 & $4 \cdot 7$ & 39.1 \\
\hline & 16 & 4.5 & 37.6 \\
\hline & 17 & $4 \cdot 3$ & 36.5 \\
\hline Drm (Paris 9) $(+21)$ & 18 & 4.1 & $34 \cdot 5$ \\
\hline Thema (Cergy Pontoise)(-7) & 19 & 4.1 & $34 \cdot 3$ \\
\hline ijon) $(+65)$ & 20 & 4.0 & 33.7 \\
\hline & 21 & 3.8 & 32.2 \\
\hline 1)(+12) & 22 & 3.6 & 30.5 \\
\hline & 23 & $3 \cdot 5$ & 29.0 \\
\hline y) $(+33)$ & 24 & $3 \cdot 5$ & 29.0 \\
\hline & 25 & $3 \cdot 3$ & 27.6 \\
\hline$(+37)$ & 26 & 3.2 & 27.0 \\
\hline g 3$)(-6)$ & 27 & 3.2 & 27.2 \\
\hline 9$)(+1)$ & 28 & 3.2 & 26.7 \\
\hline$u \times 4)(+7)$ & 29 & 3.0 & $25 \cdot 4$ \\
\hline oly. Lille $)(+8)$ & 30 & 3.0 & $25 \cdot 5$ \\
\hline 11$)(+70)$ & 31 & 2.9 & $24 \cdot 3$ \\
\hline 2-Inra)(-1) & 32 & 2.8 & 23.9 \\
\hline ellier $1-\mathrm{Ir}$ & 33 & 2.8 & 23.6 \\
\hline co $($ Aix Marseille 2-3) +5 & 34 & 2.8 & 23.1 \\
\hline$($ Grenoble 2-Inra) $(+45)$ & 35 & 2.7 & 22.4 \\
\hline llier 1-Inra $(+15)$ & 36 & 2.7 & 22.4 \\
\hline $\operatorname{Leg}($ & 37 & 2.6 & 21.6 \\
\hline ielle)(-19) & 38 & 2.5 & 20.8 \\
\hline Lirhe-Eco (Tse-Toulouse 1)(+54) & 39 & 2.5 & 21.1 \\
\hline & 40 & 2.4 & 20.0 \\
\hline$(-8)$ & 41 & 2.4 & 20.4 \\
\hline Centre (Vers. St Quentin) $(+30)$ & 42 & 2.4 & 19.8 \\
\hline
\end{tabular}


suite de la page précédente

\begin{tabular}{|c|c|c|c|c|c|c|c|}
\hline centre (top 10) & rg. & p.c. & nor. & centre (top 30) & rg. & p.c. & nor. \\
\hline Mans $)(-29)$ & 43 & 4.2 & $19 \cdot 5$ & Clerse-Eco (Lille 1-Poly. Lille) $(+12)$ & 43 & 2.4 & 20.0 \\
\hline Autre (Aix Marseille 2-3)(+15) & 44 & 4.1 & $19 \cdot 3$ & Non-Gate (Lyon 2)(+32) & 44 & 2.4 & 19.9 \\
\hline Centre $($ Reims $)(+1)$ & 45 & 4.1 & $19 \cdot 4$ & Centre (Le Mans)(-28) & 45 & 2.3 & $19 \cdot 5$ \\
\hline Lirhe-Eco (Tse-Toulouse 1) $(+43)$ & 46 & 4.1 & 19.1 & Centre (Montpellier 3) $(-3)$ & 46 & 2.3 & 19.5 \\
\hline Centre (Strasbourg 3)(-15) & 47 & 4.0 & 18.9 & Centre (Nantes) $(-22)$ & 47 & 2.3 & $19 \cdot 3$ \\
\hline Gael (Grenoble 2-Inra)(-10) & 48 & 3.9 & 18.3 & Centre $($ Rennes 2$)(+23)$ & 48 & 2.3 & 19.0 \\
\hline Centre $(\mathrm{Pau})(+7)$ & 49 & 3.6 & 16.7 & Ermes (Paris 2)(-17) & 49 & 2.3 & 19.5 \\
\hline Centre (Evry)(-13) & 50 & $3 \cdot 5$ & 16.4 & Non-Cermes (Paris 11) $(+13)$ & 50 & 2.3 & 19.4 \\
\hline Lef $($ Inra Nancy $)(+27)$ & 51 & $3 \cdot 5$ & 16.2 & Centre (Evry)(-16) & 51 & 2.2 & 18.5 \\
\hline Non-Cermes (Paris 11) $(+13)$ & 52 & 3.5 & 16.6 & Centre $(\mathrm{Pau})(+6)$ & 52 & 2.2 & 18.2 \\
\hline ry) $(-26)$ & 53 & $3 \cdot 4$ & $15 \cdot 9$ & Cesaer (Inra Dijon)(-30) & 53 & 2.2 & 18.4 \\
\hline Autre (Lille 1-Poly. Lille)(-22) & 54 & $3 \cdot 4$ & 16.1 & Lepii (Grenoble 2-Inra) $(+41)$ & 54 & 2.2 & 18.3 \\
\hline Centre (Paris 8)(-11) & 55 & $3 \cdot 4$ & 15.9 & Aliss (Inra Ivry)(-29) & 55 & 2.1 & $17 \cdot 5$ \\
\hline Ermes (Paris 2)(-21) & 56 & $3 \cdot 4$ & 16.1 & Autre (Tse-Toulouse 1)(-31) & 56 & 2.1 & 17.8 \\
\hline Lest-Eco (Aix Marseille 2-3)(+36) & 57 & $3 \cdot 3$ & 15.6 & Centre (Lille 3)(-17) & 57 & 2.1 & 17.8 \\
\hline Centre (Paris 12) $(+16)$ & 58 & 3.2 & 14.8 & Non-Cepn (Paris 13) $(+13)$ & 58 & 2.0 & 16.7 \\
\hline Mona-Tsv (Inra Ivry) $(+43)$ & 59 & 3.2 & 15.2 & Autre (Aix Marseille 2-3)(+23) & 59 & 1.9 & $15 \cdot 7$ \\
\hline$e($ Rouen $)(+28)$ & 60 & 3.0 & 14.2 & & 60 & 1.9 & 16.1 \\
\hline$(-10)$ & 61 & 3.0 & 13.9 & $\operatorname{Ivry})(+43)$ & 61 & 1.9 & 16.2 \\
\hline $2-I n r a)(-17)$ & 62 & 2.9 & 13.6 & & 62 & 1.8 & 14.8 \\
\hline lier 1-Inra) $(-15)$ & 63 & 2.8 & 12.9 & & 63 & 1.8 & 15.5 \\
\hline an) $(-16)$ & 64 & 2.8 & 13.1 & $($ Bordeaux 4)(-4) & 64 & 1.8 & 14.8 \\
\hline -Rennes 1$)(+1)$ & 65 & 2.8 & 13.2 & & 65 & 1.7 & 14.0 \\
\hline ier $1-\operatorname{In} r a)(+6)$ & 66 & 2.7 & 12.5 & & 66 & 1.7 & 14.6 \\
\hline 2$)(-28)$ & 67 & 2.6 & 12.4 & tienne)(-7) & 67 & 1.7 & 14.5 \\
\hline n) $(-19)$ & 68 & 2.5 & 11.6 & 1) $(+17)$ & 68 & 1.6 & 13.4 \\
\hline lle) $(-28)$ & 69 & 2.5 & 11.6 & e La Vallee) $(-25)$ & 69 & 1.6 & 13.1 \\
\hline n) $(-27)$ & 70 & 2.4 & 11.3 & $8)(-16)$ & 70 & 1.6 & 13.6 \\
\hline 13$)(+11)$ & 71 & 2.4 & 11.2 & & 71 & 1.6 & 13.6 \\
\hline Cent & 72 & 2.3 & 11.0 & e) $(-3)$ & 72 & 1.6 & 13.4 \\
\hline ier 3$)(-3)$ & 73 & 2.3 & 10.9 & 2-Inra)(-9) & 73 & 1.5 & 12.6 \\
\hline$(+12)$ & 74 & 2.3 & 10.6 & e) $(-33)$ & 74 & 1.5 & 12.5 \\
\hline & 75 & 2.2 & 10.2 & $(+30)$ & 75 & 1.5 & 12.5 \\
\hline$(-20)$ & 76 & 2.2 & 10.1 & y. Lille $)(-35)$ & 76 & 1.4 & 11.7 \\
\hline$)(-20)$ & 77 & 2.1 & 9.7 & (r 1 -Inra) $(-21)$ & 77 & 1.4 & 11.5 \\
\hline cy 2 -Śtrasb. 1) $(+2)$ & 78 & 2.1 & 10.0 & on) $(-22)$ & 78 & 1.4 & 11.4 \\
\hline 6) & 79 & 1.9 & 9.0 & 20) & 79 & 1.4 & 11.9 \\
\hline$(+11)$ & 80 & 1.9 & 8.9 & S 2) $(-31)$ & 80 & 1.4 & 11.6 \\
\hline$(-5)^{\prime}$ & 81 & 1.9 & 9.0 & $(+6)$ & 81 & 1.3 & 10.7 \\
\hline & 82 & 1 . & 8.3 & & 82 & 1.3 & 11.2 \\
\hline & 83 & 1.7 & 8.1 & +9) & 83 & 1.3 & 10.8 \\
\hline$(+13)$ & 84 & 1.7 & 8.1 & $(-17)$ & 84 & 1.2 & 9.9 \\
\hline 1y.) $(-20)$ & 85 & 1.6 & $7 \cdot 4$ & & 85 & 1.2 & 10.1 \\
\hline & 86 & 1.6 & $7 \cdot 4$ & & 86 & 1.0 & 8.5 \\
\hline Vallee) $(-15)$ & 87 & 1.6 & $7 \cdot 7$ & & 87 & 1.0 & 8.4 \\
\hline$(-22)$ & 88 & 1.5 & 7.0 & & 88 & 1.0 & 8.5 \\
\hline$(+15)$ & 89 & 1. & 7.0 & Rennes 1$)(-3)$ & 89 & 1.0 & 8.8 \\
\hline & 90 & 1. & 6.8 & & 90 & 0.9 & $7 \cdot 7$ \\
\hline & 91 & 1.4 & 6.6 & & 91 & o. & $7 \cdot 4$ \\
\hline$)(-4)$ & 92 & 1.3 & 6.2 & 5)(-29) & 92 & 0.9 & 7.8 \\
\hline & 93 & 1.1 & 5.2 & cs) $(-15)$ & 93 & 0.9 & $7 \cdot 4$ \\
\hline & 94 & 1.1 & 5.1 & 2-Strasb. 1)(o) & 94 & 0.9 & $7 \cdot 7$ \\
\hline$(-18)$ & 95 & 1. & 4.6 & & 95 & 0.8 & 6.6 \\
\hline 3$)(+5)$ & 96 & 1. & 4.7 & y.) $(-19)$ & 96 & 0.8 & 6.8 \\
\hline ines) $(-4)$ & 97 & 1. & 4.8 & & 97 & 0.8 & 7.0 \\
\hline & 98 & o. & $4 \cdot 4$ & e Sud)(-15) & 98 & 0.8 & 6.4 \\
\hline & 99 & & $4 \cdot 3$ & $(+1)$ & 99 & 0.8 & 7.0 \\
\hline Sud $)(-8)$ & 100 & 0.8 & 3.6 & $(-24)$ & 100 & 0.7 & 6.3 \\
\hline & 101 & 0.8 & 3.9 & ens) $(-7)$ & 101 & 0.6 & \\
\hline Páric 10)(-1- & 102 & 0.8 & $3 \cdot 5$ & $x$ (Paris 10)(-6) & 102 & 0.5 & \\
\hline Centre $($ Lyon 3$)(-2)$ & 103 & 0.4 & 2.1 & Centre (Lyon 3)(-3) & 103 & 0.4 & 3.8 \\
\hline
\end{tabular}

Notes : La colonne "rg." donne le rang, la colonne "tot." donne le score total, "nor." le score normalisé par rapport à celui du premier classé, "p.c." donne le score par chercheur. Entre les premières parenthèses se trouve le nombre de chercheurs, la variation de classement par rapport à Clm est donnée entre les deuxièmes parenthèses. Si cette variation est supérieure ou égale à 3, le nom est typographié en gras italique, si elle est inférieure ou égale à -3, le nom est typographié en italique, sinon le nom est simplement typographié en gras. 


\section{B.2 Universités}

TABLE 32 - Universités, 10 ou 30 aux plus productifs, Citations totales, T=Dégressif

\begin{tabular}{|c|c|c|c|c|c|c|c|}
\hline université (top 10) & rg. & p.c. & nor. & université (top 30) & rg. & p.c. & nor. \\
\hline Tse-Toulouse $\mathbf{I}(0)$ & 1 & 675.6 & 100.0 & Tse-Toulouse $\mathbf{I}(0)$ & 1 & 279.8 & 100.0 \\
\hline Pse-Paris $1(0)$ & 2 & 468.2 & 69.3 & Pse-Paris $1(0)$ & 2 & 235.4 & 84.1 \\
\hline Crest-Ensae $(+2)$ & 3 & 230.4 & 34.1 & Crest-Ensae(+1) & 3 & 107.7 & 38.5 \\
\hline $\mathrm{Hec}(\mathrm{o})$ & 4 & 158.2 & 23.4 & Iep Paris $(+2)$ & 4 & 101.2 & 36.2 \\
\hline Aix Marseille 2-3(-2) & 5 & 135.1 & 20.0 & $\operatorname{Hec}(\mathrm{o})$ & 5 & 85.2 & 30.5 \\
\hline Iep Paris $(+4)$ & 6 & 101.2 & 15.0 & Aix Marseille 2-3(-3) & 6 & 65.1 & $23 \cdot 3$ \\
\hline Ec. Polytechnique(-1) & 7 & 96.2 & 14.2 & Inra Vers-Grig $(+5)$ & 7 & 43.1 & 15.4 \\
\hline Paris $9(+1)$ & 8 & 85.4 & 12.6 & Paris $9(+6)$ & 8 & 41.7 & 14.9 \\
\hline Paris $10(+2)$ & 9 & 80.7 & 12.0 & Ec. Polytechnique(-2) & 9 & 41.4 & 14.8 \\
\hline Nancy 2 -Strasbourg $\mathbf{I}(-2)$ & 10 & 76.9 & 11.4 & Nancy 2 -Strasbourg $1(-2)$ & 10 & 38.4 & 13.7 \\
\hline Montpellier 1-Inra $(+1)$ & 11 & 73.9 & 10.9 & Paris 10(-2) & 11 & 37.1 & $13 \cdot 3$ \\
\hline Cergy Pontoise(-5) & 12 & 59.6 & 8.8 & Ens Cachan $(-1)$ & 12 & $34 \cdot 3$ & 12.3 \\
\hline Lyon $2(+6)$ & 13 & $59 \cdot 3$ & 8.8 & Cired $(+11)$ & 13 & 33.4 & 11.9 \\
\hline $\operatorname{Dijon}(+24)$ & 14 & 51.8 & $7 \cdot 7$ & Montpellier 1-Inra $(+4)$ & 14 & 29.6 & 10.6 \\
\hline Inra Vers-Grig $(+2)$ & 15 & 51.4 & 7.6 & Inra Rennes $(0)$ & 15 & 28.6 & 10.2 \\
\hline Nice $(+6)$ & 16 & 51.2 & 7.6 & Lyon $2(+4)$ & 16 & $27 \cdot 9$ & 10.0 \\
\hline Bordeaux 4(-1) & 17 & $47 \cdot 3$ & 7.0 & Cergy Pontoise $(-7)$ & 17 & 26.3 & $9 \cdot 4$ \\
\hline Clermont $\mathbf{1}(+8)$ & 18 & 45.2 & 6.7 & Grenoble 2-Inra $(+5)$ & 18 & 24.8 & 8.9 \\
\hline Cired $(+12)$ & 19 & 44.7 & 6.6 & Dijon $(+27)$ & 19 & 23.8 & 8.5 \\
\hline Grenoble 2-Inra $(+1)$ & 20 & 43.3 & 6.4 & Lille 1-Polytech Lille(-7) & 20 & 23.5 & 8.4 \\
\hline Lille 1-Polytech Lille $(-8)$ & 21 & 40.8 & 6.0 & Bordeaux 4(+1) & 21 & 22.2 & 7.9 \\
\hline Paris 11 $(+22)$ & 22 & 40.5 & 6.0 & Strasbourg $3(-2)$ & 22 & 22.0 & $7 \cdot 9$ \\
\hline Paris 13(+6) & 23 & 40.1 & $5 \cdot 9$ & Nice $(+4)$ & 23 & 21.9 & 7.8 \\
\hline $\operatorname{Besancon}(-1)$ & 24 & 38.5 & $5 \cdot 7$ & Caen-Rennes $1(-8)$ & 24 & 19.1 & 6.8 \\
\hline Paris $2(-5)$ & 25 & 35.6 & 5.3 & Lille $2(+16)$ & 25 & 18.0 & 6.4 \\
\hline Ens Cachan $(-2)$ & 26 & $34 \cdot 3$ & 5.1 & Clermont $1(+6)$ & 26 & 17.9 & 6.4 \\
\hline Caen-Rennes $1(-12)$ & 27 & 33.4 & 5.0 & Paris 11 $(+38)$ & 27 & $17 \cdot 5$ & 6.3 \\
\hline Inra Rennes $(-1)$ & 28 & 33.1 & 4.9 & Paris 13 $(+6)$ & 28 & 16.9 & 6.0 \\
\hline Versailles St Quentin $(+12)$ & 29 & 30.9 & 4.6 & Besancon $(0)$ & 29 & 16.8 & 6.0 \\
\hline Inra Ivry $(-5)$ & 30 & 29.2 & $4 \cdot 3$ & Paris 2(-2) & 30 & 15.2 & 5.4 \\
\hline Strasbourg $3(-1)$ & 31 & 28.0 & 4.1 & La Rochelle(-12) & 31 & 14.0 & 5.0 \\
\hline Nantes $(-14)$ & 32 & 26.8 & 4.0 & Chambery $(+20)$ & 32 & 13.8 & 4.9 \\
\hline Lille $2(+13)$ & 33 & $23 \cdot 3$ & $3 \cdot 5$ & Versailles St Quentin(+24) & 33 & $13 \cdot 3$ & 4.8 \\
\hline Orleans $(-6)$ & 34 & 21.0 & 3.1 & Inra Nancy $(+13)$ & 34 & 12.9 & 4.6 \\
\hline Chambery $(+18)$ & 35 & 20.2 & 3.0 & Nantes $(-9)$ & 35 & 12.8 & 4.6 \\
\hline Le Mans(-21) & 36 & 20.2 & 3.0 & $\operatorname{Pau}(+12)$ & 36 & 12.3 & 4.4 \\
\hline $\operatorname{Pau}(+9)$ & 37 & 20.2 & 3.0 & $\operatorname{Inra} \operatorname{Ivry}(-1)$ & 37 & 11.6 & 4.1 \\
\hline Reims $(-2)$ & 38 & 17.8 & 2.6 & Paris $7(+29)$ & 38 & 11.5 & 4.1 \\
\hline Paris 8(-5) & 39 & 16.7 & 2.5 & Inra Dijon $(-15)$ & 39 & 11.4 & 4.1 \\
\hline $\operatorname{Evry}(-8)$ & 40 & 16.2 & 2.4 & Le Mans $(-23)$ & 40 & 11.2 & 4.0 \\
\hline La Rochelle(-8) & 41 & 14.0 & 2.1 & $\operatorname{Evry}(-10)$ & 41 & $9 \cdot 4$ & $3 \cdot 4$ \\
\hline St Etienne(-2) & 42 & 13.7 & 2.0 & Lille $3(-9)$ & 42 & 8.0 & 2.9 \\
\hline Inra Nancy $(+16)$ & 43 & 12.9 & 1.9 & Orleans $(-3)$ & 43 & 7.8 & 2.8 \\
\hline Inra Dijon(-9) & 44 & 12.6 & 1.9 & Rennes 2(+11) & 44 & $7 \cdot 7$ & 2.8 \\
\hline Paris 12(+13) & $\begin{array}{l}44 \\
45\end{array}$ & 12.6 & 1.9 & Montpellier $3(-8)$ & $\begin{array}{l}44 \\
45\end{array}$ & $7 \cdot 4$ & 2.7 \\
\hline Paris $7(+25)$ & 46 & 11.5 & 1.7 & Perpignan(-15) & 46 & $7 \cdot 4$ & 2.7 \\
\hline Rouen $(+19)$ & 47 & 10.4 & 1.5 & Paris $8(-3)$ & 47 & 6.9 & 2.5 \\
\hline $\operatorname{Limoges}(-2)$ & 48 & 9.6 & 1.4 & $\operatorname{Reims}(+5)$ & 48 & 6.6 & 2.4 \\
\hline Lille $3(-7)$ & 49 & 8.8 & 1.3 & St Etienne $(+1)$ & 49 & 6.6 & 2.4 \\
\hline La Reunion(-11) & 50 & 8.6 & 1.3 & Valenciennes $(+12)$ & 50 & 6.4 & 2.3 \\
\hline Perpignan $(-14)$ & 51 & 8.5 & 1.3 & Mulhouse $(-16)$ & 51 & 6.2 & 2.2 \\
\hline Rennes $2(+11)$ & 52 & $7 \cdot 7$ & 1.1 & $\operatorname{Cnam}(-9)$ & 52 & 6.1 & 2.2 \\
\hline Montpellier 3(+1) & 53 & $7 \cdot 4$ & 1.1 & Toulon $(-3)$ & 53 & $5 \cdot 7$ & 2.1 \\
\hline Angers $(+7)$ & 54 & $7 \cdot 3$ & 1.1 & Toulouse $2(+21)$ & 54 & $5 \cdot 5$ & 2.0 \\
\hline Valenciennes $(+14)$ & 55 & 6.4 & 1.0 & $\operatorname{Limoges}(-1)$ & 55 & 5.4 & 1.9 \\
\hline Toulon $(+2)$ & 56 & 6.3 & 0.9 & Marne La Vallee $(-18)$ & 56 & 5.1 & 1.8 \\
\hline Mulhouse(-6) & 57 & 6.2 & 0.9 & Lyon $\mathbf{1}(-1)$ & 57 & 4.9 & 1.8 \\
\hline $\operatorname{Cnam}(-2)$ & 58 & 6.1 & 0.9 & Rouen $(+14)$ & 58 & 4.9 & 1.7 \\
\hline Tours(-11) & 59 & 5.9 & 0.9 & Tours $(-19)$ & 59 & 4.9 & 1.8 \\
\hline Poitiers $(-17)$ & 60 & 5.8 & 0.9 & Paris 12(+9) & 60 & 4.7 & 1.7 \\
\hline Toulouse $2(+14)$ & 61 & 5.5 & 0.8 & La Reunion $(-16)$ & 61 & $\begin{array}{l}4 \cdot 7 \\
4.6\end{array}$ & 1.6 \\
\hline Littoral(-14) & 62 & 5.4 & 0.8 & Littoral $(-20)$ & 62 & 4.2 & 1.5 \\
\hline Marne La Vallee(-8) & 63 & $5 \cdot 3$ & 0.8 & Angers (+3) & 63 & 4.1 & 1.5 \\
\hline \multirow[t]{2}{*}{$\operatorname{Brest}(-13)$} & 64 & 5.0 & 0.7 & Artois $(-4)$ & 64 & 3.2 & 1.1 \\
\hline & & & & \multicolumn{4}{|c|}{ suite page suivante } \\
\hline
\end{tabular}


suite de la page précédente

\begin{tabular}{|c|c|c|c|c|c|c|c|}
\hline université (top 10) & rg. & p.c. & nor. & université (top 30) & rg. & p.c. & nor. \\
\hline Antilles Guyane(-15) & 65 & 4.9 & 0.7 & Paris $5(-14)$ & 65 & 3.2 & 1.1 \\
\hline Lyon $1(-1)$ & 66 & 4.9 & 0.7 & $\operatorname{Metz}(-3)$ & 66 & 3.1 & 1.1 \\
\hline Le Havre $(+1)$ & 67 & 4.2 & 0.6 & Le Havre(o) & 67 & 2.8 & 1.0 \\
\hline Artois $(-6)$ & 68 & 4.0 & 0.6 & Toulouse $3(+6)$ & 68 & 2.8 & 1.0 \\
\hline $\operatorname{Metz}(-4)$ & 69 & 3.9 & 0.6 & $\operatorname{Brest}(-11)$ & 69 & 2.5 & 0.9 \\
\hline Paris $5(-11)$ & 70 & 3.3 & 0.5 & Antilles Guyane $(-10)$ & 70 & 2.4 & 0.8 \\
\hline Amiens(-5) & 71 & 2.9 & 0.4 & Poitiers $(-12)$ & 71 & 2.3 & 0.8 \\
\hline Toulouse $3(+1)$ & 72 & 2.8 & 0.4 & Corte $(-1)$ & 72 & 1.8 & 0.6 \\
\hline Corte $(-1)$ & 73 & 1.8 & 0.3 & Bretagne Sud(-9) & 73 & 1.5 & 0.5 \\
\hline Bretagne Sud(-4) & 74 & 1.5 & 0.2 & Amiens(-4) & 74 & 1.4 & 0.5 \\
\hline Lyon $3(-1)$ & $\begin{array}{l}14 \\
75\end{array}$ & 0.9 & 0.1 & Lyon $3(-1)$ & 75 & 0.9 & 0.3 \\
\hline
\end{tabular}

Notes : La colonne "rg." donne le rang, la colonne "tot." donne le score total, "nor." le score normalisé par rapport à celui du premier classé, "p.c." donne le score par chercheur. Entre les premières parenthèses se trouve le nombre de chercheurs, la variation de classement par rapport à $\mathrm{Clm}$ est donnée entre les deuxièmes parenthèses. Si cette variation est supérieure ou égale à 3, le nom est typographié en gras italique, si elle est inférieure ou égale à -3 , le nom est typographié en italique, sinon le nom est simplement typographié en gras.

TABLE 33 - Universités, 10 ou 30 plus productifs, Indice G, T=Dégressif

\begin{tabular}{|c|c|c|c|}
\hline université (top 10) & rg. & p.c. & nor. \\
\hline Pse-Paris $\mathbf{I}(+\mathbb{1})$ & 1 & 23.6 & 100.0 \\
\hline Tse-Toulouse $1(-1)$ & 2 & 21.5 & 91.1 \\
\hline Crest-Ensae $(+2)$ & 3 & 12.3 & 52.2 \\
\hline Aix Marseille 2-3(-1) & 4 & 11.1 & 47.0 \\
\hline Ec. Polytechnique $(+1)$ & 5 & 9.9 & 41.7 \\
\hline Iep Paris $(+4)$ & 6 & 9.2 & 39.0 \\
\hline $\operatorname{Hec}(-3)$ & 7 & 9.1 & 38.4 \\
\hline Paris 10 $(+4)$ & 8 & 9.1 & 38.4 \\
\hline Nancy 2-Strasbourg $1(-1)$ & 9 & 8.7 & 36.6 \\
\hline Lyon $2(+9)$ & 10 & 8.1 & 34.1 \\
\hline Cergy Pontoise $(-4)$ & 11 & 7.8 & 33.1 \\
\hline $\operatorname{Nice}(+10)$ & 12 & $7 \cdot 7$ & 32.4 \\
\hline Paris 9 $(-4)$ & 13 & $7 \cdot 4$ & 31.2 \\
\hline Bordeaux 4(+2) & 14 & $7 \cdot 3$ & 31.0 \\
\hline Cired $(+16)$ & 15 & 7.1 & 30.2 \\
\hline Caen-Rennes $\mathbf{1}(-1)$ & 16 & 7.0 & 29.8 \\
\hline Grenoble 2-Inra(+5) & 17 & 7.0 & $29 \cdot 5$ \\
\hline Paris 13(+13) & 18 & 7.0 & 29.4 \\
\hline $\operatorname{Dijon}(+19)$ & 19 & 6.9 & 29.1 \\
\hline Montpellier 1-Inra(-7) & 20 & 6.9 & 29.1 \\
\hline Inra Vers-Grig(-4) & 21 & 6.3 & 26.4 \\
\hline Lille 1-Polytech Lille(-8) & 22 & 6.3 & 26.6 \\
\hline Clermont 1(+3) & 23 & 6.1 & 25.8 \\
\hline Paris 11(+20) & 24 & 5.8 & 24.4 \\
\hline Inra Rennes(+2) & 25 & $5 \cdot 7$ & 23.9 \\
\hline $\operatorname{Inra} \operatorname{Ivry}(-1)$ & 26 & 5.0 & 21.2 \\
\hline Versailles St Quentin(+15) & 27 & 5.0 & 21.0 \\
\hline Besancon $(-5)$ & 28 & 4.8 & 20.4 \\
\hline Chambery $(+24)$ & 29 & 4.6 & 19.4 \\
\hline Nantes $(-12)$ & 30 & $4 \cdot 5$ & 19.1 \\
\hline Orleans(-3) & 31 & 4.4 & 18.7 \\
\hline Ens Cachan (-8) & 32 & $4 \cdot 3$ & 18.4 \\
\hline Le Mans(-19) & 33 & 4.2 & 17.6 \\
\hline $\operatorname{Reims}(+2)$ & 34 & 4.1 & $17 \cdot 5$ \\
\hline Paris $2(-15)$ & 35 & 4.0 & 16.7 \\
\hline Strasbourg $3(-5)$ & 36 & 4.0 & 17.1 \\
\hline $\operatorname{Pau}(+7)$ & & 3.6 & 15.1 \\
\hline $\operatorname{Evry}(-6)$ & 38 & $3 \cdot 5$ & 14.8 \\
\hline Inra Nancy $(+21)$ & 39 & $3 \cdot 5$ & 14.7 \\
\hline Paris $8(-6)$ & 40 & 3.4 & 14.4 \\
\hline Paris 12(+16) & 41 & 3.2 & 13.4 \\
\hline Rouen $(+24)$ & 42 & 3.0 & 12.8 \\
\hline
\end{tabular}

\begin{tabular}{|c|c|c|c|}
\hline université (top 30) & rg. & p.c. & nor. \\
\hline Pse-Paris $\mathbf{1}(+\mathbf{1})$ & 1 & $15 \cdot 3$ & 100.0 \\
\hline Tse-Toulouse $\mathbf{1}(-1)$ & 2 & 13.5 & 88.3 \\
\hline Iep Paris $(+3)$ & 3 & 9.2 & 60.3 \\
\hline Crest-Ensae(o) & 4 & $7 \cdot 7$ & 50.3 \\
\hline Aix Marseille 2-3(-2) & $\begin{array}{l}4 \\
5\end{array}$ & 7.6 & $\begin{array}{l}50.3 \\
49.4\end{array}$ \\
\hline $\mathrm{Hec}(-1)$ & 6 & 6.4 & 41.5 \\
\hline Paris $10(+2)$ & 7 & 5.7 & $37 \cdot 3$ \\
\hline Cired $(+16)$ & 8 & $5 \cdot 5$ & 36.1 \\
\hline Ec. Polytechnique(-1) & 9 & $5 \cdot 5$ & 35.9 \\
\hline Nancy 2-Strasbourg $1(0)$ & 10 & 5.5 & 35.8 \\
\hline Inra Vers-Grig $(+1)$ & 11 & $5 \cdot 3$ & 34.9 \\
\hline Paris $9(+2)$ & 12 & 5.2 & 34.2 \\
\hline Inra Rennes $(+2)$ & 13 & 4.9 & 32.3 \\
\hline Caen-Rennes $\mathbf{I}(+2)$ & 14 & 4.8 & 31.6 \\
\hline Grenoble 2-Inra $(+8)$ & 15 & 4.7 & 30.5 \\
\hline Lyon $2(+5)$ & 16 & 4.7 & 30.8 \\
\hline Lille 1-Polytech Lille $(-4)$ & 17 & 4.5 & 29.6 \\
\hline Ens Cachan $(-7)$ & 18 & 4.3 & 28.4 \\
\hline Nice $(+8)$ & 19 & 4.2 & $27 \cdot 4$ \\
\hline Bordeaux 4(+2) & 20 & 4.1 & 26.9 \\
\hline Cergy Pontoise $(-10)$ & 21 & 4.1 & 26.8 \\
\hline $\operatorname{Dijon}(+24)$ & 22 & 4.0 & 26.4 \\
\hline Montpellier 1-Inra(-4) & 23 & 4.0 & 26.4 \\
\hline Paris 13(+10) & 24 & 3.9 & 25.2 \\
\hline Inra Nancy $(+22)$ & 25 & 3.5 & 22.6 \\
\hline Chambery $(+26)$ & 26 & 3.2 & 21.0 \\
\hline ont $1(+6)$ & 27 & 3.2 & 20.6 \\
\hline $\mathbf{1}(+39)$ & 28 & 3.2 & 20.6 \\
\hline Strasbourg 3(-6) & 29 & 3.2 & 21.2 \\
\hline Inra Ivry $(+6)$ & 30 & 2.5 & 16.1 \\
\hline La Rochelle $(-11)$ & 31 & 2.5 & 16.2 \\
\hline Besancon $(-3)$ & 32 & 2.4 & 15.6 \\
\hline Paris 2(-4) & 33 & 2.4 & 15.5 \\
\hline Perpignan $(-2)$ & 34 & 2.4 & 15.9 \\
\hline Versailles St Quentin $(+25)$ & 35 & 2.4 & 15.4 \\
\hline Le Mans(-19) & 36 & 2.3 & 15.2 \\
\hline Montpellier $3(+1)$ & 37 & 2.3 & 15.2 \\
\hline Nantes $(-10)$ & 38 & 2.3 & 15.0 \\
\hline Rennes 2(+19) & 39 & 2.3 & 14.8 \\
\hline Evry $(-9)$ & 40 & 2.2 & 14.4 \\
\hline Inra Dijon(-16) & 41 & 2.2 & 14.4 \\
\hline $\operatorname{Pau}(+8)$ & 42 & 2.2 & 14.2 \\
\hline
\end{tabular}


suite de la page précédente

\begin{tabular}{|c|c|c|c|c|c|c|c|}
\hline université (top 10) & rg. & p.c. & nor. & université (top 30) & rg. & p.c. & nor. \\
\hline St Etienne(-2) & 43 & 3.0 & 12.5 & Lille $3(-10)$ & 43 & 2.1 & 13.9 \\
\hline Perpignan $(-7)$ & 44 & 2.8 & 11.8 & $\operatorname{Cnam}(-1)$ & 44 & 1.9 & 12.6 \\
\hline La Reunion $(-6)$ & 45 & 2.5 & 10.5 & Orleans $(-4)$ & 45 & 1.9 & 12.1 \\
\hline La Rochelle $(-12)$ & 46 & 2.5 & 10.5 & $\operatorname{Reims}(+7)$ & 46 & 1.8 & 11.5 \\
\hline Inra Dijon(-12) & 47 & 2.4 & 10.2 & Lille $2(-6)$ & 47 & 1.7 & 10.9 \\
\hline Lille $3(-6)$ & 48 & 2.3 & 9.9 & Paris $7(+20)$ & 48 & 1.7 & 11.3 \\
\hline Montpellier $3(+6)$ & 49 & 2.3 & 9.8 & St Etienne $(+2)$ & 49 & 1.7 & 11.3 \\
\hline Rennes $2(+15)$ & 50 & 2.3 & 9.6 & Marne La Vallee $(-12)$ & 50 & 1.6 & 10.2 \\
\hline Lille $2(-5)$ & 51 & 2.2 & 9.2 & Paris $8(-6)$ & 51 & 1.6 & 10.6 \\
\hline Poitiers $(-8)$ & 52 & 2.2 & 9.1 & Toulon $(\mathrm{o})$ & 52 & 1.6 & 10.6 \\
\hline Limoges $(-7)$ & 53 & 2.1 & 8.8 & Mulhouse(-18) & 53 & 1.5 & $9 \cdot 7$ \\
\hline $\operatorname{Cnam}(+2)$ & 54 & 1.9 & 8.1 & Toulouse 2(+22) & 54 & 1.5 & 9.8 \\
\hline Le Havre (+14) & 55 & 1.9 & 8.0 & La Reunion(-10) & 55 & 1.4 & 8.9 \\
\hline Toulon $(+2)$ & 56 & 1.8 & $7 \cdot 5$ & Rouen $(+16)$ & 56 & 1.4 & $9 \cdot 3$ \\
\hline Littoral $(-9)$ & 57 & 1.7 & $7 \cdot 3$ & Le Havre $(+10)$ & 57 & 1.3 & 8.4 \\
\hline Paris $7(+14)$ & 58 & 1.7 & $7 \cdot 3$ & Littoral $(-15)$ & 58 & 1.3 & 8.7 \\
\hline Antilles Guyane(-9) & 59 & 1.6 & 6.7 & Paris 12(+12) & 59 & 1.3 & 8.4 \\
\hline Brest $(-8)$ & 60 & 1.6 & 6.7 & Limoges(-6) & 60 & 1.2 & $7 \cdot 7$ \\
\hline Marne La Vallee(-4) & 61 & 1.6 & 6.9 & Tours $(-22)$ & 61 & 1.2 & $7 \cdot 9$ \\
\hline Mulhouse(-11) & 62 & 1.5 & 6.3 & Toulouse $3(+11)$ & 62 & 1.0 & 6.6 \\
\hline Toulouse $2(+13)$ & 63 & 1.5 & 6.3 & Valenciennes(o) & 63 & 1.0 & 6.6 \\
\hline Tours $(-14)$ & 64 & 1.5 & 6.1 & Lyon $1(-8)$ & 64 & 0.9 & 6.0 \\
\hline Angers(-4) & 65 & 1.4 & $5 \cdot 9$ & $\operatorname{Metz}(-1)$ & 65 & 0.9 & $5 \cdot 7$ \\
\hline Amiens(o) & 66 & 1.3 & 5.6 & Paris $5(-14)$ & 66 & 0.9 & 6.1 \\
\hline $\operatorname{Metz}(-2)$ & 67 & 1.1 & 4.6 & Poitiers $(-5)$ & 67 & 0.9 & 5.8 \\
\hline Paris 5(-9) & 68 & 1.0 & 4.1 & Angers $(-2)$ & 68 & 0.8 & 5.1 \\
\hline Toulouse $3(+5)$ & 69 & 1.0 & 4.3 & Antilles Guyane $(-8)$ & 69 & 0.8 & $5 \cdot 3$ \\
\hline Valenciennes $(+1)$ & 70 & 1.0 & $4 \cdot 3$ & Brest $(-10)$ & 70 & 0.8 & $5 \cdot 4$ \\
\hline Artois $(-9)$ & 71 & 0.9 & 4.0 & Bretagne Sud $(-4)$ & 71 & 0.8 & 5.0 \\
\hline Lyon $1(-7)$ & 72 & 0.9 & $3 \cdot 9$ & Corte $(+3)$ & 72 & 0.8 & $5 \cdot 4$ \\
\hline Bretagne Sud(-3) & 73 & 0.8 & 3.3 & Artois(-13) & 73 & 0.7 & 4.9 \\
\hline Corte $(-1)$ & 74 & 0.8 & $\begin{array}{l}3.3 \\
3.5\end{array}$ & Amiens(-4) & 74 & 0.6 & $\begin{array}{l}4.9 \\
4.1\end{array}$ \\
\hline Lyon $3(-1)$ & 75 & 0.4 & 1.9 & Lyon $3(-1)$ & 75 & 0.4 & 2.9 \\
\hline
\end{tabular}

Notes : La colonne "rg." donne le rang, la colonne "tot." donne le score total, "nor." le score normalisé par rapport à celui du premier classé, "p.c." donne le score par chercheur. Entre les premières parenthèses se trouve le nombre de chercheurs, la variation de classement par rapport à $\mathrm{Clm}$ est donnée entre les deuxièmes parenthèses. Si cette variation est supérieure ou égale à 3, le nom est typographié en gras italique, si elle est inférieure ou égale à -3 , le nom est typographié en italique, sinon le nom est simplement typographié en gras. 